\title{
A quantitative characterisation of functions with low Aviles Giga energy on convex domains
}

\author{
ANDREW LORENT
}

\begin{abstract}
Given a connected Lipschitz domain $\Omega$ we let $\Lambda(\Omega)$ be the set of functions in $W^{2,2}(\Omega)$ with $u=0$ on $\partial \Omega$ and whose gradient (in the sense of trace) satisfies $\nabla u(x) \cdot \eta_{x}=1$, where $\eta_{x}$ is the inward pointing unit normal to $\partial \Omega$ at $x$. The functional $I_{\epsilon}(u)=\left.\left.\frac{1}{2} \int_{\Omega} \epsilon^{-1}|1-| \nabla u\right|^{2}\right|^{2}+\epsilon\left|\nabla^{2} u\right|^{2} d z$, minimised over $\Lambda(\Omega)$, serves as a model in connection with problems in liquid crystals and thin film blisters. It is also the most natural higher order generalisation of the Modica and Mortola functional. In [16] Jabin, Otto and Perthame characterised a class of functions which includes all limits of sequences $u_{n} \in \Lambda(\Omega)$ with $I_{\epsilon_{n}}\left(u_{n}\right) \rightarrow 0$ as $\epsilon_{n} \rightarrow 0$. A corollary to their work is that if there exists such a sequence $\left(u_{n}\right)$ for a bounded domain $\Omega$, then $\Omega$ must be a ball and (up to change of sign) $u:=\lim _{n \rightarrow \infty} u_{n}$ is equal $\operatorname{dist}(\cdot, \partial \Omega)$. We prove a quantitative generalisation of this corollary for the class of bounded convex sets. Namely we show that there exists a positive constant $\gamma_{1}$ such that, if $\Omega$ is a convex set of diameter 2 and
\end{abstract} $u \in \Lambda(\Omega)$ with $I_{\epsilon}(u)=\beta$, then $\left|B_{1}(x) \triangle \Omega\right| \leq c \beta^{\gamma_{1}}$ for some $x$ and

$$
\int_{\Omega}\left|\nabla u(z)+\frac{z-x}{|z-x|}\right|^{2} d z \leq c \beta^{\gamma_{1}} .
$$

A corollary of this result is that there exists a positive constant $\gamma_{2}<\gamma_{1}$ such that if $\Omega$ is convex with diameter 2 and $C^{2}$ boundary with curvature bounded by $\epsilon^{-\frac{1}{2}}$, then for any minimiser $v$ of $I_{\epsilon}$ over $\Lambda(\Omega)$ we have

$$
\|v-\zeta\|_{W^{1,2}(\Omega)} \leq c\left(\epsilon+\inf _{y}\left|\Omega \triangle B_{1}(y)\right|\right)^{\gamma_{2}},
$$

where $\zeta(z)=\operatorname{dist}(z, \partial \Omega)$. Neither of the constants $\gamma_{1}$ or $\gamma_{2}$ are optimal.

Mathematics Subject Classification (2010): 49N99 (primary).

\section{Introduction}

We consider the following functional

$$
I_{\epsilon}(u)=\left.\left.\frac{1}{2} \int_{\Omega} \epsilon^{-1}|1-| \nabla u\right|^{2}\right|^{2}+\epsilon\left|\nabla^{2} u\right|^{2} d z
$$

the study of which arises from a number of sources, one of the earliest and most important of which is the article by Aviles and Giga [7]. We will refer to the quantity

Received February 1, 2009; accepted in revised form February 20, 2012. 
$I_{\epsilon}(u)$ as the Aviles-Giga energy of the function $u$. The functional $I_{\epsilon}$ is usually minimised over the space of functions $u \in W^{2,2}(\Omega)$ where $u(x)=0$ and $\nabla u(x)$. $\eta_{x}=1$ on $\partial \Omega$ (in the sense of trace) where $\eta_{x}$ is the inward pointing unit normal, we will denote this space of functions by $\Lambda(\Omega)$.

Aviles and Giga raised the problem of the study of the limiting behavior of $I_{\epsilon}$ as $\epsilon \rightarrow 0$ in connection with the theory of smectic liquid crystals [7]. In [14] Gioia and Ortiz studied $I_{\epsilon}$ as a model for thin film blisters. Jin and Kohn [17] introduced the by now classic method of estimating the energy by 'divergence of vector fields'. A related functional arising from micromagnetics was studied by Rivière and Serfaty [24]. In this case the functional acts on vector fields $m$ (in two dimensions) satisfying $|m|=1$ in $\Omega$, and the functional is given by $M_{\epsilon}(m)=$ $\epsilon \int_{\Omega}|\nabla m|^{2}+\epsilon^{-1} \int_{\mathbb{R}^{2}}\left|\nabla^{-1} \operatorname{div} \tilde{m}\right|^{2}$, where $\tilde{m}$ is the vector field $m$ extended trivially by 0 outside $\Omega$. For the Aviles-Giga functional we minimise over curl free vector fields and the functional forces the norm of the vector field to be close to 1 with weighting $\epsilon^{-1}$ while constraining an $\epsilon$ multiple of the $L^{2}$ norm (squared) of the gradient. On the other hand the micromagnetics functional is minimised over vector fields whose norm is taken to be 1 from the outset, and the functional forces the vector field to be divergence free with weighting $\epsilon^{-1}{ }^{1}$, while again constraining an $\epsilon$ multiple of the $L^{2}$ norm (squared) of the gradient. Functional $M_{\epsilon}$ is much more rigid, and very much stronger results are known for it than for $I_{\epsilon}$, see $[1,6,24]$ and [5].

Roughly speaking: the conjecture is that as $\epsilon \rightarrow 0$ the energy of minimisers of $I_{\epsilon}$ will converge to a collection of curves on which the gradient of the minimisers makes a jump of order $O(1)$ perpendicularly across the curve. This has already been proved for functional $M_{\epsilon}$ [24]. A way to think about this is the following: given a connected Lipschitz domain $\Omega$ let $w$ be the distance from $\partial \Omega$ and let $v_{\epsilon}$ be $w$ convolved by a convolution kernel of diameter $\epsilon$. The regions where $\left|\nabla v_{\epsilon}\right| \not 1$ will be exactly the $\epsilon$ neighborhoods of the curves on which $\nabla w$ has a jump discontinuity. If $\Omega$ is a ball $\nabla w$ will have a discontinuity only at one point. In all other cases there will be non trivial curves of singularities, and for the specific function $v_{\epsilon}$ it is exactly in an $\epsilon$ neighborhood of these curves that the energy will concentrate. The conjecture is that what we can observe directly for $v_{\epsilon}$ will hold true for the minimisers of $I_{\epsilon}$.

The most natural way to study these questions is within the framework of $\Gamma$-convergence. One of the earliest successes of $\Gamma$-convergence was the characterisation of the $\Gamma$-limit of the so called Modica-Mortola functional $A_{\epsilon}(w)=$ $\int_{\Omega} \epsilon|\nabla w|^{2}+\left.\left.\epsilon^{-1}|1-| w\right|^{2}\right|^{2}$ which is minimised over scalar functions $w$ satisfying an integral condition of the form $\int_{\Omega} w d x=0$. It was shown by Modica and Mortola [21] (confirming a conjecture of De Giorgi) that the $\Gamma$-limit of $A_{\epsilon}$ is a constant multiple of the $H^{n-1}$ measure of the jump set $J_{w}$ minimised over the space of functions $w \in\left\{v \in B V: v \in\{1,-1\}\right.$ a.e. and $\left.\int v d x=0\right\}$. Given the elementary

${ }^{1}$ The term $\int_{R^{2}}\left|\nabla^{-1} \operatorname{divm}\right|^{2}$ is the $L^{2}$ norm of the Hodge projection onto curl free vector fields. 
inequality

$$
\epsilon|\nabla w|^{2}+\left.\left.\epsilon^{-1}|1-| w\right|^{2}\right|^{2} \geq\left.|\nabla w||1-| w\right|^{2} \mid,
$$

we have that for any sequence $\left(w_{n}\right)$ of equibounded $A_{\epsilon_{n}}$ energy (for some subsequence $\epsilon_{n} \rightarrow 0$ ) has a uniform $L^{1}$ control of $\nabla\left(w_{n}-\frac{w_{n}^{3}}{3}\right)$ and the measure we obtain as the limit of this $L^{1}$ sequence of gradients will naturally be supported on the jump set of the limiting function. In some sense the nature of the $\Gamma$-limit of $A_{\epsilon}$ could be anticipated from (1.1).

Functional $I_{\epsilon}$ is the most natural higher order generalisation of $A_{\epsilon}$. In the case of $I_{\epsilon}$ the conjectured $\Gamma$-limit is surprising. This is part of the reason that functional $I_{\epsilon}$ has received so much attention. The first works on identifying the $\Gamma$-limit are by Aviles and Giga [7] and Jin and Kohn [17]. Later these ideas were developed by Ambrosio, De Lellis and Mantegazza [2]. Roughly speaking, the limiting function space is conjectured to have a structure similar to the space of functions whose gradient is $B V$ and the limiting energy is conjectured to have the form $\int_{J_{\nabla u}}\left|\nabla u^{+}-\nabla u^{-}\right|^{3} d H^{1}$. Much progress has been made on this conjecture. In particular equi-coercivity of $I_{\epsilon}$ has been shown independently in [2] and in the work of DeSimone, Kohn, Muller and Otto [11]. A proposed limiting function space $A G(\Omega)$ and limiting functional $I$ as been suggested in [2], and it was shown that all limits of sequences of functions $\left(u_{n}\right)$ with $\sup _{n} I_{\epsilon_{n}}\left(u_{n}\right)<\infty$ are such that $u_{n} \stackrel{W^{1,3}}{\rightarrow} u \in A G(\Omega)$ and $\lim \inf I_{\epsilon_{n}}\left(\nabla u_{n}\right) \geq I(u)$. The compactness proofs provided by [11] and [2] are different but share some common ideas. The proof by [11] identifies the set of all smooth functions $\Phi: \mathbb{R}^{2} \rightarrow \mathbb{R}^{2}$ for which there exists a smooth $\Psi: \mathbb{R}^{2} \rightarrow \mathbb{R}^{2}$ such that

$$
\int|\operatorname{div}[\Phi(\nabla u)]| \leq c \int\left|\Psi(\nabla u) \cdot \nabla\left(1-|\nabla u|^{2}\right)\right| \text { for any } C^{2} \text { function } u \text {. }
$$

Influenced by ideas of Tartar and Murat on compensated compactness [25] [22] the authors were able to prove that this set of $\Phi$ is sufficiently rich so as to force $\nabla u_{n}$ to converge strongly. In [7] the authors, building on work of Jin and Kohn [17], found two third order polynomial vector fields $\Sigma_{1}: \mathbb{R}^{2} \rightarrow \mathbb{R}^{2}$ and $\Sigma_{2}: \mathbb{R}^{2} \rightarrow \mathbb{R}^{2}$ such that

$\int\left|\operatorname{div}\left[\Sigma_{i}(\nabla u)\right]\right| \leq\left. c \int\left|\nabla^{2} u\right||1-| \nabla u\right|^{2} \mid$ for any $C^{2}$ function $u$, for $i=1,2$.

Using some elementary and surprising identities satisfied by $\Sigma_{1}(\nabla u), \Sigma_{2}(\nabla u)$, a different approach to compactness was found. Rather naturally considering (1.3). The function space $A G(\Omega)$ proposed by [2] is given by the set of functions $v$ for which $\operatorname{div}\left(\Sigma_{i}(\nabla v)\right)$ forms a Radon measure for $i=1,2$ and the limiting energy functional $I(v)$ is given by the total absolute value of this measure on $\Omega$.

Given vector field $w$ let $\chi(\xi, w):=\mathbb{1}_{\{\xi \cdot w>0\}}$, Jabin and Perthame [15] showed that gradients of sequences of bounded Aviles-Giga energy (in fact their method 
extends to more general functionals) are compact and the limit $\nabla u$ satisfies a kinetic equation of the form $\xi \cdot \nabla_{x} \chi(\xi, R(\nabla u))=q$ where $q$ is the distribution derivative with respect to $\xi$ of some measure on $\mathbb{R}_{\xi}^{2} \times \mathbb{R}_{x}^{2}$ and $R$ is the rotation given by $R(x, y)=(-y, x)$. By application of kinetic averaging lemmas [12] this leads to some regularity: $\nabla u \in W^{s, q}$ for all $0 \leq s<\frac{1}{5}, q<\frac{5}{3}$, and using the kinetic equation a different proof of compactness was found. The kinetic equation deduced by [15] was motivated by the characterisation of the set of $\Phi$ satisfying (1.2) given in [11]: defining $\tilde{\Phi}(z)=|z|^{2} e$ for $z \cdot e>0$ and 0 otherwise it was shown that a sequence $\Phi_{n}$ satisfying (1.2) could be found that approximates $\tilde{\Phi}$ pointwise. Using the kinetic equation deduced in [15], Jabin, Otto and Perthame [16] were able to characterise zero energy limits (and the domains that allow them) for $I_{\epsilon}$. In fact their result is stronger: they showed that if a divergence free vector field $m$ satisfies the kinetic equation $\xi \cdot \nabla \chi(m, \xi)=0,|m(x)|=1$ a.e. in $\Omega$ and $m(x) \cdot \eta_{x}=0$ on $\partial \Omega$, then either $\Omega$ is a strip and $m$ is a constant or $\Omega=B_{r}(x)$ for some $r>0$, $x \in \mathbb{R}^{2}$ and $m(z)=\left(\frac{z-x}{|z-x|}\right)^{\perp}$ or $m(z)=-\left(\frac{z-x}{|z-x|}\right)^{\perp}$. An analogous result for zero energy limits of $M_{\epsilon}$ is stated in [18] and is a consequence of the main theorem of [5].

As a corollary: given a sequence $u_{n} \in \Lambda(\Omega)$ and $\epsilon_{n} \rightarrow 0$ such that $I_{\epsilon_{n}}\left(u_{n}\right) \rightarrow$ 0 as $n \rightarrow \infty$ and letting $u$ be the limit of this sequence, then the vector field $R(\nabla u)$ satisfies the hypothesis stated and hence we have (up to a sign) a complete description of $\nabla u$.

The main theorem of this paper is a quantitative generalisation of the corollary to Jabin, Otto and Perthame theorem over the class of bounded convex sets.

Theorem 1.1. Let $\epsilon>0$ and $\Omega$ be a convex domain with diameter 2 . Let $u \in$ $W^{2,2}(\Omega)$ be with $u=0$ on $\partial \Omega$ and $\nabla u(x) \cdot \eta_{x}=1$ of $\partial \Omega$ (in the sense of trace) where $\eta_{x}$ is the inward pointing unit normal. Then there exists positive constants $\mathcal{C}>1$ and $\gamma<1$ such that for some $x \in \Omega$,

$$
\left|\Omega \triangle B_{1}(x)\right| \leq \mathcal{C}\left(I_{\epsilon}(u)\right)^{\gamma}
$$

and

$$
\int_{\Omega}\left|\nabla u(z)+\frac{z-x}{|z-x|}\right|^{2} d z \leq \mathcal{C}\left(I_{\epsilon}(u)\right)^{\gamma} .
$$

Corollary 1.2. Let $\epsilon>0$ and $\Omega$ be a convex set of diameter 2 and with $C^{2}$ boundary and curvature bounded above by $\epsilon^{-\frac{1}{2}}$. Let $\Lambda(\Omega):=\left\{u \in W^{2,2}(\Omega): u=0\right.$ on $\partial \Omega$ and $\nabla u(z) \cdot \eta_{z}=1$ for $\left.z \in \partial \Omega\right\}$. There exists positive constants $\mathcal{C}=\mathcal{C}(\Omega)>1$ and $\lambda<1$ such that if $u$ is a minimiser of $I_{\epsilon}$ over $\Lambda(\Omega)$, then

$$
\|u-\zeta\|_{W^{1,2}(\Omega)} \leq \mathcal{C}\left(\epsilon+\inf _{y \in \Omega}\left|\Omega \triangle B_{1}(y)\right|\right)^{\lambda}
$$

where $\zeta(z)=\operatorname{dist}(z, \partial \Omega)$. 
In Theorem 1.1 we take $\gamma=512^{-1}$ and in Corollary $1.2, \lambda=5462^{-1}$. Neither constant is optimal. Corollary 1.2 requires a fair amount of technical work establishing an upper bound for the minimiser of $I_{\epsilon}$ in terms of the 'eccentricity' $\inf _{y \in \Omega, r>0}\left|\Omega \triangle B_{r}(y)\right|$. For the reader primarily interested in the asymptotic behavior of minimisers as $\epsilon \rightarrow 0$ recent powerful results on $\Gamma$-convergence upper bound of $I_{\epsilon}$ (in the case where the function $u$ being approximated satisfies $\left.\nabla u \in B V\left(\Omega: S^{1}\right)\right)$ by Conti and De Lellis [8] and Poliakovsky [23] do much of the work for us and we can give a relatively shorter proof of the following corollary to Theorem 1.1. Note that Corollary 1.3 stated below is a corollary to Corollary 1.2.

Corollary 1.3. Let $\Omega$ be a convex set of diameter 2 with $C^{2}$ boundary. Let $\Lambda(\Omega)$ be as defined in Corollary 1.2. There exists positive constants $\mathcal{C}=\mathcal{C}(\Omega)>1$ and $\lambda<1$ such that if $u^{\epsilon}$ is a minimiser of $I_{\epsilon}$ over $\Lambda(\Omega)$, then

$$
\limsup _{\epsilon \rightarrow 0}\left\|u^{\epsilon}-\zeta\right\|_{W^{1,2}(\Omega)} \leq \mathcal{C}\left(\inf _{y \in \Omega}\left|\Omega \triangle B_{1}(y)\right|\right)^{\lambda}
$$

where $\zeta(z)=\operatorname{dist}(z, \partial \Omega)$

Plan of paper. After the introduction in Section 1 we sketch the proof of the main theorem in Section 2. In Section 3 we prove the main theorem. In Section 4 we establish Corollary 1.3, the additional lemmas needed to establish Corollary 1.2 are given in Section 5.

\subsection{Background}

Given a sequence $\epsilon_{n} \rightarrow 0$ and $u_{n} \in \Lambda(\Omega)$ with limsup $I_{\epsilon_{n}}\left(u_{n}\right)<\infty$, let $u$ be the limit of $u_{n}$. The vector valued measure given by $v_{u}:=\left(\operatorname{div}\left[\Sigma_{1}(\nabla u)\right] \operatorname{div}\left[\Sigma_{2}(\nabla u)\right]\right)$ (where $\Sigma_{1}, \Sigma_{2}$ are the third order polynomial vector fields that satisfy (1.3)) gives us the expression of the limiting energy, i.e. $I(u)=\left\|v_{u}\right\|(\Omega)$. If we consider the 1-dimensional part of the measure

$$
\Gamma:=\left\{x: \limsup _{r \rightarrow 0} \frac{\left\|v_{u}\left(B_{r}(x)\right)\right\|}{r}>0\right\}
$$

it has been shown that $\Gamma$ is 1-rectifiable [9] (see also [10]) and an analogous result has been shown for $M_{\epsilon}$ [6]. It was also shown $\nabla u$ has jump discontinuities across the rectifiable set $\Gamma$ exactly as would be the case if $\nabla u$ was $B V$ and its jump set was given by $\Gamma$. However it is not known (even if $u_{n}$ are the minimisers of $I_{\epsilon_{n}}$ ) if measure $\left\|v_{u}\right\|$ is even singular with respect to Lebesgue measure. Note that for the function $M_{\epsilon}$ the minimiser of the limiting energy is known to be rectifiable [5]. For a sequence with only equibounded energy the measure is not known to be singular.

The original motivation for Theorem 1.1 was to prove a version of it for $\Omega=B_{1}(0)$ without boundary conditions and under the hypotheses $\left.\int_{B_{1}}|1-| \nabla u\right|^{2}|| \nabla^{2} u \mid d z=\beta$, $\left.\int_{B_{1}}|1-| \nabla u\right|^{2} \mid d z \leq \epsilon$ and $\sup \left\{\|u-A\|_{L^{\infty}\left(B_{1}(0)\right)}: A\right.$ is affine with $\left.|\nabla A|=1\right\} \leq$ 
$1000^{-1}$. The conclusion in this case would be that there exists a smooth function $\psi$ with $|\nabla \psi|=1$ everywhere such that $\|\nabla u-\nabla \psi\|_{L^{2}\left(B_{2-1}(0)\right)} \leq c \beta^{\gamma}$ for some $\gamma>0$. This is a kind of quantitative version of the main proposition required to prove compactness in [2], (see Proposition 4.6). The hope is to use such a quantitative result to show $\left\|v_{u}\right\|$ is singular, or at least that $\nabla u$ is continuous at $H^{1}$ a.e. point outside $\Gamma$. We will address these issues in a forthcoming paper [19].

The many strong results about measure $\left\|v_{u}\right\|$ (and the measure that gives the limiting functional for the micromagnetics function) have been achieved by characterising various kinds of blow up of the measure and understanding well the absolute (i.e. non quantitative) situation in the limit $[5,6,9,10,16]$. In some sense there are only two possibilities, either to take a limit and have an absolute situation and to understand the measure from this, or to stop before the limit and have a non-absolute situation and try and understand something about it with a quantitative theorem. Our primary motivation in proving a quantitative version of Jabin-Otto-Perthame Theorem was so as to obtain a result that could be used for the latter approach.

By Poincaré's inequality it is easy to see $\inf _{\Lambda(\Omega)} I_{\epsilon} \geq c \epsilon$ and so Theorem 1.1 follows from the following slightly more general result.

Theorem 1.4. Let $\Omega$ be a convex body centered on 0 with $\operatorname{diam}(\Omega)=2$. Let $\beta>0$, suppose $u: W^{2,2}(\Omega) \rightarrow \mathbb{R}$ is a function satisfying

$$
\left.\int_{\Omega}|1-| \nabla u\right|^{2}|| \nabla^{2} u \mid d z \leq \beta
$$

and

$$
\left.\left.\int_{\Omega}|1-| \nabla u\right|^{2}\right|^{2} d z \leq \beta^{2}
$$

and in addition $u$ satisfies $u=0$ on $\partial \Omega$ and $\nabla u(z) \cdot \eta_{z}=1$ on $\partial \Omega$ in the sense of trace where $\eta_{z}$ is the inward pointing unit normal to $\partial \Omega$ at $z$. and

Then there exists positive constant $\mathcal{C}_{1}>0$ such that $\left|B_{1}(0) \Delta \Omega\right|<\mathcal{C}_{1} \beta^{\frac{1}{512}}$

$$
\int_{\Omega}\left|\nabla u(z)+\frac{z}{|z|}\right|^{2} d z \leq \mathcal{C}_{1} \beta^{\frac{1}{512}}
$$

ACKnowledgements. Part of this paper was written while the author was the Emma e Giovanni Sansone Junior Visitor at Centro di Ricerca Matematica Ennio De Giorgi, Pisa. The hospitality and support of this institute is gratefully acknowledged. I would also like to express my great thanks to the referee for numerous suggestions, simplifications and improvements. The quality of the paper has been substantially increased by the input of the referee. 


\section{Sketch of the proof}

\subsection{Sketch of the proof of Theorem 1.4}

While the proof for convex domains is slightly involved, there are only a couple of ideas that are really central. We will sketch the proof for the case $\Omega=B_{1}(0)$. Ignoring (without comment) many technicalities in order to give an impression of the basic skeleton.

The real engine of the proof is the characterisation in [11] of the set of $\Phi$ such that (1.2) is satisfied. As mentioned in the introduction: as a consequence of the characterisation it was shown there exists a sequence of $\Phi_{n}$ satisfying (1.2) that converge pointwise to the function $\tilde{\Phi}(z)=|z|^{2} e$ for $z \cdot e>0$ and 0 otherwise. Following closely the proof of this, it is possible to extract the existence of functions $\Phi_{\theta}$ and $\Psi_{\theta}$ with $\left\|\nabla \Phi_{\theta}\right\| \leq c \beta^{-\frac{1}{4}},\left\|\Psi_{\theta}\right\| \leq c \beta^{-\frac{1}{4}},\left\|\nabla \Psi_{\theta}\right\| \leq c \beta^{-\frac{1}{2}}$ such that the following two inequalities hold:

Let $\Lambda_{\theta}(z):=\theta$ for $z \cdot \theta>0$ and 0 otherwise,

$$
\left|\Phi_{\theta}(z)-\Lambda_{\theta}(z)\right| \leq c \beta^{\frac{1}{4}} \text { for } z \in N_{\sqrt{\beta}}\left(S^{1}\right) \backslash B_{2 \beta^{\frac{1}{4}}}(\theta)
$$

and (letting $R\left(z_{1}, z_{2}\right)=\left(-z_{2}, z_{1}\right)$ be the anti-clockwise rotation)

$$
\begin{array}{r}
\operatorname{div}\left[\Phi_{\theta}(R(\nabla w))-\Psi_{\theta}(R(\nabla w))\left(1-|R(\nabla w)|^{2}\right)\right] \\
\quad \leq\left. c \beta^{-\frac{1}{2}}|1-| \nabla w\right|^{2}|| \nabla^{2} w \mid \text { for any } w \in W^{2,1} .
\end{array}
$$

Recall for simplicity we have taken $\Omega=B_{1}(0)$, as $\nabla u(z)=-\frac{z}{|z|}$ on $\partial B_{1}(0)$ then we can extend $u$ to a function $\tilde{u}: B_{11 / 10}(0) \rightarrow \mathbb{R}$ such that

$$
\left.\int_{B_{11 / 10}(0)}|1-| \nabla \tilde{u}\right|^{2}|| \nabla^{2} \tilde{u}\left|d z \leq c \beta, \int_{B_{11 / 10}(0)}\right| 1-\left.|\nabla \tilde{u}|^{2}\right|^{2} d z \leq c \beta^{2}
$$

and

$$
\nabla \tilde{u}(z)=-\frac{z}{|z|} \text { for any } z \in B_{11 / 10}(0) .
$$

It is more convenient to work with vector fields that are almost curl free instead of almost divergence free. So notice that (2.1) can be rewritten as

$$
\left|R\left(\Phi_{\theta}(z)\right)-R\left(\Lambda_{\theta}(z)\right)\right| \leq c \beta^{\frac{1}{4}} \text { for } z \in N_{\sqrt{\beta}}\left(S^{1}\right) \backslash B_{2 \beta^{\frac{1}{4}}}(\theta)
$$

and we have $\int_{B_{11 / 10}(0)}\left|\operatorname{curl}\left[R\left(\Phi_{\theta}(R(\nabla \tilde{u}))\right)-R\left(\Psi_{\theta}(R(\nabla \tilde{u}))\right)\left(1-|\nabla \tilde{u}|^{2}\right)\right]\right| \leq$ $c \sqrt{\beta}$. By the quantitative Hodge decomposition type theorem from [2] (Theorem 4.3) we can find a scalar valued function $w_{\theta}$ such that

$$
\begin{aligned}
\int_{B_{11 / 10}(0)} & \left|\nabla w_{\theta}-\left(R\left(\Phi_{\theta}(R(\nabla \tilde{u}))\right)-R\left(\Psi_{\theta}(\nabla R(\nabla \tilde{u}))\right)\left(1-|\nabla \tilde{u}|^{2}\right)\right)\right| d z \\
\leq & c \sqrt{\beta} .
\end{aligned}
$$


The real power of (2.3) is that on the annulus $\mathcal{A}:=B_{11 / 10}(0) \backslash B_{1}(0)$ we know that $\nabla \tilde{u}(z)=-\frac{z}{|z|}$ and hence given inequality (2.2) (and the fact that $|\nabla \tilde{u}|=1$ on $\mathcal{A})$ we have a that $\Phi_{\theta}(R(\nabla \tilde{u}(z))) \in N_{\beta^{\frac{1}{4}}}(\theta)$ for any $z \in \mathcal{A} \cap H(R \theta, 0)$, see Figure 2.1.

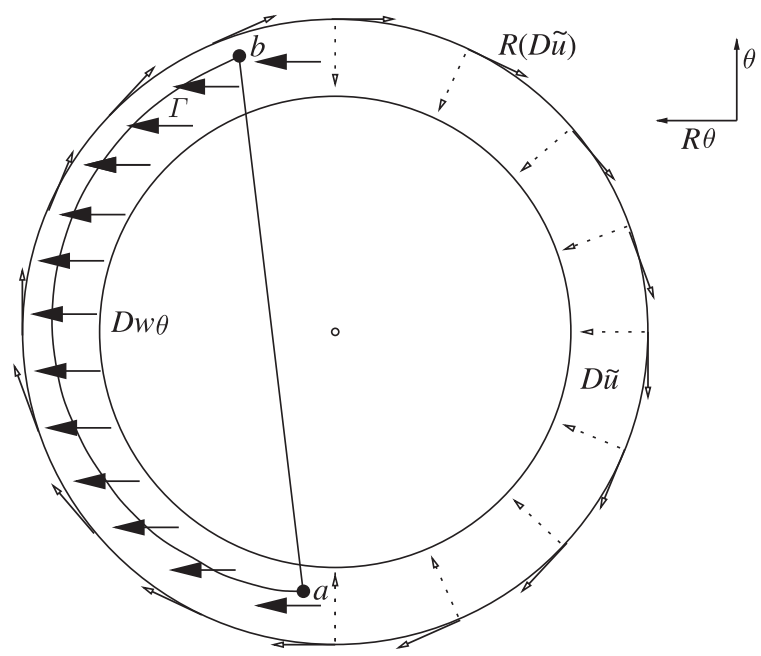

Figure 2.1.

In much the same way in the ball $B_{1}(0)$ by inequalities (2.2), (2.3) and the inequality

$$
\left.\left.\int_{B_{1}(0)}|1-| \nabla \tilde{u}\right|^{2}\right|^{2} \leq \beta^{2}
$$

we have that there exists a large set $\mathcal{G} \subset B_{1}(0) \cap H(0, R \theta)$, with $\left|B_{1}(0) \backslash \mathcal{G}\right| \leq \sqrt{\beta}$ such that if $z \in \mathcal{G}$ then $\nabla w_{\theta}(z) \in B_{\beta^{\frac{1}{4}}}(R \theta)$ or $\nabla w_{\theta}(z) \in B_{\beta_{\frac{1}{4}}}(0)$ depending on whether $R(\nabla u(z)) \cdot \theta>0$ or $R(\nabla u(z)) \cdot \theta \leq 0$.

It is not hard to see we can find points $a, b \in N_{\beta^{\frac{1}{8}}}\left(\langle\theta\rangle \cap \partial B_{1}(0)\right)$ with $|a-b| \sim$ $2, \theta \cdot \frac{b-a}{|b-a|}>0$, the angle between $\frac{b-a}{|b-a|}$ and $\theta$ is at least $\beta^{\frac{1}{8}}$ and $H^{1}([a, b] \backslash \mathcal{G}) \leq$ $\beta^{\frac{1}{4}}$. Let $\mathcal{G}_{1}=\left\{x \in \mathcal{G}: \nabla u(z) \cdot R^{-1}(\theta)>0\right\}$ and $\mathcal{G}_{2}=\mathcal{G} \backslash \mathcal{G}_{1}$. As can be seen from Figure 2.1, we can connect $a$ to $b$ with a path $\Gamma \subset \mathcal{A}$ so

$$
\begin{aligned}
\left|w_{\theta}(b)-w_{\theta}(a)\right| & =\left|\int_{\Gamma} \nabla w_{\theta}(z) t_{z} d H^{1} z\right| \geq\left|R \theta \cdot\left(\int_{\Gamma} t_{z} d H^{1} z\right)\right|-c \beta^{\frac{1}{4}} \\
& =\left|R \theta \cdot \frac{b-a}{|b-a|}\right||b-a|-c \beta^{\frac{1}{4}} .
\end{aligned}
$$


On the other hand

$$
\begin{aligned}
\left|w_{\theta}(b)-w_{\theta}(a)\right| & =\left|\int_{[a, b]} \nabla w_{\theta}(z) \frac{b-a}{|b-a|} d H^{1} z\right| \\
& \leq\left|\int_{[a, b] \cap \mathcal{G}_{1}} \nabla w_{\theta}(z) \frac{b-a}{|b-a|} d H^{1} z\right|+c \beta^{\frac{1}{4}} \\
& \leq\left|\int_{[a, b] \cap \mathcal{G}_{1}} R \theta \cdot \frac{b-a}{|b-a|} d H^{1} z\right|+c \beta^{\frac{1}{4}} \\
& =\left|R \theta \cdot \frac{b-a}{|b-a|}\right| H^{1}\left([a, b] \cap \mathcal{G}_{1}\right)+c \beta^{\frac{1}{4}}
\end{aligned}
$$

and since $\left|R \theta \cdot \frac{b-a}{|b-a|}\right| \geq \beta^{\frac{1}{8}}$ so putting (2.4) and (2.5) together

$$
|a-b| \leq H^{1}\left([a, b] \cap \mathcal{G}_{1}\right)+\frac{c \beta^{\frac{1}{4}}}{\left|R \theta \cdot \frac{b-a}{|b-a|}\right|} \leq H^{1}\left([a, b] \cap \mathcal{G}_{1}\right)+c \beta^{\frac{1}{8}}
$$

So by arguing in the same way for lines parallel to $[a, b]$, by Fubini's theorem we can show $\left|H\left(\frac{a+b}{2}, R\left(\frac{b-a}{|b-a|}\right)\right) \backslash \mathcal{G}_{1}\right| \leq c \beta^{\frac{1}{8}}$. Thus all but $\beta^{\frac{1}{8}}$ points $z \in B_{1}(0) \cap$ $H(0, R(\theta))$ are such that $\nabla u(z) \cdot R^{-1}(\theta)>0$. As $\theta$ is arbitrary we can rephrase this the following way. Given $\phi \in S^{1}$ for all but $\beta^{\frac{1}{8}}$ points $z \in B_{1}(0) \cap H(0, \phi)$ are such that $\nabla u(z) \cdot(-\phi)>0$.

Now take $\psi=\left(\begin{array}{c}\cos \beta^{\frac{1}{16}} \\ \sin \beta^{\frac{1}{16}}\end{array}\right)$. For all but $\beta^{\frac{1}{8}}$ points in $H\left(0, e_{1}\right) \cap H(0,-\psi) \cap$ $H\left(0,-e_{2}\right)$ we have that $\nabla u(z) \cdot\left(-e_{1}\right)>0$ and $\nabla u(z) \cdot \psi>0$, it is not hard to show this implies $\left|\nabla u(z) \cdot e_{1}\right| \leq c \beta^{\frac{1}{16}}$ and since $\nabla u(z) \cdot e_{2}>0$ and $|\nabla u(z)| \sim 1$ we have $\nabla u(z) \in B_{c \beta^{\frac{1}{16}}}\left(e_{2}\right)$ with an exceptional set of measure less than $c \beta^{\frac{1}{8}}$. So integrating a carefully chosen line inside $H\left(0, e_{1}\right) \cap H(0,-\psi) \cap H\left(0,-e_{2}\right)$ and using the fact that $u=0$ on $\partial B_{1}(0)$, we can show $|u(0)-1| \leq c \beta^{\frac{1}{16}}$.

Now, recall $|\nabla u|$ is mostly very close to 1 and we have zero boundary condition. To avoid technicalities let us assume we can apply the coarea formula at 0 so we have

$$
\left.\int_{\theta \in S^{1}} \int_{\mathbb{R}_{+} \theta \cap B_{1}(0)}|| \nabla u(z)\right|^{2}-1 \mid d H^{1} z d H^{1} \theta \leq c \sqrt{\beta} .
$$

Note also that for any $\theta \in S^{1}, u(\theta)=0$ so by the fundamental theorem of Calculus

$$
\begin{aligned}
\left|\int_{\mathbb{R}_{+} \theta \cap B_{1}(0)} \nabla u(z) \cdot(-\theta) d H^{1} z-1\right| & \leq|(u(0)-u(\theta))-1| \\
& \leq c \beta^{\frac{1}{16}}
\end{aligned}
$$


so

$$
\begin{aligned}
\int_{\theta \in S^{1}} \int_{\mathbb{R}_{+} \theta \cap B_{1}(0)}|\nabla u(z)+\theta|^{2} d H^{1} z \theta \\
=\int_{\theta \in S^{1}} \int_{\mathbb{R}_{+} \theta \cap B_{1}(0)}|\nabla u(z)|^{2}+2 \nabla u(z) \cdot \theta+|\theta|^{2} d H^{1} z d H^{1} \theta \\
\leq c \beta^{\frac{1}{16}} .
\end{aligned}
$$

This concludes the sketch of the proof of Theorem 1.4.

\subsection{Sketch of the proof of Corollary 1.2 and Corollary 1.3}

In order to deduce Corollary 1.2 we need to apply Theorem 1.1 to the minimiser of $I_{\epsilon}$ over $\Lambda(\Omega)$. We can only do this if the minimiser has small energy (and from Theorem 1.1 we know it can only have small energy if $\Omega$ is close to a ball). For this reason it is necessary to construct a function in $\Lambda(\Omega)$ with this property. This turns out this is a surprisingly delicate task. It is achieved in Section 4 and Section 5 of the paper.

The obvious way to attempt the construction is to make some adaptation of the function $\zeta(z)=\operatorname{dist}(z, \partial \Omega)$. This function clearly satisfies the correct boundary condition. The first problem is that $\nabla \zeta$ will have its gradient in BV and it is easy to construct examples of convex domains that are close to balls for which the singular part of $\nabla \zeta$ is widely spread over the domain. So it is necessary to convolve $\zeta$. Let $\psi$ denote the convolution of $\zeta$ with a convolution kernel of support size $\sim \epsilon$.

We need to check that the function $\psi$ we obtain by convolving $\zeta$ will have small energy. By recent results of [3] we have that $\nabla \zeta \in S B V\left(\Omega: S^{1}\right)$. So by Poincaré inequality if for most balls the gradient of $\nabla \zeta$ is not too concentrated in balls of sized $\epsilon$ then we would have $\left.\left.\int_{\Omega}|1-| \nabla \psi\right|^{2}\right|^{2} d z$ is small. Now assuming $\Omega$ is close to a ball, then for $x$ not too close to the center of $\Omega$ (which we assume is 0 ) it is not hard to show that $\left|\nabla \zeta(z)+\frac{z}{|z|}\right|$ is small. By convexity of $\Omega$, if $\Phi^{t}$ is a parameterization of $\zeta^{-1}(t)$ then $h \rightarrow \nabla \zeta\left(\Phi^{t}(h)\right)$ will be a monotonic parameterization of $S^{1}$. So the total variation of $\nabla \zeta$ can be explicitly bounded above. The closer $\Omega$ is to a ball the better the estimate on $\left|\nabla \zeta(z)+\frac{z}{|z|}\right|$ holds but near the center it breaks down. To overcome this we do the following. Let $\beta=\left|\Omega \triangle B_{1}(0)\right|$ and let $\eta(z):=1-\beta^{\frac{3}{32}}+|z|$, so $\Pi:=\{z: \eta(z) \leq \zeta(z)\}$ is roughly a ball centered on 0 of radius $\beta^{\frac{3}{32}}$. So defining $w:=\min \{\zeta, \eta\}$ we have $|\nabla w|=1$ a.e. and $\nabla w \in S B V$. Notice that $\int_{J_{\nabla w} \cap \Omega}\left|\nabla w^{+}-\nabla w^{-}\right|^{3} d H^{1} \leq \int_{J_{\nabla \zeta} \backslash \Pi}\left|\nabla \zeta^{+}-\nabla \zeta^{-}\right|^{3} d H^{1}+8 H^{1}(\Gamma)$. Now $\Pi$ is a convex set of diameter approximately $\beta^{\frac{3}{32}}$ so $H^{1}(\Gamma) \sim \beta^{\frac{3}{32}}$. So we have the estimate $\left|\nabla \zeta(z)+\frac{z}{|z|}\right| \leq c \beta^{\frac{3}{32}}$ so $\left|\nabla \zeta^{-}(z)-\nabla \zeta^{+}(z)\right| \leq c \beta^{\frac{3}{32}}$ for any $z \in J_{\nabla \zeta} \backslash \Pi$. Now by convexity of $\Omega$ and hence monotonicity of the gradient along the level set $\zeta^{-1}(t)$ we can prove an explicit upper bound $V(\nabla \zeta, \Omega \backslash \Pi) \leq 8 \pi$. So 
we can estimate

$$
\begin{aligned}
\int_{J_{\nabla \zeta} \backslash \Pi}\left|\nabla \zeta^{+}-\nabla \zeta^{-}\right|^{3} d H^{1} & \leq \sup _{J_{\nabla \zeta} \backslash \Pi}\left|\nabla \zeta^{+}-\nabla \zeta^{-}\right|^{2} \int_{J_{\nabla \zeta} \backslash \Pi}\left|\nabla \zeta^{+}-\nabla \zeta^{-}\right| d H^{1} \\
& \leq \sup _{J_{\nabla \zeta} \backslash \Pi}\left|\nabla \zeta^{+}-\nabla \zeta^{-}\right|^{2} V(\nabla \zeta, \Omega \backslash \Pi) \leq 8 \pi \beta^{\frac{3}{16}}
\end{aligned}
$$

Putting these things together we have $\int_{J_{\nabla w} \cap \Omega}\left|\nabla w^{+}-\nabla w^{-}\right|^{3} d H^{1} \leq c \beta^{\frac{3}{32}}$. This allows us to apply recent results on $\Gamma$-upper bounds of functions whose gradient belongs to $S B V$ by $[8,23]$. These results give the existence of a sequence $u^{\epsilon}$ with the same boundary conditions as $w$ and with the property that $\limsup _{\epsilon \rightarrow 0} I_{\epsilon}\left(u^{\epsilon}\right) \leq$ $c \beta^{\frac{3}{32}}$. This energy bound allows us to apply Theorem 1.1 and hence to establish Corollary 1.3.

To establish Corollary 1.2 requires us to construct a Sobolev function by adapting $w$ with 'our own hands'. Function $\psi$ we obtained by convolving $\zeta$ has a problem in that the convolution will destroy the boundary condition. To circumvent this obstacle, in an $\sqrt{\epsilon}$ neighborhood of the $\partial \Omega$. We convolve the $\zeta$ with a convolution kernel who support decreases in proportion to the distance to the boundary. Let the new function be denoted by $\varphi$. We make the assumption that $\partial \Omega$ is $C^{2}$ with curvature bounded above by $\epsilon^{-\frac{1}{2}}$ and this allows us estimate the various error terms involved in differentiating a function that is convolved with a kernel of varying support. Clearly the goal is to show that $\left.\int_{\Omega} \epsilon^{-1}|1-| \nabla \varphi\right|^{2} \mid d z \leq \beta^{\frac{3}{32}}$ and $\epsilon \int_{\Omega}\left|\nabla^{2} \varphi\right|^{2} d z \leq$ $\beta^{\frac{3}{32}}$. Establishing the upper bounds required in $\Omega \backslash\left(N_{\sqrt{\epsilon}}(\partial \Omega) \cup N_{\epsilon}(\Pi)\right)$ can be achieved by Poincaré inequalities and the estimate $V(\Omega \backslash \Pi, \nabla \zeta) \leq 8 \pi$. Establishing the upper bounds on $N_{\sqrt{\epsilon}}(\partial \Omega)$ can be achieved by very precise estimates on $\nabla \varphi$ and $\nabla^{2} \varphi$ which are made due to the fact that the curvature conditions on $\partial \Omega$ implies $\nabla \zeta$ has no singular points in this neighborhood. The length of $\partial \Pi$ is less than $c \beta \frac{3}{32}$ so as $\|\nabla \varphi\|_{\infty}<c$ we know $\left.\int_{N_{\epsilon}(\partial \Pi)} \epsilon^{-1}|1-| \nabla \varphi\right|^{2} \mid d z \leq c \beta^{\frac{3}{32}}$. Similarly as for $z \in \Omega \backslash N_{\sqrt{\epsilon}}(\partial \Omega),\left\|\nabla^{2} \varphi\right\|_{\infty} \leq c \epsilon^{-1}$ so $\epsilon \int_{N_{\epsilon}(\partial \Pi)}\left|\nabla^{2} \varphi\right|^{2} d z \leq c \beta^{\frac{3}{32}}$. The energy of $\varphi$ in $\Pi \backslash N_{\epsilon}(\partial \Pi)$ can easily be estimated and shown to be negligible so putting these things together gives that $I_{\epsilon}(\varphi) \leq c \beta^{\frac{3}{32}}$. This upper bound allows us to apply Theorem 1.1 and hence to establish Corollary 1.2.

\section{Proof of Theorem}

It should be re-emphasized that the main calculations that make this lemma work (specifically equation (3.7)) are very minor adaptations of the calculations in [11].

Lemma 3.1. Let $\Omega$ be a convex body centered on 0 with $\operatorname{diam}(\Omega) \leq 2$. Suppose $u: W^{2,1}(\Omega) \rightarrow \mathbb{R}$ satisfies (1.4) and (1.5). For each $\theta \in S^{1}$ let $\Lambda_{\theta}: \mathbb{R}^{2} \rightarrow S^{1}$ be 
defined by

$$
\Lambda_{\theta}(z)= \begin{cases}\theta & \text { if } z \cdot \theta>0, \\ 0 & \text { if } z \cdot \theta \leq 0 .\end{cases}
$$

Let $R \in S O(2)$ be the anti-clockwise rotation defined by $R\left(z_{1}, z_{2}\right)=\left(-z_{2}, z_{1}\right)$ and let $m=R(\nabla u)$. We will show there exists a set $\Gamma \subset S^{1}$ with $H^{1}\left(S^{1} \backslash \Gamma\right) \leq 40 \pi \beta^{\frac{1}{8}}$ and $-\Gamma=\Gamma$ such that for any $\theta \in \Gamma$ we can find function $w_{\theta}: \Omega \rightarrow \mathbb{R}$ with the property

$$
\int_{\Omega}\left|\nabla w_{\theta}-R\left(\Lambda_{\theta}(m)\right)\right| \leq c \beta^{\frac{1}{8}}
$$

Proof of Lemma 3.1. Let $M=2\left[\frac{\beta^{-\frac{1}{4}}}{8}\right]$, we divide $S^{1}$ into $M$ disjoint connected subsets of length $\frac{2 \pi}{M}$, denote them $A_{1}, A_{2}, \ldots A_{M}$. We assume they have been ordered sequentially, i.e. $\overline{A_{i}} \cap \overline{A_{i+1}} \neq \emptyset$ for $i=1,2, \ldots M-1$. Also assume they have been ordered so that $-A_{i}=A_{i+\frac{M}{2}}$ for $i=1,2, \ldots \frac{M}{2}$. Let

$$
\mathcal{B}=\left\{k \in\left\{1,2, \ldots \frac{M}{2}\right\}:\left|\left\{x \in \Omega: \frac{\nabla u(x)}{|\nabla u(x)|} \in \overline{A_{k}} \cup \overline{A_{k+\frac{M}{2}}}\right\}\right| \geq \beta^{\frac{1}{8}}\right\} .
$$

Since $\operatorname{Card}(\mathcal{B}) \beta^{\frac{1}{8}} \leq|\Omega| \leq 4 \pi$ we have that $\operatorname{Card}(\mathcal{B}) \leq 4 \pi \beta^{-\frac{1}{8}}$.

Let $\mathcal{D}:=\left\{k \in\left\{2,3, \ldots \frac{M}{2}-1\right\}:\{k-1, k, k+1\} \cap \mathcal{B} \neq \emptyset\right\}$. A simple covering argument shows that $\operatorname{Card}(\mathcal{D}) \leq 20 \pi \beta^{-\frac{1}{8}}$.

Let $\Gamma=\left\{\theta \in S^{1}: \theta \in \bigcup_{k \in\left\{2,3, \ldots \frac{M}{2}-1\right\} \backslash D} \overline{A_{k}} \cup \overline{A_{k+\frac{M}{2}}}\right\}$. Note that for any $\theta \in \Gamma$ we have

$$
\left|\left\{x \in \Omega: \frac{\nabla u(x)}{|\nabla u(x)|} \in B_{2 \beta^{\frac{1}{4}}}(\theta) \cup B_{2 \beta^{\frac{1}{4}}}(-\theta)\right\}\right| \leq 3 \beta^{\frac{1}{8}} .
$$

So pick $\theta \in \Gamma$ without loss of generality we can assume $\theta=e_{1}$. Let $s: \mathbb{R} \rightarrow \mathbb{R}_{+}$ be a smooth monotone function where $s(x)=0$ if $x \leq 0$ and $s(x)=x$ if $x>\beta^{\frac{1}{4}}$ and $\left\|\nabla^{2} s\right\|_{L^{\infty}} \leq \beta^{-\frac{1}{4}}$ and $\left\|\nabla^{3} s\right\|_{L^{\infty}} \leq \beta^{-\frac{1}{2}}$. It is clear such a function exists.

Let $\varphi(z)=s\left(z \cdot e_{1}\right)=s\left(z_{1}\right)$. Define $\Phi: \mathbb{R}^{2} \rightarrow \mathbb{R}^{2}$ by

$$
\begin{aligned}
\Phi(z) & :=\varphi(z)\left(\begin{array}{l}
z_{1} \\
z_{2}
\end{array}\right)+\left(\nabla \varphi(z) \cdot\left(\begin{array}{c}
-z_{2} \\
z_{1}
\end{array}\right)\right)\left(\begin{array}{c}
-z_{2} \\
z_{1}
\end{array}\right) \\
& =\left(\begin{array}{c}
\varphi(z) z_{1}+z_{2}^{2} \varphi_{, 1}(z) \\
\varphi(z) z_{2}-z_{2} z_{1} \varphi_{, 1}(z)
\end{array}\right) .
\end{aligned}
$$

Define

$$
\Psi(z)=\left(\begin{array}{l}
\Psi_{1}(z) \\
\Psi_{2}(z)
\end{array}\right):=\left(\begin{array}{c}
-\varphi, 1(z) \\
\frac{z_{2}}{2} \varphi, 11(z)
\end{array}\right)
$$


Recall $m(z):=R(\nabla u(z))$ so $m$ is divergence free. Note (using the fact $\varphi_{, 2} \equiv 0$ and $\varphi, 12 \equiv 0$ and $\operatorname{div} m \equiv 0$ for the third inequality, and using div $m=0$ for the last inequality)

$$
\begin{aligned}
\operatorname{div}[\Phi(m)]= & \operatorname{div}\left(\begin{array}{c}
\varphi(m) m_{1}+m_{2}^{2} \varphi_{, 1}(m) \\
\varphi(m) m_{2}-m_{2} m_{1} \varphi_{, 1}(m)
\end{array}\right) \\
= & \left(\varphi_{, 1}(m) m_{1,1}+\varphi_{, 2}(m) m_{2,1}\right) m_{1}+\varphi(m) m_{1,1}+2 m_{2} m_{2,1} \varphi_{, 1}(m) \\
& +m_{2}^{2}\left(\varphi, 11(m) m_{1,1}+\varphi_{, 12}(m) m_{2,1}\right) \\
& +\left(\varphi_{, 1}(m) m_{1,2}+\varphi_{, 2}(m) m_{2,2}\right) m_{2} \\
& +\varphi(m) m_{2,2}-\left(\left(m_{1,2} m_{2}+m_{1} m_{2,2}\right) \varphi_{, 1}(m)\right. \\
& \left.+m_{1} m_{2}\left(\varphi, 11(m) m_{1,2}+\varphi, 12(m) m_{2,2}\right)\right) \\
= & m_{1} \varphi_{, 1}(m) m_{1,1}+2 m_{2} m_{2,1} \varphi_{, 1}(m)+m_{2}^{2} m_{1,1} \varphi_{, 11}(m) \\
& +m_{2} m_{1,2} \varphi_{, 1}(m)-\left(\left(m_{1,2} m_{2}+m_{1} m_{2,2}\right) \varphi_{, 1}(m)\right. \\
& +m_{1} m_{2} m_{1,2} \varphi, 11(m) \\
= & 2 \varphi_{, 1}(m)\left(m_{1} m_{1,1}+m_{2} m_{2,1}\right)-\varphi, 11(m) m_{2}\left(m_{1} m_{1,2}+m_{2} m_{2,2}\right) .
\end{aligned}
$$

Note also that

$$
\begin{aligned}
\Psi(m) \cdot \nabla\left(1-|m|^{2}\right)= & -\Psi(m) \cdot\left(\begin{array}{l}
2\left(m_{1} m_{1,1}+m_{2} m_{2,1}\right) \\
2\left(m_{1} m_{1,2}+m_{2} m_{2,2}\right)
\end{array}\right) \\
= & 2 \varphi, 1(m)\left(m_{1} m_{1,1}+m_{2} m_{2,1}\right) \\
& -m_{2} \varphi, 11(m)\left(m_{1} m_{1,2}+m_{2} m_{2,2}\right)
\end{aligned}
$$

so by (3.5) we have

$$
\operatorname{div}[\Phi(m)]=\Psi(m) \cdot \nabla\left(1-|m|^{2}\right) .
$$

Let $\tilde{\Phi}:=R(\Phi)$ and $\tilde{\Psi}:=R(\Psi)$ note $\operatorname{curl}[\tilde{\Phi}(m)] \stackrel{(3.7)}{=} \operatorname{div}[\Phi(m)]=\Psi(m)$. $\nabla\left(1-|m|^{2}\right)$. So

$$
\begin{aligned}
\operatorname{curl}\left[\tilde{\Psi}(m)\left(1-|m|^{2}\right)\right] & =\operatorname{div}[\Psi(m)]\left(1-|m|^{2}\right)+\Psi(m) \cdot \nabla\left(1-|m|^{2}\right) \\
& =\operatorname{div}[\Psi(m)]\left(1-|m|^{2}\right)+\operatorname{curl}[\tilde{\Phi}(m)] .
\end{aligned}
$$

Thus using the fact that $|\nabla \Psi(z)| \leq c|z|\left\|\nabla^{3} \varphi\right\|_{L^{\infty}\left(\mathbb{R}^{2}\right)} \leq c \beta^{-\frac{1}{2}}|z|$ we have

$$
\begin{aligned}
& \operatorname{curl}\left[\tilde{\Phi}(m)-\tilde{\Psi}(m)\left(1-|m|^{2}\right)\right] \\
& \stackrel{(3.8)}{=}-\operatorname{div}[\Psi(m)]\left(1-|m|^{2}\right) \\
& =-\left(\Psi_{1,1}(m) m_{1,1}+\Psi_{1,2}(m) m_{2,1}+\Psi_{2,1}(m) m_{1,2}+\Psi_{2,2}(m) m_{2,2}\right)\left(1-|m|^{2}\right) \\
& \leq\left. c \beta^{-\frac{1}{2}}|m||1-| m\right|^{2}|| \nabla m \mid .
\end{aligned}
$$


Hence

$$
\int_{\Omega}\left|\operatorname{curl}\left[\tilde{\Phi}(m)-\tilde{\Psi}(m)\left(1-|m|^{2}\right)\right]\right| \leq\left. c \beta^{-\frac{1}{2}} \int_{\Omega}|m||1-| m\right|^{2}|| \nabla m \mid
$$

Using (3.9), note that if $x$ is such that $|m(x)| \geq 2$ then for $J(x):=|m(x)|^{3}$ we have $|\nabla J(x)| \leq\left. c|1-| m\right|^{2}|| \nabla m \mid$ and so

$$
\int_{\{x: 2 \leq|m(x)| \leq 4\}}|\nabla J(x)| d x \leq\left. c \int_{\Omega}|1-| m\right|^{2}|| \nabla m \mid \leq c \beta .
$$

Applying the Co-area formula we know $\int_{8}^{64} H^{1}\left(J^{-1}(s)\right) d s \leq c \beta$ and so we must be able to find $t \in[8,64]$ such that $H^{1}\left(J^{-1}(t)\right) \leq c \beta$. Let

$$
\mathcal{G}:=\{x \in \Omega: J(x)<t\}
$$

and define $w: \Omega \rightarrow \mathbb{R}$ by

$$
w(x)= \begin{cases}\tilde{\Phi}(m)-\tilde{\Psi}(m)\left(1-|m|^{2}\right) & \text { for } x \in \mathcal{G} \\ 0 & \text { for } x \in \Omega \backslash \mathcal{G}\end{cases}
$$

So if $x \in \mathcal{G}$,

$$
\begin{gathered}
\operatorname{curl}(w)=\operatorname{curl}\left(\tilde{\Phi}(m)-\tilde{\Psi}(m)\left(1-|m|^{2}\right)\right) \\
\stackrel{(3.9),(3.10)}{\leq} c \beta^{-\frac{1}{2}}|1-| m|||\nabla m| .
\end{gathered}
$$

Thus if $x \in \operatorname{int}(\Omega \backslash \mathcal{G}), \operatorname{curl}\left(\tilde{\Phi}(m)-\tilde{\Psi}(m)\left(1-|m|^{2}\right)\right)=0$.

Since $m \in W^{1,1}(\Omega)$ and $\tilde{\Phi}(x)-\tilde{\Psi}(x)\left(1-|x|^{2}\right)$ is $C^{1}$, the vector field $\tilde{\Phi}(m)-$ $\tilde{\Psi}(m)\left(1-|m|^{2}\right)$ is BV by Theorem 3.94 [4]. So by Theorem 3.83 [4] we have that $w$ is also $\mathrm{BV}$ and the singular part of $\nabla w$, which we denote by $[\nabla w]_{s}$, is supported on $J^{-1}(t) \cap \Omega$. As $|\tilde{\Phi}(m(x))| \leq c|m(x)|^{2}$ and $|\tilde{\Psi}(m(x))| \leq c \beta^{-\frac{1}{4}}|m(x)|$ we have that

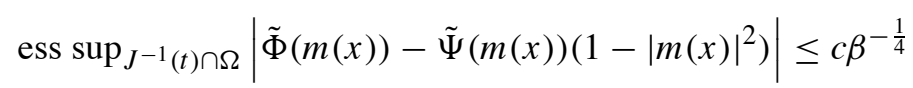

and thus $\left\|[\nabla w]_{S}\right\|(S) \leq c \beta^{-\frac{1}{4}} H^{1}\left(J^{-1}(t) \cap \Omega\right) \leq c \beta^{\frac{3}{4}}$. Now we know that for any set $S \subset \Omega$

$$
\|\operatorname{curl} w\|(S) \leq c\|\nabla w\|(S),
$$

and so in particular

$$
\|\operatorname{curl} w\|\left(J^{-1}(t)\right) \leq c\|\nabla w\|\left(J^{-1}(t)\right) \leq c \beta^{\frac{3}{4}} .
$$


Thus

$$
\begin{aligned}
&\|\operatorname{curl} w\|(\Omega) \leq\|\operatorname{curl} w\|\left(J^{-1}(t)\right)+\|\operatorname{curl} w\|(\mathcal{G})+\|\operatorname{curl} w\|(\operatorname{int}(\Omega \backslash \mathcal{G})) \\
& \stackrel{(3.12),(3.13)}{\leq} c \beta^{\frac{3}{4}}+c \beta^{-\frac{1}{2}} \int_{\mathcal{G}}|1-| m \||\nabla m| \\
& \stackrel{(1.4)}{\leq} c \sqrt{\beta} .
\end{aligned}
$$

Now we try and understand the nature of vector field $\tilde{\Phi}(m(x))-\tilde{\Psi}(m(x))(1-$ $\left.|m(x)|^{2}\right)$. Note that if $z \in N_{\sqrt{\beta}}\left(S^{1}\right) \cap\left\{z_{1}>0\right\} \backslash\left(B_{2 \beta^{\frac{1}{4}}}\left(e_{2}\right) \cup B_{2 \beta^{\frac{1}{4}}}\left(-e_{2}\right)\right)$ then $\varphi(z)=z_{1}, \varphi, 1(z)=1$ and so $\Phi(z) \stackrel{(3.4)}{=}\left(\begin{array}{c}z_{1}^{2}+z_{2}^{2} \\ 0\end{array}\right)$. On the other hand if $z \in$ $N_{\sqrt{\beta}}\left(S^{1}\right) \cap\left\{z_{1} \leq 0\right\} \backslash\left(B_{2 \beta^{\frac{1}{4}}}\left(e_{2}\right) \cup B_{2 \beta^{\frac{1}{4}}}\left(-e_{2}\right)\right)$ then $\varphi(z)=\varphi_{, 1}(z)=0$ and so $\Phi(z)=\left(\begin{array}{l}0 \\ 0\end{array}\right)$.

Now, if $z \in N_{\sqrt{\beta}}\left(S^{1}\right) \cap\left\{z_{1}>0\right\} \backslash\left(B_{2 \beta^{\frac{1}{4}}}\left(e_{2}\right) \cup B_{2 \beta^{\frac{1}{4}}}\left(-e_{2}\right)\right)$ we have

$$
\begin{aligned}
\left|\left(\tilde{\Phi}(z)-\tilde{\Psi}(z)\left(1-|z|^{2}\right)\right)-R\left(\Lambda_{e_{1}}(z)\right)\right| \leq & \left|\tilde{\Phi}(z)-R\left(\Lambda_{e_{1}}(z)\right)\right| \\
& +c \sqrt{\beta} \sup _{z \in N_{\sqrt{\beta}}\left(S^{1}\right)}|\tilde{\Psi}(z)| \\
= & \left|R\left(\begin{array}{c}
z_{1}^{2}+z_{2}^{2} \\
0
\end{array}\right)-R\left(\begin{array}{l}
1 \\
0
\end{array}\right)\right|+c \beta^{\frac{1}{4}} \\
\leq & c \beta^{\frac{1}{4}} .
\end{aligned}
$$

And if we have $z \in N_{\sqrt{\beta}}\left(S^{1}\right) \cap\left\{z_{1} \leq 0\right\} \backslash\left(B_{2 \beta^{\frac{1}{4}}}\left(e_{2}\right) \cup B_{2 \beta^{\frac{1}{4}}}\left(-e_{2}\right)\right)$ arguing in the same way we can conclude

$$
\left|\left(\tilde{\Phi}(z)-\tilde{\Psi}(z)\left(1-|z|^{2}\right)\right)-R\left(\Lambda_{e_{1}}(z)\right)\right| \leq c \beta^{\frac{1}{4}} .
$$

Let $\Pi:=\{z \in \Omega:|m(z)| \in(1-\sqrt{\beta}, 1+\sqrt{\beta})\}$ and let

$$
\mathcal{E}:=\left\{x \in \Omega: \frac{\nabla u(x)}{|\nabla u(x)|} \in B_{2 \beta^{\frac{1}{4}}}\left(e_{1}\right) \cup B_{2 \beta^{\frac{1}{4}}}\left(-e_{1}\right)\right\} .
$$

Note from (3.3) that we know $|\mathcal{E}| \leq 3 \beta^{\frac{1}{8}}$.

Note also $\sqrt{\beta}|\Omega \backslash \Pi| \leq\left. c \int_{\Omega \backslash \Pi}|1-| \nabla u\right|^{2} \mid \stackrel{(1.5)}{\leq} \beta$ thus

$$
|\Omega \backslash \Pi| \leq c \sqrt{\beta}
$$


Now from (3.15) and (3.16)

$$
\left|\int_{\Pi \backslash \mathcal{E}}\left(\tilde{\Phi}(m)-\tilde{\Psi}(m)\left(1-|m|^{2}\right)\right)-R\left(\Lambda_{e_{1}}(m)\right) d z\right| \leq c \beta^{\frac{1}{4}} .
$$

On the other hand recalling the fact that $|\tilde{\Psi}(z)| \leq \beta^{-\frac{1}{4}}|z|,|\tilde{\Phi}(z)| \leq c|z|^{2}$ and using the definition of $\mathcal{G}$ (see (3.10)) we have

$$
\begin{aligned}
\mid \int_{\mathcal{G} \backslash \Pi} & \left(\left(\tilde{\Phi}(m)-\tilde{\Psi}(m)\left(1-|m|^{2}\right)\right)-R\left(\Lambda_{e_{1}}(m)\right)\right) d z \mid \\
& \leq c|\mathcal{G} \backslash \Pi| \\
& \quad \leq c \sqrt{\beta} .
\end{aligned}
$$

Thus applying (3.19) to (3.20) gives

$$
\left|\int_{\mathcal{G} \backslash \mathcal{E}}\left(\left(\tilde{\Phi}(m)-\tilde{\Psi}(m)\left(1-|m|^{2}\right)\right)-R\left(\Lambda_{e_{1}}(m)\right)\right) d z\right| \leq c \beta^{\frac{1}{4}} .
$$

Recall that $|\mathcal{E}| \leq 3 \beta^{\frac{1}{8}}$ so

$$
\begin{aligned}
\left|\int_{\mathcal{E} \cap \mathcal{G}}\left(\left(\tilde{\Phi}(m)-\tilde{\Psi}(m)\left(1-|m|^{2}\right)\right)-R\left(\Lambda_{e_{1}}(m)\right)\right) d z\right| & \leq c|\mathcal{E}| \\
& \leq c \beta^{\frac{1}{8}}
\end{aligned}
$$

Putting this inequality together with (3.21) gives

$$
\left|\int_{\mathcal{G}}\left(\left(\tilde{\Phi}(m)-\tilde{\Psi}(m)\left(1-|m|^{2}\right)\right)-R\left(\Lambda_{e_{1}}(m)\right)\right) d z\right| \leq c \beta^{\frac{1}{8}} .
$$

So by definition of $w$ (see (3.11)) we have that

$$
\begin{gathered}
\left|\int_{\Omega} w-R\left(\Lambda_{e_{1}}(m)\right) d z\right| \stackrel{(3.22)}{\leq} c \beta^{\frac{1}{8}}+\left|\int_{\Omega \backslash \mathcal{G}} R\left(\Lambda_{e_{1}}(m)\right) d z\right| \\
\leq c \beta^{\frac{1}{8}}+|\Omega \backslash \mathcal{G}| \\
\stackrel{(3.18)}{\leq} c \beta^{\frac{1}{8}}
\end{gathered}
$$

Now from (3.14), applying Theorem 4.3 from ( [2]), there exists $w_{e_{1}} \in W^{1,1}(\Omega)$ such that

$$
\int_{\Omega}\left|\nabla w_{e_{1}}-w\right| d z \leq c \beta^{\frac{1}{8}}
$$

thus putting this together with (3.23) and gives (3.2). 
Lemma 3.2. Let $\Omega$ be a convex body centered on 0 and let $u: W^{2,2}(\Omega) \rightarrow \mathbb{R}$ be a function satisfying (1.4) and (1.5) and $u=0$ on $\partial \Omega$ and $\nabla u(z) \cdot \eta_{z}=1$ on $\partial \Omega$ in the sense of trace, where $\eta_{z}$ is the inward pointing unit normal to $\partial \Omega$ at $z$.

For any $r>0$ define $\Omega_{r}:=N_{r}(\Omega)$; we will show we can construct a function $\tilde{u}: W^{2,1}\left(\Omega_{r}\right) \rightarrow \mathbb{R}$ satisfying

$$
\left.\int_{\Omega_{r}}|1-| \nabla \tilde{u}\right|^{2}|| \nabla^{2} \tilde{u}\left|d z \leq \beta, \quad \int_{\Omega_{r}}\right| 1-|\nabla \tilde{u}|^{2} \mid d z \leq \beta,
$$

and

$$
\tilde{u}(z)= \begin{cases}u(z)+r & \text { for } z \in \bar{\Omega} \\ r-d(z, \Omega) & \text { if } z \in \Omega_{r} \backslash \Omega\end{cases}
$$

Proof of Lemma 3.2.

Step 1. We will show $\nabla u(x)=\eta_{x}$ for $H^{1}$ a.e. $x \in \partial \Omega$.

Proof of Step 1. Recall $\nabla u \in W^{1,1}(\Omega)$ and $\nabla u$ is defined on $\partial \Omega$ in the sense of trace, as the trace operator is bounded we know $\int_{\partial \Omega}|\nabla u| d H^{1}<\infty$.

We define

$$
v(z)= \begin{cases}u(z) & \text { for } z \in \bar{\Omega} \\ 0 & \text { if } z \in \Omega_{r} \backslash \Omega .\end{cases}
$$

So note the vector field $\nabla v(z)$ is equal to $\nabla u(z)$ inside $\Omega$ and is zero outside, so by Theorem 3.8 [4] $\nabla v \in B V\left(\Omega_{r}\right)$ and hence by Theorem 3.76 [4] and Theorem 2, Section 5.3 [13] for $H^{1}$ a.e. $x \in \partial \Omega$ the following limits exist

$$
\lim _{\rho \rightarrow 0} f_{B_{\rho}(x) \cap\left\{z:(z-x) \cdot \eta_{x}>0\right\}}|\nabla v(z)-\nabla u(x)| d z=0
$$

and

$$
\lim _{\rho \rightarrow 0} f_{B_{\rho}(x) \cap\left\{z:(z-x) \cdot \eta_{x} \leq 0\right\}}|\nabla v(z)| d z=0 .
$$

Let $w_{x}^{\rho}(z)=\frac{v(x+\rho z)}{\rho}$, by (3.26) and (3.27) for any sequence $\rho_{n} \rightarrow 0$ we have $w_{x}^{\rho_{n}}(z) \stackrel{W^{1,1}}{\rightarrow} w_{x}$ as $n \rightarrow \infty$ where

$$
w_{x}(z)= \begin{cases}\nabla u(x) \cdot z & \text { for } z \in H\left(0, \eta_{x}\right) \\ 0 & \text { for } z \in H\left(0,-\eta_{x}\right)\end{cases}
$$

however $\nabla w_{x}$ would not be curl free unless $\nabla u(x)=\lambda \eta_{x}$ for some $\lambda \in \mathbb{R}$. As we know $\nabla u(x) \cdot \eta_{x}=1$ this implies $\nabla u(x)=\eta_{x}$ for $H^{1}$ a.e. $x \in \partial \Omega$. This completes the proof of Step 1 . 
Step 2. For any $z \in \Omega_{r} \backslash \Omega, \tilde{u}(z)=d\left(z, \partial \Omega_{r}\right)$.

Proof of Step 2. Note that $\|\nabla \tilde{u}\|_{L^{\infty}\left(\Omega_{r} \backslash \Omega\right)} \leq 1$. Let $x \in \partial \Omega_{r}$, let $q(x)$ be the metric projection onto a convex set $\Omega$, i.e. the unique point for which $|x-q(x)|=$ $d(x, \Omega)$. Since $x \in \partial \Omega_{r}=\partial\left(N_{r}(\Omega)\right)=\left\{x \in \Omega^{c}: d(x, \Omega)=r\right\}$ so $|x-q(x)|=r$.

Since $\tilde{u}(x)=0$ and $\tilde{u}(q(x))=r$ and as $\tilde{u}$ is 1-Lipschitz on $\Omega_{r} \backslash \Omega$ this implies $\tilde{u}((1-\alpha) x+\alpha q(x))=\alpha r$ for any $\alpha \in[0,1]$.

Now let $Q(z):=d\left(z, \partial \Omega_{r}\right)$. For every $x \in \partial \Omega_{r}, Q(q(x)) \leq|q(x)-x|=r$. As $\partial \Omega_{r}=\partial\left(N_{r}(\Omega)\right)$ so we know $Q(q(x)) \geq r$ and thus have $Q(q(x))=r$. We also know $Q$ is 1-Lipschitz and $Q(x)=0$, thus in the same way as before $Q((1-\alpha) x+\alpha q(x))=\alpha r$ for any $\alpha \in[0,1]$. Therefor $Q(z)=\tilde{u}(z)$ for any $z \in[x, q(x)], x \in \partial \Omega_{r}$ and this completes the proof of Step 2.

Step 3. We will show that $\tilde{u} \in W^{2,1}\left(\Omega_{r}\right)$ and that $\tilde{u}$ satisfies (3.24).

Proof of Step 3. First we claim that $\tilde{u} \in W^{2,1}\left(\Omega_{r} \backslash \Omega\right)$ and

$$
\int_{\Omega_{r} \backslash \Omega}\left|\nabla^{2} \tilde{u}\right| d z \leq c .
$$

Note that $\tilde{u}(z)=\operatorname{dist}\left(z, \partial \Omega_{r}\right)$ in $\Omega_{r} \backslash \Omega$. By Corollary 1.4 [3] for any compact subset $\Omega^{\prime} \subset \subset \Omega_{r}$ we have $\nabla \tilde{u} \in S B V\left(\Omega^{\prime} \backslash \Omega\right)$. Also as $\tilde{u}(z)=r-\operatorname{dist}(z, \Omega)$ for any $z \in \Omega_{r} \backslash \Omega$ again by Corollary 1.4 [3] for any compact subset $\Omega^{\prime \prime} \subset \subset \mathbb{R}^{2} \backslash \bar{\Omega}$ we have $\nabla \tilde{u} \in S B V\left(\left(\Omega_{r} \backslash \Omega\right) \cap \Omega^{\prime \prime}\right)$. Putting these thing together we have $\nabla \tilde{u} \in$ $S B V\left(\Omega_{r} \backslash \Omega\right)$. Recall $\tilde{u}(x)=r-d(z, \Omega)$ for $z \in \Omega_{r} \backslash \Omega$, so as $\Omega$ is convex for every $z \in \Omega_{r} \backslash \Omega$ there is a unique point $b(z) \in \partial \Omega$ such that $d(z, \Omega)=|b(z)-z|$ and $\nabla \tilde{u}(z)=\frac{b(z)-z}{|b(z)-z|}$. Since $b$ is a continuous function this shows that $\nabla \tilde{u}$ is continuous on $\Omega_{r} \backslash \bar{\Omega}$, hence $S_{\nabla \tilde{u}} \cap \Omega_{r} \backslash \bar{\Omega}=\emptyset$ (recall Definition 3.63 [4]). So by equation (4.2) of Section 4.1 [4] we have that $\nabla \tilde{u} \in W^{1,1}\left(\Omega_{r} \backslash \Omega\right.$ ). Thus in particular (3.28) holds true.

Since $\Omega$ is an extension domain by Theorem 1 , Section 4.4 [13] there exists a function $p: W^{1,2}\left(\mathbb{R}^{2}\right) \rightarrow \mathbb{R}^{2}$ such that $p(z)=\nabla \tilde{u}(z)$ on $\Omega$ and $\operatorname{Spt} p$ is compact. Similarly as $\Omega_{r} \backslash \Omega$ is an extension domain there exists a function $q: W^{1,1}\left(\mathbb{R}^{2}\right) \rightarrow$ $\mathbb{R}^{2}$ such that $q(z)=\nabla \tilde{u}(z)$ on $\Omega_{r} \backslash \Omega$ and Spt $q$ is compact. We define $w: \Omega_{r} \rightarrow \mathbb{R}^{2}$ by $w:=p \mathbb{1}_{\Omega}+q \mathbb{1}_{\Omega_{r} \backslash \Omega}$, by Theorem $3.83[4] w \in B V\left(\Omega_{r}: \mathbb{R}^{2}\right)$ and since $p$ and $q$ agree on $\partial \Omega$ we have that $\nabla w$ as a measure is absolutely continuous with respect to Lebesgue measure (and hence $w \in W^{1,1}\left(\Omega_{r}: \mathbb{R}^{2}\right)$ ) and $\nabla w=\nabla p \mathbb{1}_{\Omega}+\nabla q \mathbb{1}_{\Omega_{r} \backslash \Omega}$. Now as $w=\nabla \tilde{u}$ a.e. in $\Omega_{r}$ we have that $\nabla \tilde{u} \in W^{1,1}\left(\Omega_{r}\right)$.

Since $\nabla^{2} \tilde{u} \in L^{1}$ we know

$$
\begin{aligned}
\left.\int_{\Omega_{r}}|1-| \nabla \tilde{u}\right|^{2}|| \nabla^{2} \tilde{u} \mid d z & =\left.\int_{\Omega}|1-| \nabla \tilde{u}\right|^{2}|| \nabla^{2} \tilde{u}\left|d z+\int_{\Omega_{r} \backslash \Omega}\right| 1-|\nabla \tilde{u}|^{2}|| \nabla^{2} \tilde{u} \mid d z \\
& =\left.\int_{\Omega}|1-| \nabla \tilde{u}\right|^{2}|| \nabla^{2} \tilde{u} \mid d z \\
& \leq \beta .
\end{aligned}
$$

Similarly $\left.\int_{\Omega_{r}}|1-| \nabla \tilde{u}\right|^{2}\left|d z=\int_{\Omega}\right| 1-|\nabla \tilde{u}|^{2} \mid d z \leq \beta$. 
Lemma 3.3. Let $\Omega$ be a convex body with $\operatorname{diam}(\Omega)=2$. Let $u: W^{2,2}(\Omega) \rightarrow \mathbb{R}$ be a function satisfying (1.4) and (1.5) and $u=0$ on $\partial \Omega$ and $\nabla u(z) \cdot \eta_{z}=1$ on $\partial \Omega$ in the sense of trace where $\eta_{z}$ is the inward pointing unit normal to $\partial \Omega$ at $z$. For any $x, v \in \mathbb{R}^{2}$ let $H(x, v):=\left\{z \in \mathbb{R}^{2}:(z-x) \cdot v>0\right\}$.

Let $\Gamma \subset S^{1}$ be the set constructed in Lemma 3.1. Let $\mathcal{U}:=\Omega_{1 / 10}$ be the convex body and $\tilde{u}: W^{2,1}(\mathcal{U}) \rightarrow \mathbb{R}$ be the function constructed in Lemma 3.2. Let $R$ be the anti-clockwise rotation defined by $R\left(z_{1}, z_{2}\right)=\left(-z_{2}, z_{1}\right)$. Let $R_{0} \in\left\{R^{-1}, R\right\}$. There exists a set $\widetilde{\Gamma} \subset \Gamma$ with $H^{1}(\Gamma \backslash \widetilde{\Gamma})=0$ such that for every $\theta \in \widetilde{\Gamma}$ there exists unique points $a_{\theta}, b_{\theta} \in \partial \mathcal{U}$ with $\eta_{a_{\theta}}=\theta$ and $\eta_{b_{\theta}}=-\theta$ with the property that if we define $\mathcal{G}_{\theta}^{R_{0}}:=\left\{z \in \mathcal{U}: \nabla \tilde{u}(z) \cdot R_{0}^{-1} \theta>0\right\}$, then

$$
\left|\mathcal{U} \cap H\left(\frac{a_{\theta}+b_{\theta}}{2}, R_{0}\left(\frac{b_{\theta}-a_{\theta}}{\left|b_{\theta}-a_{\theta}\right|}\right)\right) \backslash \mathcal{G}_{\theta}^{R_{0}}\right| \leq c \beta^{\frac{1}{24}} .
$$

Proof of Lemma 3.3. Without loss of generality assume $\Omega$ is centered on 0, i.e. $\int_{\Omega} z d z=0$. Since $\partial \mathcal{U}$ is smooth and $\mathcal{U}$ is convex there exists a set $\Xi \subset S^{1}$ with $H^{1}\left(S^{1} \backslash \Xi\right)=0$ with the following property:

$\exists$ unique $a_{\varphi} \in \partial \mathcal{U}$ with $\eta_{a_{\varphi}}=\varphi$ and a unique $b_{\varphi} \in \partial \mathcal{U}$ with $\eta_{b_{\varphi}}=-\varphi$ for all $\varphi \in \Xi$.

Now by Lemma 3.2 (3.24) function $\tilde{u}$ satisfies (1.4) and (1.5) so by Lemma 3.1 there exists $\Gamma \subset S^{1}$ with $H^{1}\left(S^{1} \backslash \Gamma\right) \leq 40 \pi \beta^{\frac{1}{8}}$ satisfying (3.2) for every $\theta \in \Gamma$. Define $\widetilde{\Gamma}:=\Gamma \cap \Xi$. Pick $\theta \in \widetilde{\Gamma}$ and let $\varphi:=R R_{0}^{-1} \theta$ so note that $\varphi=\theta$ or $\varphi=-\theta$ depending on whether $R_{0}=R$ or $R_{0}=R^{-1}$.

Note since $\Omega$ is convex $\Omega \subset \overline{H\left(a_{\varphi}, \varphi\right)}$ we also know that $b_{\varphi} \in H\left(a_{\varphi}, \varphi\right)$ (since otherwise given that $\partial \Omega$ is smooth it would not be possible that $\left.\eta_{b_{\varphi}}=-\varphi\right)$, hence defining $\tau_{\varphi}=\frac{b_{\varphi}-a_{\varphi}}{\left|b_{\varphi}-a_{\varphi}\right|}$ we have $\tau_{\varphi} \cdot \varphi>0$.

Let $\tilde{m}=R(\nabla \tilde{u})$, it is easy to see that

$$
\Pi_{\varphi}:=\{z \in \mathcal{U} \backslash \Omega: \tilde{m}(z) \cdot \varphi>0\}=\left\{z \in \mathcal{U} \backslash \Omega: \nabla u(z) \cdot R^{-1} \varphi>0\right\}
$$

forms a connected set whose boundary is contained in $\partial \mathcal{U}$ and $\partial \Omega$ and in two lines parallel to $\varphi$. See Figure 3.1. Note also the endpoints of $\partial \mathcal{U} \cap \overline{\Pi_{\varphi}}$ are given by $a_{\varphi}$ and $b_{\varphi}$.

Since either $\varphi=\theta \in \widetilde{\Gamma}$ or $\varphi=-\theta \in \widetilde{\Gamma}$, we can apply Lemma 3.1. To $\tilde{m}$ and thus there exists function $w_{\varphi}: \mathcal{U} \rightarrow \mathbb{R}$ such that

$$
\int_{\mathcal{U}}\left|\nabla w_{\varphi}-R\left(\Lambda_{\varphi}(\tilde{m})\right)\right| d x \leq c \beta^{\frac{1}{8}}
$$

By the Co-area formula and Chebyshev's inequality there exists a set $H \subset[0,1 / 10]$ such that $H^{1}([0,1 / 10] \backslash H) \leq c \beta^{\frac{1}{24}}$ where

$$
\int_{\tilde{u}^{-1}(t)}\left|\nabla w_{\varphi}-R\left(\Lambda_{\varphi}(\tilde{m})\right)\right| d H^{1} \leq c \beta^{\frac{1}{12}} \text { for all } r \in H .
$$




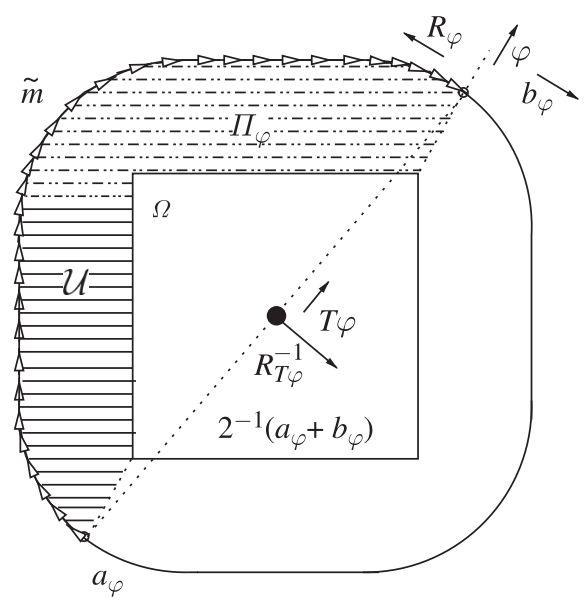

\section{Figure 3.1.}

Pick $s_{0} \in\left[1 / 10-c \beta^{\frac{1}{24}}, 1 / 10\right] \cap H$. Recall $\tau_{\varphi}=\frac{b_{\varphi}-a_{\varphi}}{\left|b_{\varphi}-a_{\varphi}\right|}$ and define

$$
\mathcal{W}_{\varphi}:=\overline{\mathcal{U}} \cap H\left(\frac{a_{\varphi}+b_{\varphi}}{2}, R \tau_{\varphi}\right) \text {. }
$$

We claim that

$$
\partial \mathcal{U} \cap \overline{\Pi_{\varphi}}=\partial \mathcal{U} \cap \overline{\mathcal{W}_{\varphi}} .
$$

Since the endpoints of $\partial \mathcal{U} \cap \overline{\Pi_{\varphi}}$ are the same as the endpoints of $\partial \mathcal{U} \cap \overline{\mathcal{W}_{\varphi}}$ it is sufficient to show $H^{1}\left(\partial \mathcal{U} \cap \overline{\Pi_{\varphi}} \cap \overline{\mathcal{W}_{\varphi}}\right)>0$. Let

$$
\Lambda=\sup \left\{\lambda>0:\left(\frac{a_{\varphi}+b_{\varphi}}{2}+\lambda R \tau_{\varphi}+\left\langle\tau_{\varphi}\right\rangle\right) \cap \partial \mathcal{U} \neq \emptyset\right\}
$$

then let $c_{\varphi}$ be the point given by $\left(\frac{a_{\varphi}+b_{\varphi}}{2}+\Lambda R \tau_{\varphi}+\left\langle\tau_{\varphi}\right\rangle\right) \cap \partial \mathcal{U}$. Since $\partial \mathcal{U}$ is smooth $\eta_{c_{\varphi}}=R^{-1} \tau_{\varphi}$, so $\nabla u\left(c_{\varphi}\right)=R^{-1} \tau_{\varphi}$ and thus $\nabla u\left(c_{\varphi}\right) \cdot R^{-1} \varphi=R^{-1} \tau_{\varphi} \cdot R^{-1} \varphi=\tau_{\varphi}$. $\varphi>0$. As this inequality is strict, in a neighborhood of $c_{\varphi}$ the same inequality will be satisfied. Thus we have $H^{1}\left(\partial \mathcal{U} \cap \overline{\Pi_{\varphi}} \cap \overline{\mathcal{W}_{\varphi}}\right)>0$ and so we have established (3.33).

By the construction of $\Pi_{\varphi}, \mathcal{W}_{\varphi}$ and by (3.33) and the choice of $s_{0} \in\left[\frac{1}{10}-\right.$ $\left.c \beta^{\frac{1}{24}}, \frac{1}{10}\right]$ we have

$$
H^{1}\left(\partial \Omega_{s_{0}} \cap \overline{\Pi_{\varphi}} \Delta \overline{\mathcal{W}_{\varphi}}\right) \leq c \beta^{\frac{1}{24}}
$$

There must exist $\psi \in\left(0,2 \beta^{\frac{1}{24}}\right)$ such that, defining $Q:=\left(\begin{array}{cc}\cos \psi & -\sin \psi \\ \sin \psi & \cos \psi\end{array}\right)$, we have

$$
\left|R \varphi \cdot Q \tau_{\varphi}\right|>\beta^{\frac{1}{24}}
$$


Let $\zeta_{\varphi}:=\frac{a_{\varphi}+b_{\varphi}}{2}+\mathcal{C}_{2} \beta^{\frac{1}{24}} R \tau_{\varphi}$. From the construction it is clear that we can choose constant $\mathcal{C}_{2}$ large enough so that

$$
\operatorname{Card}\left(\partial \Omega_{s_{0}} \cap H\left(\frac{a_{\varphi}+b_{\varphi}}{2}, R \tau_{\varphi}\right) \cap\left\{\zeta_{\varphi}+\left\langle Q \tau_{\varphi}\right\rangle\right\}\right)=2 .
$$

Let

$$
\mathfrak{A}:=\sup \left\{t>0: \partial \Omega_{s_{0}} \cap\left\{\zeta_{\varphi}+t R \tau_{\varphi}+\left\langle Q \tau_{\varphi}\right\rangle\right\} \neq \emptyset\right\} .
$$

For $t \in(0, \mathfrak{A})$ let $\varrho_{t}^{1}, \varrho_{t}^{2}$ be the points defined by $\left\{\varrho_{t}^{1}, \varrho_{t}^{2}\right\}=\partial \Omega_{s_{0}} \cap\left\{\zeta_{\varphi}+t R \tau_{\varphi}+\left\langle Q \tau_{\varphi}\right\rangle\right\}$ and $\varrho_{t}^{2} \cdot Q \tau_{\varphi} \geq \varrho_{t}^{1} \cdot Q \tau_{\varphi}$. By (3.34) we can assume constant $\mathcal{C}_{2}$ was chosen large enough so that $\varrho_{t}^{1}, \varrho_{t}^{2} \in \Pi_{\varphi}$. Let $\Sigma_{t}$ be the connected component of $\partial \Omega_{s_{0}} \backslash\left\{\varrho_{t}^{1}, \varrho_{t}^{2}\right\}$ that lies inside $\Pi_{\varphi}$. Thus

$$
\begin{aligned}
& \left|\left(w_{\varphi}\left(\varrho_{t}^{2}\right)-w_{\varphi}\left(\varrho_{t}^{1}\right)\right)-\left(\varrho_{t}^{2}-\varrho_{t}^{1}\right) \cdot R \varphi\right| \\
& \quad=\left|\int_{\Sigma_{t}} \nabla w_{\varphi}(z) \cdot t_{z} d H^{1} z-\int_{\Sigma_{t}} R \varphi \cdot t_{z} d H^{1} z\right| \\
& \quad=\left|\int_{\Sigma_{t}}\left(\nabla w_{\varphi}(z)-R \varphi\right) \cdot t_{z} d H^{1} z\right| \\
& \stackrel{(3.31)}{\leq} c \beta^{\frac{1}{12}} .
\end{aligned}
$$

Let

$$
e_{t}=\int_{\left[\varrho_{t}^{1}, \varrho_{t}^{2}\right]}\left|\nabla w_{\varphi}-R\left(\Lambda_{\varphi}(\tilde{m})\right)\right| d H^{1} x .
$$

By the fundamental theorem of Calculus

$$
\left|\left(w_{\varphi}\left(\varrho_{t}^{2}\right)-w_{\varphi}\left(\varrho_{t}^{1}\right)\right)-\int_{\left[\varrho_{t}^{1}, \varrho_{t}^{2}\right]} R\left(\Lambda_{\varphi}(\tilde{m})\right) \cdot Q \tau_{\varphi} d H^{1} x\right| \leq e_{t} .
$$

Thus in combination with (3.37) we have

$$
\left|\left(\varrho_{t}^{2}-\varrho_{t}^{1}\right) \cdot R \varphi-\int_{\left[\varrho_{t}^{1}, \varrho_{t}^{2}\right]} R\left(\Lambda_{\varphi}(\tilde{m})\right) \cdot Q \tau_{\varphi} d H^{1} x\right| \leq e_{t}+c \beta^{\frac{1}{12}}
$$

Given the definition of $\Lambda_{\varphi}$ (see (3.1)) and of $\mathcal{G}_{\theta}^{R_{0}}$ (see the statement of Lemma 3.3) so

$R\left(\Lambda_{\varphi}(\tilde{m}(x))\right)=R \varphi \Leftrightarrow \tilde{m}(x) \cdot \varphi>0 \Leftrightarrow \nabla \tilde{u}(x) \cdot R^{-1} \varphi>0 \Leftrightarrow \nabla \tilde{u}(x) \cdot R_{0}^{-1} \theta>0 \Leftrightarrow x \in \mathcal{G}_{\theta}^{R_{0}}$.

In exactly the same way $\Lambda_{\varphi}(\tilde{m}(x))=0 \Leftrightarrow x \notin \mathcal{G}_{\theta}^{R_{0}}$. Hence

$$
\int_{\left[\varrho_{t}^{1}, \varrho_{t}^{2}\right]} \Lambda_{\varphi}(\tilde{m}(x)) d H^{1} x=\varphi H^{1}\left(\left[\varrho_{t}^{1}, \varrho_{t}^{2}\right] \cap \mathcal{G}_{\theta}^{R_{0}}\right)
$$


which from (3.39)

$$
\left|\left(\varrho_{t}^{2}-\varrho_{t}^{1}\right) \cdot R \varphi-Q \tau_{\varphi} \cdot R \varphi H^{1}\left(\left[\varrho_{t}^{1}, \varrho_{t}^{2}\right] \cap \mathcal{G}_{\theta}^{R_{0}}\right)\right| \leq e_{t}+c \beta^{\frac{1}{12}}
$$

since (recall (3.35)) we chose $Q$ so that $\left|R \varphi \cdot Q \tau_{\varphi}\right|>\beta^{\frac{1}{24}}$ and since $\frac{\varrho_{t}^{2}-\varrho_{t}^{1}}{\left|\varrho_{t}^{2}-\varrho_{t}^{1}\right|}=Q \tau_{\varphi}$ So

$$
|| \varrho_{t}^{2}-\varrho_{t}^{1}\left|-H^{1}\left(\left[\varrho_{t}^{1}, \varrho_{t}^{2}\right] \cap \mathcal{G}_{\theta}^{R_{0}}\right)\right| \leq c \beta^{-\frac{1}{24}} e_{t}+c \beta^{\frac{1}{24}}
$$

Thus (recall definition (3.36) of $\mathfrak{A}$ )

$$
H^{1}\left(\left[\varrho_{t}^{1}, \varrho_{t}^{2}\right] \cap \mathcal{G}_{\theta}^{R_{0}}\right) \geq\left|\varrho_{t}^{1}-\varrho_{t}^{2}\right|-c \beta^{-\frac{1}{24}} e_{t}-c \beta^{\frac{1}{24}} \text { for any } t \in[0, \mathfrak{A}] .
$$

So

$$
\begin{aligned}
\left|\Omega_{s_{0}} \cap H\left(\zeta_{\varphi}, R\left(Q \tau_{\varphi}\right)\right) \cap \mathcal{G}_{\theta}^{R_{0}}\right| & =\int_{[0, \mathfrak{A}]} H^{1}\left(\left[\varrho_{t}^{1}, \varrho_{t}^{2}\right] \cap \mathcal{G}_{\theta}^{R_{0}}\right) d t \\
& \stackrel{(3.40)}{\geq} \int_{[0, \mathfrak{A}]}\left|\varrho_{t}^{1}-\varrho_{t}^{2}\right|-c \beta^{-\frac{1}{24}} e_{t}-c \beta^{\frac{1}{24}} d t \\
& \stackrel{(3.38)}{\geq}\left|\Omega_{s_{0}} \cap H\left(\zeta_{\varphi}, R\left(Q \tau_{\varphi}\right)\right)\right|-c \beta^{\frac{1}{24}} \\
& -c \beta^{-\frac{1}{24}} \int_{\mathcal{U}}\left|\nabla w_{\varphi}-R\left(\Lambda_{\varphi}(\tilde{m})\right)\right| d x \\
& \stackrel{(3.30)}{\geq}\left|\Omega_{s_{0}} \cap H\left(\zeta_{\varphi}, R\left(Q \tau_{\varphi}\right)\right)\right|-c \beta^{\frac{1}{24}} .
\end{aligned}
$$

Note $\left|\mathcal{U} \backslash \Omega_{s_{0}}\right| \leq c \beta^{\frac{1}{24}}$ and by definition of $\mathcal{W}_{\varphi}$ (see (3.32)) $\left|\mathcal{W}_{\varphi} \backslash H\left(\zeta_{\varphi}, R\left(Q \tau_{\varphi}\right)\right)\right| \leq$ $c \beta^{\frac{1}{24}}$ this together with (3.41) gives $\left|\mathcal{W}_{\varphi} \backslash \mathcal{G}_{\theta}^{R_{0}}\right| \leq c \beta^{\frac{1}{24}}$. Now if $R_{0}=R$ and so $\varphi=\theta$, it is immediate that $\tau_{\varphi}=\frac{b_{\theta}-a_{\theta}}{\left|b_{\theta}-a_{\theta}\right|}$ and so (again recalling definition (3.32)) (3.29) follows. On the other hand if $R_{0}=R^{-1}$ then $\varphi=-\theta$ and so $a_{\varphi}=b_{\theta}, b_{\varphi}=a_{\theta}$, which implies $\tau_{\varphi}=-\frac{b_{\theta}-a_{\theta}}{\left|b_{\theta}-a_{\theta}\right|}$ so $R \tau_{\varphi}=R\left(-\frac{b_{\theta}-a_{\theta}}{\left|b_{\theta}-a_{\theta}\right|}\right)=$ $R^{-1}\left(\frac{b_{\theta}-a_{\theta}}{\left|b_{\theta}-a_{\theta}\right|}\right)=R_{0}\left(\frac{b_{\theta}-a_{\theta}}{\left|b_{\theta}-a_{\theta}\right|}\right)$ hence (again recalling definition (3.32)),(3.29) also follows in this case.

Lemma 3.4. Let $\Omega$ be a convex body with $\operatorname{diam}(\Omega)=2$. Let $u: W^{2,2}(\Omega) \rightarrow \mathbb{R}$ be a function satisfying (1.4) and (1.5) and in addition $u$ satisfies $u=0$ on $\partial \Omega$ and $\nabla u(z) \cdot \eta_{z}=1$ on $\partial \Omega$ in the sense of trace where $\eta_{z}$ is the inward pointing unit normal to $\partial \Omega$ at $z$. Let $a, b \in \Omega$ be such that $\operatorname{diam}(\Omega)=|a-b|$. We will show there exists constant $\mathcal{C}_{3}>1$ and $r_{0} \in\left(\mathcal{C}_{3}^{-1} \beta^{\frac{1}{512}}, \mathcal{C}_{3} \beta^{\frac{1}{512}}\right)$ such that

$$
u(x) \geq 1-\mathcal{C}_{3} \beta^{\frac{1}{512}} \text { for any } x \in \partial B_{r_{0}}\left(\frac{a+b}{2}\right)
$$


Proof of Lemma 4. Let $\mathcal{U}$ be the convex set and $\tilde{u}$ be the function constructed in Lemma 3.3. To simplify our notation we will without loss of generality assume that $\frac{a+b}{2}=0$. It is easy to see we can chose $\tilde{a}, \tilde{b} \in \mathcal{U}$ such that $\frac{\tilde{a}-\tilde{b}}{|\tilde{a}-\tilde{b}|}=\frac{a-b}{|a-b|}$, $|\tilde{a}-\tilde{b}|=\operatorname{diam}(\mathcal{U})$ and $\frac{\tilde{a}+\tilde{b}}{2}=0$. Without loss of generality also assume $\frac{\tilde{a}-\tilde{b}}{|\tilde{a}-\tilde{b}|}=$ $e_{2}$. For any $z \in \partial \mathcal{U}$ let $\eta_{z}$ denote the inward pointing unit normal to $\partial \mathcal{U}$ at $z$. Note that $\eta_{\tilde{a}}=-e_{2}$ since otherwise $\mathcal{U} \not \subset B_{|\tilde{a}-\tilde{b}|}(\tilde{b})$ and this contradicts the fact that $|\tilde{a}-\tilde{b}|=\operatorname{diam}(\mathcal{U})$. For the same reason $\eta_{\tilde{b}}=e_{2}$.

Step 1. Let $P:\left[0, H^{1}(\partial \mathcal{U})\right) \rightarrow \partial \mathcal{U}$ be a 'clockwise' parameterisation of $\partial \mathcal{U}$ by arclength with $P(0)=\tilde{a}$. For some $\gamma_{1} \in\left(H^{1}(\partial \mathcal{U})-2 \beta^{\frac{1}{512}}, H^{1}(\partial \mathcal{U})-\beta^{\frac{1}{256}}\right)$ and $\gamma_{2} \in\left(\beta^{\frac{1}{256}}, 2 \beta^{\frac{1}{512}}\right)$ we have that for $\sigma_{1}=P\left(\gamma_{1}\right), \sigma_{2}=P\left(\gamma_{2}\right)$, (see Figure 3.2) the points $\sigma_{1}, \sigma_{2}$ satisfy the following properties: firstly

$$
\eta_{\sigma_{i}} \in \widetilde{\Gamma} \text { and } \eta_{\sigma_{i}} \cdot\left(-e_{2}\right) \geq 1-c \beta^{\frac{1}{128}} \text { for } i=1,2
$$

secondly

$$
\left|\sigma_{1}-\sigma_{2}\right| \leq 40 \beta^{\frac{1}{512}}
$$

and thirdly

$$
\sigma_{1} \cdot\left(-e_{1}\right) \geq \frac{\beta^{\frac{1}{256}}}{2} \text { and } \sigma_{2} \cdot e_{1} \geq \frac{\beta^{\frac{1}{256}}}{2}
$$

Proof of Step 1. Recall $\mathcal{U}=\Omega_{\frac{1}{10}}(\Omega)$, so for any $x \in \partial \mathcal{U}$ let $z_{x} \in \partial \Omega$ be such that $d(x, \Omega)=\left|x-z_{x}\right|$, note that we can inscribe a ball $B_{\frac{1}{10}}\left(z_{x}\right) \subset \mathcal{U}$ with $x \in$ $\partial B_{\frac{1}{10}}\left(z_{x}\right) \cap \partial \mathcal{U}$ and $B_{\frac{1}{10}}\left(z_{x}\right) \cap \partial \mathcal{U}=\emptyset$. Thus the curvature of $\partial \mathcal{U}$ is bounded above by 10 and so

$$
\|\ddot{P}\|_{L^{\infty}(\partial \mathcal{U})} \leq 10
$$

Let $\widetilde{\Gamma} \subset S^{1}$ be the set constructed in Lemma 3.3. We will show

$$
\inf \left\{h \in\left[\beta^{\frac{1}{256}}, H^{1}(\partial \mathcal{U})\right]: \eta_{P(h)} \in \widetilde{\Gamma}\right\} \leq 2 \beta^{\frac{1}{512}}
$$

Suppose this is not true: so for every $h \in\left[\beta^{\frac{1}{256}}, 2 \beta^{\frac{1}{512}}\right], \eta_{P(h)} \notin \widetilde{\Gamma}$. Note that since $\partial \mathcal{U}$ is $C^{1},\left\{\eta_{P(h)}: h \in\left[\beta^{\frac{1}{256}}, 2 \beta^{\frac{1}{512}}\right]\right\}$ is connected and since $H^{1}\left(S^{1} \backslash \widetilde{\Gamma}\right) \leq$ $40 \pi \beta^{\frac{1}{8}}$. Thus

$$
H^{1}\left(\left\{\eta_{P(h)}: h \in\left[\beta^{\frac{1}{256}}, 2 \beta^{\frac{1}{512}}\right]\right\}\right) \leq 40 \pi \beta^{\frac{1}{8}}
$$


Note that as $P(0)=e_{2}, \dot{P}(0)=e_{1}$ and as generally for $x \in\left[0, H^{1}(\partial \mathcal{U})\right]$ where we have that $\dot{P}(x)=R\left(\eta_{P(x)}\right)$. So for any $h \in\left[0,2 \beta^{\frac{1}{512}}\right]$

$$
\begin{gathered}
\left|\dot{P}(h)-e_{1}\right| \leq\left|\dot{P}\left(\beta^{\frac{1}{256}}\right)-\dot{P}(0)\right|+\left|\dot{P}(h)-\dot{P}\left(\beta^{\frac{1}{256}}\right)\right| \\
\stackrel{(3.48),(3.46)}{\leq} 20 \beta^{\frac{1}{256}}+40 \pi \beta^{\frac{1}{8}} \leq 40 \pi \beta^{\frac{1}{256}} .
\end{gathered}
$$

Thus by the fundamental theorem of Calculus, $\left|P\left(2 \beta^{\frac{1}{512}}\right)-\left(\tilde{a}+2 \beta^{\frac{1}{512}} e_{1}\right)\right| \leq$ $80 \pi \beta^{\frac{1}{256}} \beta^{\frac{1}{512}}$. Now

$$
\begin{aligned}
\left|\left(\tilde{a}+2 \beta^{\frac{1}{512}} e_{1}\right)-\tilde{b}\right| & =\sqrt{|\tilde{a}-\tilde{b}|^{2}+4 \beta^{\frac{1}{256}}} \\
& \geq|\tilde{a}-\tilde{b}|+\frac{3}{4} \beta^{\frac{1}{256}}
\end{aligned}
$$

Thus $\left|P\left(2 \beta \frac{1}{512}\right)-\tilde{b}\right| \geq|\tilde{a}-\tilde{b}|+\frac{\beta^{\frac{1}{256}}}{2}$ which is a contradiction. Thus we have established (3.47).

Hence (recalling the fact $\left.H^{1}\left(S^{1} \backslash \widetilde{\Gamma}\right) \leq 40 \pi \beta^{\frac{1}{8}}\right)$ we can pick $\gamma_{2} \in\left[\beta^{\frac{1}{256}}, 2 \beta^{\frac{1}{512}}\right] \cap$ $\widetilde{\Gamma}$ such that

$$
\left|\eta_{P\left(\beta^{\left.\frac{1}{256}\right)}\right.}-\eta_{P\left(\gamma_{2}\right)}\right| \leq 50 \pi \beta^{\frac{1}{8}}
$$

and $\eta_{P\left(\gamma_{2}\right)} \in \widetilde{\Gamma}$.

In the same way we can pick $\gamma_{1} \in\left[H^{1}(\partial \mathcal{U})-2 \beta^{\frac{1}{512}}, H^{1}(\partial \mathcal{U})-\beta^{\frac{1}{256}}\right]$ such that $\left|\eta_{P\left(H^{1}(\partial \mathcal{U})-\beta^{\left.\frac{1}{256}\right)}\right.}-\eta_{P\left(\gamma_{1}\right)}\right| \leq 50 \pi \beta^{\frac{1}{8}}$ and $\eta_{P\left(\gamma_{1}\right)} \in \widetilde{\Gamma}$.

Define $\sigma_{2}=P\left(\gamma_{2}\right)$ and $\sigma_{1}=P\left(\gamma_{1}\right)$. Since $\dot{P}(0)=e_{1}$ and recalling again that $\eta_{P(s)}=R^{-1}(\dot{P}(s))$,

$$
\begin{gathered}
\left|\dot{P}(0)-\dot{P}\left(\gamma_{2}\right)\right| \leq\left|\dot{P}(0)-\dot{P}\left(\beta^{\frac{1}{256}}\right)\right|+\left|\dot{P}\left(\beta^{\frac{1}{256}}\right)-\dot{P}\left(\gamma_{2}\right)\right| \\
\underset{(3.46),(3.50)}{\leq} 60 \pi \beta^{\frac{1}{256}} .
\end{gathered}
$$

Arguing in the same way we can establish $\left|\dot{P}(0)-\dot{P}\left(\gamma_{1}\right)\right| \leq 60 \pi \beta^{\frac{1}{256}}$. Thus as $\partial \mathcal{U}$ is convex $\left|\eta_{\sigma_{i}}+e_{2}\right| \leq 60 \pi \beta^{\frac{1}{256}}$ for $i=1,2$ which establishes (3.43). Hence

$$
\sigma_{2} \cdot e_{1}=\left(\sigma_{2}-\tilde{a}\right) \cdot e_{1}=\int_{0}^{\gamma_{2}} \dot{P}(s) \cdot e_{1} d s \stackrel{(3.49)}{\geq}\left(1-40 \pi \beta^{\frac{1}{256}}\right) \gamma_{2} \geq \frac{\beta^{\frac{1}{256}}}{2}
$$


which establishes (3.45) for $\sigma_{2}$. Inequality (3.45) for $\sigma_{1}$ can be established in the same way. Finally note

$$
\left|\sigma_{1}-\sigma_{2}\right|=\left|P\left(\gamma_{2}\right)-P\left(\gamma_{1}\right)\right| \leq \int_{\gamma_{2}}^{H^{1}(\partial \mathcal{U})}|\dot{P}(z)| d z+\int_{0}^{\gamma_{2}}|\dot{P}(z)| d z \leq 40 \beta^{\frac{1}{512}}
$$

which establishes (3.44).

Step 2. For $y \in \mathbb{R}^{2}, \psi \in \mathbb{R}^{2}, \gamma>0$ define $X(y, \psi, \gamma):=\left\{z:\left|\frac{z-y}{|z-y|} \cdot\left(\frac{\psi}{|\psi|}\right)^{\perp}\right| \leq \gamma\right\}$. We will show there exists positive constant $\mathcal{C}_{4}$ and $x_{0} \in N_{\mathcal{C}_{4} \beta \frac{1}{512}}([\tilde{a}, \tilde{b}]) \cap \mathcal{U}$ such that for some $\psi_{0} \in B_{\mathcal{C}_{4} \beta} \frac{1}{256}\left(e_{2}\right)$ the following inequality holds:

$$
\left|X\left(x_{0}, \psi_{0}, \mathcal{C}_{4} \beta^{\frac{1}{256}}\right) \cap \mathcal{U} \backslash\left\{x:\left|\nabla \tilde{u}(x) \cdot e_{1}\right|<\mathcal{C}_{4} \beta^{\frac{1}{256}}\right\}\right| \leq \mathcal{C}_{4} \beta^{\frac{1}{24}}
$$

Proof of Step 2. Recall we know $\sigma_{1}$ and $\sigma_{2}$ are chosen so that $\eta_{\sigma_{1}} \in \widetilde{\Gamma}$ and $\eta_{\sigma_{2}} \in \widetilde{\Gamma}$. We also know $\eta_{\tilde{a}}=-e_{2}$ and $\eta_{\tilde{b}}=e_{2}$. Let $\omega_{1} \in \partial \mathcal{U}$ be the unique point for which $-\eta_{\omega_{1}}=\eta_{\sigma_{1}}$ and let $\omega_{2} \in \partial \mathcal{U}$ be the unique point for which $-\eta_{\omega_{2}}=\eta_{\sigma_{2}}$. See Figure 3.2.

Define

$$
\Pi_{2}:=H\left(\frac{\sigma_{2}+\omega_{2}}{2}, R\left(\frac{\omega_{2}-\sigma_{2}}{\left|\omega_{2}-\sigma_{2}\right|}\right)\right) \cap H\left(\frac{\sigma_{1}+\omega_{1}}{2}, R^{-1}\left(\frac{\omega_{1}-\sigma_{1}}{\left|\omega_{1}-\sigma_{1}\right|}\right)\right)
$$

and

$$
\Pi_{1}:=H\left(\frac{\sigma_{2}+\omega_{2}}{2}, R^{-1}\left(\frac{\omega_{2}-\sigma_{2}}{\left|\omega_{2}-\sigma_{2}\right|}\right)\right) \cap H\left(\frac{\sigma_{1}+\omega_{1}}{2}, R\left(\frac{\omega_{1}-\sigma_{1}}{\left|\omega_{1}-\sigma_{1}\right|}\right)\right)
$$

and let $\Pi=\Pi_{1} \cup \Pi_{2}$ and let $x_{0}:=\overline{\Pi_{1}} \cap \overline{\Pi_{2}}$, see again Figure 3.2.

Let us define $l_{x}^{\theta}:=x+\mathbb{R}_{+} \theta$ for any $x \in \mathbb{R}^{2}, \theta \in S^{1}$. First we will show $\left(x_{0}+\mathbb{R} e_{2}\right) \subset \Pi$ however this inclusion is relatively easy to see because firstly

$$
e_{2} \cdot R\left(\frac{\omega_{1}-\sigma_{1}}{\left|\omega_{1}-\sigma_{1}\right|}\right)=e_{1} \cdot\left(\frac{\omega_{1}-\sigma_{1}}{\left|\omega_{1}-\sigma_{1}\right|}\right) \stackrel{(3.45)}{\geq} \frac{10 \beta^{\frac{1}{256}}}{44}
$$

thus $l_{0}^{e_{2}} \subset H\left(0, R\left(\frac{\omega_{1}-\sigma_{1}}{\left|\omega_{1}-\sigma_{1}\right|}\right)\right)$. And secondly as $x_{0} \in \partial H\left(\frac{\sigma_{1}+\omega_{1}}{2}, R\left(\frac{\omega_{1}-\sigma_{1}}{\left|\omega_{1}-\sigma_{1}\right|}\right)\right)$

$$
l_{x_{0}}^{e_{2}} \subset H\left(x_{0}, R\left(\frac{\omega_{1}-\sigma_{1}}{\left|\omega_{1}-\sigma_{1}\right|}\right)\right)=H\left(\frac{\sigma_{1}+\omega_{1}}{2}, R\left(\frac{\omega_{1}-\sigma_{1}}{\left|\omega_{1}-\sigma_{1}\right|}\right)\right) .
$$

In exactly the same way $l_{x_{0}}^{e_{2}} \subset H\left(\frac{\sigma_{2}+\omega_{2}}{2}, R^{-1}\left(\frac{\omega_{2}-\sigma_{2}}{\left|\omega_{2}-\sigma_{2}\right|}\right)\right)$. Hence $l_{x_{0}}^{e_{2}} \subset \Pi_{1}$. Arguing in the same manner we have $l_{x_{0}}^{-e_{2}} \subset \Pi_{2}$ and thus we have established the claim. 


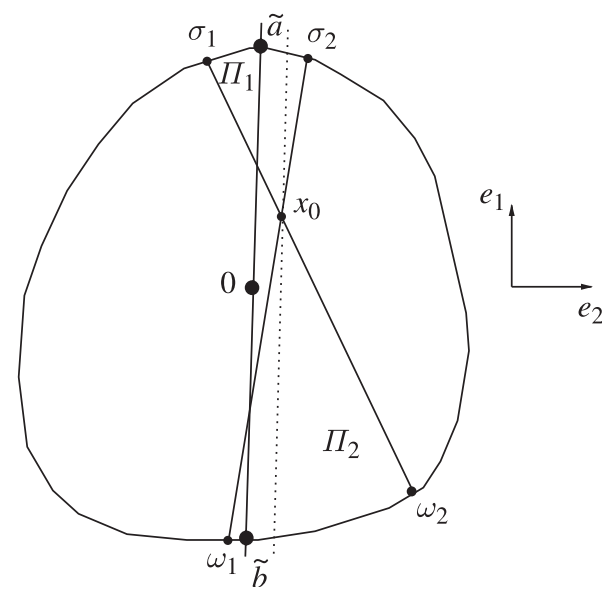

Figure 3.2.

Let $\gamma=l_{x_{0}}^{e_{2}} \cap \partial \mathcal{U}$, by construction we have that $\gamma$ lies in the component of $\partial \mathcal{U}$ between $\sigma_{1}$ and $\sigma_{2}$ and hence by (3.51) we know $d\left(\gamma, l_{0}^{e_{2}}\right) \leq 40 \beta^{\frac{1}{512}}$ and so it follows $x_{0} \in N_{c \beta \frac{1}{512}}([\tilde{a}, \tilde{b}]) \cap \mathcal{U}$.

Since $\eta_{\tilde{a}}=-e_{2}, \eta_{\tilde{b}}=e_{2}$ and $\mathcal{U}$ is convex we know $\omega_{2} \in H\left(0,-e_{1}\right)$ and for the same reasons $\omega_{1} \in H\left(0, e_{1}\right)$ see Figure 3.2. So $\left(\sigma_{2}-\omega_{2}\right) \cdot e_{1} \geq \sigma_{2} \cdot e_{1} \stackrel{\text { (3.45) }}{\geq}$ $c \beta^{\frac{1}{256}}$ and for exactly the same reason $\left(\sigma_{1}-\omega_{1}\right) \cdot\left(-e_{1}\right) \geq \sigma_{1} \cdot\left(-e_{1}\right) \stackrel{(3.45)}{\geq} c \beta^{\frac{1}{256}}$. Thus as $\left|\sigma_{1}-\omega_{1}\right| \leq 2 \operatorname{diam}(\mathcal{U})$ and $\left|\sigma_{2}-\omega_{2}\right| \leq 2 \operatorname{diam}(\mathcal{\mathcal { U }})$ we have $\frac{\sigma_{2}-\omega_{2}}{\left|\sigma_{2}-\omega_{2}\right|} \cdot e_{1} \geq$ $c \beta^{\frac{1}{256}}$ and $\frac{\sigma_{1}-\omega_{1}}{\left|\sigma_{1}-\omega_{1}\right|} \cdot\left(-e_{1}\right) \geq c \beta^{\frac{1}{256}}$. Hence

$$
\begin{aligned}
\left(\frac{\sigma_{1}-\omega_{1}}{\left|\sigma_{1}-\omega_{1}\right|}\right) \cdot\left(\frac{\sigma_{2}-\omega_{2}}{\left|\sigma_{2}-\omega_{2}\right|}\right)= & \left(\frac{\sigma_{1}-\omega_{1}}{\left|\sigma_{1}-\omega_{1}\right|} \cdot e_{1}\right)\left(\frac{\sigma_{2}-\omega_{2}}{\left|\sigma_{2}-\omega_{2}\right|} \cdot e_{1}\right) \\
& +\left(\frac{\sigma_{1}-\omega_{1}}{\left|\sigma_{1}-\omega_{1}\right|} \cdot e_{2}\right)\left(\frac{\sigma_{2}-\omega_{2}}{\left|\sigma_{2}-\omega_{2}\right|} \cdot e_{2}\right) \\
\leq & -c \beta^{\frac{1}{128}}+1 .
\end{aligned}
$$

In other words the angle between $\frac{\sigma_{1}-\omega_{1}}{\left|\sigma_{1}-\omega_{1}\right|}$ and $\frac{\sigma_{2}-\omega_{2}}{\left|\sigma_{2}-\omega_{2}\right|}$ is greater than $\mathcal{C}_{4} \beta^{\frac{1}{256}}$ for some positive constant $\mathcal{C}_{4}$.

Thus there exists $\psi_{0} \in B_{c \beta} \frac{1}{256}\left(e_{2}\right)$ such that $X\left(x_{0}, \psi_{0}, \mathcal{C}_{4} \beta^{\frac{1}{256}}\right) \subset \Pi$. Now since $\eta_{\sigma_{1}}, \eta_{\sigma_{2}} \in \widetilde{\Gamma}$ we can apply Lemma 3.3 so we know that

$$
\left|\mathcal{U} \cap H\left(\frac{\sigma_{2}+\omega_{2}}{2}, R^{-1}\left(\frac{\omega_{2}-\sigma_{2}}{\left|\omega_{2}-\sigma_{2}\right|}\right)\right) \backslash \mathcal{G}_{\eta_{\sigma_{2}}}^{R^{-1}}\right| \leq c \beta^{\frac{1}{24}}
$$


and

$$
\left|\mathcal{U} \cap H\left(\frac{\sigma_{1}+\omega_{1}}{2}, R\left(\frac{\omega_{1}-\sigma_{1}}{\left|\omega_{1}-\sigma_{1}\right|}\right)\right) \backslash \mathcal{G}_{\eta_{\sigma_{1}}}^{R}\right| \leq c \beta^{\frac{1}{24}} .
$$

Thus (recalling the definition of $\Pi_{1},(3.54)$ )

$$
\left|\Pi_{1} \cap \mathcal{U} \backslash \mathcal{G}_{\eta_{\sigma_{2}}}^{R^{-1}} \cap \mathcal{G}_{\eta_{\sigma_{1}}}^{R}\right| \leq c \beta^{\frac{1}{24}} .
$$

In exactly the same way we have (recall (3.53))

$$
\left|\Pi_{2} \cap \mathcal{U} \backslash \mathcal{G}_{\eta_{\sigma_{1}}}^{R^{-1}} \cap \mathcal{G}_{\eta_{\sigma_{2}}}^{R}\right| \leq c \beta^{\frac{1}{24}} .
$$

Now for any $x \in \mathcal{G}_{\eta_{\sigma_{2}}}^{R^{-1}} \cap \mathcal{G}_{\eta_{\sigma_{1}}}^{R}$ we have $\nabla \tilde{u}(x) \cdot R \eta_{\sigma_{2}} \geq 0$ and $\nabla \tilde{u}(x) \cdot R^{-1} \eta_{\sigma_{1}} \geq$ 0 . Since from (3.43) $\eta_{\sigma_{i}} \in X^{+}\left(0,-e_{2}, c \beta^{\frac{1}{256}}\right)$ for $i=1$, 2 we know $R \eta_{\sigma_{2}} \in$ $X^{+}\left(0, e_{1}, c \beta^{\frac{1}{256}}\right)$ and $R^{-1} \eta_{\sigma_{1}} \in X^{+}\left(0,-e_{1}, \beta^{\frac{1}{256}}\right)$, from this it is easy to see (assuming we chose $\mathcal{C}_{4}$ large enough) $\left|\nabla \tilde{u}(x) \cdot e_{1}\right| \leq \mathcal{C}_{4} \beta^{\frac{1}{256}}$. And in the same way for any $x \in \mathcal{G}_{\eta_{\sigma_{1}}}^{R^{-1}} \cap \mathcal{G}_{\eta_{\sigma_{2}}^{R}}^{R}$ we also have $\left|\nabla \tilde{u}(x) \cdot e_{1}\right| \leq \mathcal{C}_{4} \beta^{\frac{1}{256}}$.

$$
\begin{aligned}
& \left|X\left(x_{0}, \psi_{0}, \mathcal{C}_{4} \beta^{\frac{1}{256}}\right) \cap \mathcal{U} \backslash\left\{x:\left|\nabla \tilde{u}(x) \cdot e_{1}\right|<\mathcal{C}_{4} \beta^{\frac{1}{256}}\right\}\right| \\
& \quad \leq c\left|\Pi_{1} \cap \mathcal{U} \backslash \mathcal{G}_{\eta_{\sigma_{1}}}^{R} \cap \mathcal{G}_{\eta_{\sigma_{2}}}^{R^{-1}}\right|+c\left|\Pi_{2} \cap \mathcal{U} \backslash \mathcal{G}_{\eta_{\sigma_{2}}^{R}}^{R} \cap \mathcal{G}_{\eta_{\sigma_{1}}}^{R^{-1}}\right| \\
& \quad \leq \mathcal{C}_{4} \beta^{\frac{1}{24}}
\end{aligned}
$$

which establishes (3.52).

Step 3. There exists positive constant $\mathcal{C}_{5}$ such that for some $v_{1} \in\left\{e_{2},-e_{2}\right\}$ we have

$$
\left|X\left(x_{0}, \psi_{0}, \mathcal{C}_{4} \beta^{\frac{1}{256}}\right) \cap \mathcal{U} \cap H\left(\mathcal{C}_{5} \beta^{\frac{1}{256}} v_{1}, v_{1}\right) \backslash \mathbb{V}_{-v_{1}}\right| \leq \mathcal{C}_{5} \beta^{\frac{1}{24}}
$$

where

$$
\mathbb{V}_{-v_{1}}:=\left\{x \in \mathcal{U}: \nabla \tilde{u}(x) \in N_{\mathcal{C}_{5} \beta \frac{1}{256}}\left(-v_{1}\right)\right\} .
$$

Proof of Step 3. Let $\widetilde{\varpi_{0}}=l_{0}^{-e_{1}} \cap \partial \mathcal{U}$. Note since $\mathcal{U}$ is convex $\eta_{\widetilde{\varpi_{0}}} \cdot e_{1}>0$. We claim

$$
\eta \widetilde{\varpi_{0}} \cdot e_{1}>\frac{1}{10} \text {. }
$$

Suppose this were not the case, then $\eta_{\widetilde{\omega_{0}}} \cdot e_{1} \leq \frac{1}{10}$. Since $\mathcal{U}$ is convex (and recall $\left.\mathcal{U}=\Omega_{\frac{1}{10}}\right)$ and $\operatorname{diam}(\mathcal{U})=\frac{22}{10}$ we know $\mathcal{U} \subset \overline{H\left(\widetilde{\varpi_{0}}, \eta_{\widetilde{\varpi_{0}}}\right)} \subset H\left(-\frac{22}{10} e_{1}, \eta_{\widetilde{\varpi_{0}}}\right)$ 
which implies $\left(\tilde{b}+\frac{22}{10} e_{1}\right) \cdot \eta_{\widetilde{\sigma_{0}}}>0$ and thus

$$
\begin{aligned}
\tilde{b} \cdot e_{2} \sqrt{\frac{99}{100}} & \geq\left(\left(\tilde{b}+\frac{22}{10} e_{1}\right) \cdot e_{2}\right)\left(\eta_{\widetilde{\varpi_{0}}} \cdot e_{2}\right)>-\left(\left(\tilde{b}+\frac{22}{10} e_{1}\right) \cdot e_{1}\right)\left(\eta_{\widetilde{\varpi_{0}}} \cdot e_{1}\right) \\
& =-\frac{22}{10} \eta_{\widetilde{\varpi_{0}}} \cdot e_{1} \geq-\frac{22}{100} .
\end{aligned}
$$

However as $|\tilde{a}-\tilde{b}|=\operatorname{diam}(\mathcal{U})=\frac{22}{10}, \frac{\tilde{a}+\tilde{b}}{2}=0$ and $\frac{\tilde{a}-\tilde{b}}{|\tilde{a}-\tilde{b}|}=e_{2}$ this is a contradiction. Thus (3.57) is established.

Let

$$
\alpha_{0}=\sup \left\{\alpha>0:\left\{\eta_{x}: x \in B_{\alpha}\left(\widetilde{\varpi}_{0}\right) \cap \partial \mathcal{U}\right\} \cap \widetilde{\Gamma}=\emptyset\right\} .
$$

In the case where $\left\{\alpha>0:\left\{\eta_{x}: x \in B_{\alpha}\left(\widetilde{\varpi}_{0}\right) \cap \partial \mathcal{U}\right\} \cap \widetilde{\Gamma}=\emptyset\right\}=\emptyset$ let $\alpha_{0}=0$.

Since $H^{1}\left(S^{1} \backslash \widetilde{\Gamma}\right) \leq 40 \pi \beta^{\frac{1}{8}}$ we know $\partial \mathcal{U} \backslash B_{\alpha_{0}}\left(\widetilde{\varpi_{0}}\right) \neq \emptyset$. Note also

$$
\mathcal{M}_{0}:=\left\{\eta_{x}: x \in B_{\alpha_{0}}\left(\widetilde{\varpi_{0}}\right) \cap \partial \mathcal{U}\right\}
$$

is a connected subset of $S^{1}$. Thus $H^{1}\left(\mathcal{M}_{0}\right) \leq 40 \pi \beta^{\frac{1}{8}}$ and hence for every $z \in$ $B_{\alpha_{0}}\left(\widetilde{\varpi_{0}}\right) \cap \partial \mathcal{U},\left|\eta_{z}-\eta_{\widetilde{\varpi_{0}}}\right| \leq 40 \pi \beta^{\frac{1}{8}}$. So we can pick $\alpha_{1}>\alpha_{0}$ such that some point $\varpi_{0} \in \partial B_{\alpha_{1}}\left(\widetilde{\varpi_{0}}\right) \cap \partial \mathcal{U}$ satisfies $\eta_{\varpi_{0}} \in \widetilde{\Gamma}$ and

$$
\left|\eta_{z}-\eta_{\varpi_{0}}\right| \leq 50 \pi \beta^{\frac{1}{8}} \text { for all } z \in B_{\alpha_{1}}\left(\widetilde{\varpi_{0}}\right) .
$$

Now since $B_{\frac{1}{10}}(0) \subset \mathcal{U}$, we know $\widetilde{\varpi_{0}} \cdot\left(-e_{1}\right) \geq \frac{1}{10}$. Using again the fact that $\eta_{P(s)}=R^{-1}(\dot{P}(s))$ (where $P$ is the parameterisation of $\partial \mathcal{U}$ ) it is easy to see by the fundamental theorem of Calculus that (3.58) implies

$$
\varpi_{0} \cdot\left(-e_{1}\right) \geq \frac{1}{11}
$$

Also from (3.57) and (3.58) we know that

$$
\eta_{\varpi_{0}} \cdot e_{1}>\frac{1}{11}
$$

Let $\varpi_{1} \in \partial \mathcal{U}$ be the unique point for which $\eta_{\varpi_{1}}=-\eta_{\varpi_{0}}$. Note that by (3.60) we know that $\eta_{\varpi_{1}} \cdot\left(-e_{1}\right)>\frac{1}{11}$ and as $\eta_{\tilde{a}}=-e_{2}$ and $\eta_{\tilde{b}}=e_{2}$ by convexity of $\mathcal{U}$ this implies

$$
\varpi_{1} \in \partial \mathcal{U} \cap H\left(0, e_{1}\right) .
$$

Now let $l \in\left(\frac{\varpi_{1}-\varpi_{0}}{\left|\varpi_{1}-\varpi_{0}\right|}\right)^{\perp} \cap S^{1}$ be such that

$$
H^{1}\left([a, b] \cap H\left(\frac{\varpi_{1}+\varpi_{0}}{2}, l\right)\right) \geq \frac{|a-b|}{2} .
$$


Choose $S \in\left\{R^{-1}, R\right\}$ so that $S\left(\frac{\varpi_{1}-\varpi_{0}}{\left|\varpi_{1}-\varpi_{0}\right|}\right)=l$. Since $\eta_{\varpi_{0}} \in \widetilde{\Gamma}$ we can apply Lemma 3.3 and hence we have

$$
\left|\mathcal{U} \cap H\left(\frac{\varpi_{1}+\varpi_{0}}{2}, l\right) \backslash \mathcal{G}_{\eta_{\varpi_{0}}}^{S}\right| \leq c \beta^{\frac{1}{24}} .
$$

From (1.5) and (3.52) we know

$$
\left|X\left(x_{0}, \psi_{0}, \mathcal{C}_{4} \beta^{\frac{1}{256}}\right) \cap \mathcal{U} \backslash\left\{x: \nabla \tilde{u}(x) \in N_{100^{-1}}\left(\left\{e_{2},-e_{2}\right\}\right)\right\}\right| \leq c \beta^{\frac{1}{24}} .
$$

Since so $\left|S^{-1} \eta_{\varpi_{0}} \cdot e_{2}\right| \stackrel{(3.60)}{>} 11^{-1}$ there exists some fixed vector $v_{0} \in\left\{e_{2},-e_{2}\right\}$ such that if $x \in \mathcal{G}_{\eta_{\varpi_{0}}^{S}}^{S} \cap\left\{x: \nabla \tilde{u}(x) \in N_{100^{-1}}\left(\left\{e_{2},-e_{2}\right\}\right)\right\}$ then $\nabla \tilde{u}(x) \in B_{100^{-1}}\left(v_{0}\right)$. So using (3.63) and (3.64)

$$
\begin{aligned}
& \left|X\left(x_{0}, \psi_{0}, \mathcal{C}_{4} \beta^{\frac{1}{256}}\right) \cap \mathcal{U} \cap H\left(\frac{\varpi_{1}+\varpi_{0}}{2}, l\right) \backslash\left\{x: \nabla \tilde{u}(x) \in B_{100^{-1}}\left(v_{0}\right)\right\}\right| \\
& \quad \leq c \beta^{\frac{1}{24}} .
\end{aligned}
$$

Now for any $w \in H\left(0, v_{0}\right)$ we have the elementary inequality $\left|w-v_{0}\right| \leq 4 d\left(w, S^{1}\right)+$ $2\left|w \cdot e_{1}\right|$, so using (1.5), (3.52) and (3.65) we have (assuming constant $\mathcal{C}_{5}$ is large enough, recall definition (3.56))

$$
\left|X\left(x_{0}, \psi_{0}, \mathcal{C}_{4} \beta^{\frac{1}{256}}\right) \cap \mathcal{U} \cap H\left(\frac{\varpi_{1}+\varpi_{0}}{2}, l\right) \backslash \mathbb{V}_{v_{0}}\right| \leq c \beta^{\frac{1}{24}}
$$

By (3.61) $\varpi_{1} \cdot e_{1} \geq 0$ and so $\left|\frac{\varpi_{1}-\varpi_{0}}{\left|\varpi_{1}-\varpi_{0}\right|} \cdot e_{1}\right| \stackrel{(3.59)}{\geq} \frac{1}{44}$ and so $\left|l \cdot e_{2}\right| \geq \frac{1}{44}$. Thus by the fact that $\psi_{0} \in B_{\mathcal{C}_{4} \beta \frac{1}{256}}\left(e_{2}\right)$ and that inequality (3.62) implies $0 \in \overline{H\left(\frac{\varpi_{1}+\varpi_{0}}{2}, l\right)}$ there exists $v_{1} \in\left\{e_{2},-e_{2}\right\}$ such that for some constant $\mathcal{C}_{5}$ we have

$$
X\left(x_{0}, \psi_{0}, \mathcal{C}_{4} \beta^{\frac{1}{256}}\right) \cap H\left(\mathcal{C}_{5} \beta^{\frac{1}{256}} v_{1}, v_{1}\right) \subset H\left(\frac{\varpi_{1}+\varpi_{0}}{2}, l\right) .
$$

Putting (3.67) together with (3.66) gives

$$
\left|X\left(x_{0}, \psi_{0}, \mathcal{C}_{4} \beta^{\frac{1}{256}}\right) \cap \mathcal{U} \cap H\left(\mathcal{C}_{5} \beta^{\frac{1}{256}} v_{1}, v_{1}\right) \backslash \mathbb{V}_{v_{0}}\right| \leq c \beta^{\frac{1}{24}} .
$$

Let $x \in \mathcal{U} \backslash \bar{\Omega} \cap X\left(x_{0}, \psi_{0}, \mathcal{C}_{4} \beta^{\frac{1}{256}}\right) \cap H\left(\mathcal{C}_{5} \beta^{\frac{1}{256}} v_{1}, v_{1}\right)$ so as $\tilde{u}(x)=d(x, \partial \mathcal{U})$ (and since again $\psi_{0} \in B_{\mathcal{C}_{4} \beta \frac{1}{256}}\left(e_{2}\right)$ ) so $\nabla \tilde{u}(x) \in N_{\mathcal{C}_{5} \beta \frac{1}{256}}\left(-v_{1}\right)$ thus we must have $v_{0}=-v_{1}$, this gives $(3.55)$. 
Step 4 . We will show there exists a positive constant $\mathcal{C}_{6}$ such that

$$
\begin{aligned}
& \left(l_{x}^{-\theta} \cup l_{x}^{\theta}\right) \backslash B_{\mathcal{C}_{6} \beta \frac{1}{256}}(x) \subset X\left(x_{0}, \psi_{0}, \mathcal{C}_{4} \beta^{\frac{1}{256}}\right) \\
& \text { for all } x \in B_{\beta \frac{1}{128}}\left(x_{0}\right), \theta \in S^{1} \cap B_{\beta \frac{1}{128}}\left(\psi_{0}\right) .
\end{aligned}
$$

Proof of Step 4. Without loss of generality we assume $x_{0}=0, \psi_{0}=e_{2}$ and $\mathcal{C}_{4}=1$. To begin with to take point $x=\beta^{\frac{1}{128}} e_{1}$, we will show later the general case follows from this. See Figure 3.3.

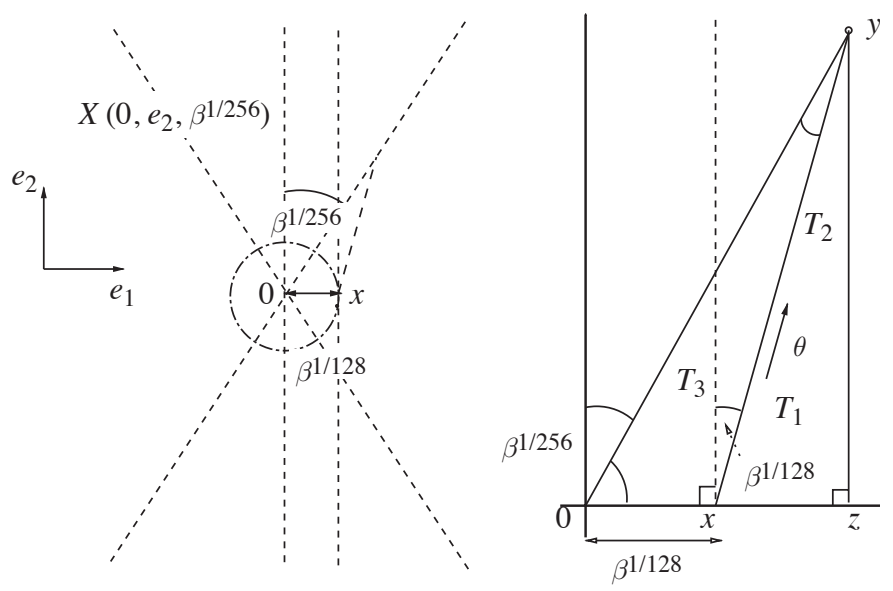

Figure 3.3.

Let $\theta=\left(\begin{array}{c}\sin \beta^{\frac{1}{128}} \\ \cos \beta^{\frac{1}{128}}\end{array}\right)$ and let $y=\partial X\left(0, e_{2}, \beta^{\frac{1}{256}}\right) \cap l_{x}^{\theta}$. We will get an upper bound on $|y|$. Let $z=y \cdot e_{1} e_{1}$. We have two triangles to calculate with: triangle $T_{1}$ with corners on $0, x, y$ which is a subset of triangle $T_{2}$ with corners on $0, z, y$. Note that by applying the law of sins we have $|y|^{-1} \sin \left(\frac{\pi}{2}+\beta^{\frac{1}{128}}\right)=|x-y|^{-1} \sin \left(\frac{\pi}{2}-\beta^{\frac{1}{256}}\right)$. Note that $T_{3}=T_{2} \backslash T_{1}$ is also a right angle triangle and since $|z|=\beta^{\frac{1}{128}}+|x-z|$ we have $|y| \cos \left(\frac{\pi}{2}-\beta^{\frac{1}{256}}\right)=\beta^{\frac{1}{128}}+|y-x| \cos \left(\frac{\pi}{2}-\beta^{\frac{1}{128}}\right)$. Putting this together with the previous equation we have $|y| \sin \beta^{\frac{1}{256}}=\beta^{\frac{1}{128}}+|y| \frac{\cos \beta^{\frac{1}{256}}}{\cos \beta^{\frac{1}{128}}} \sin \beta^{\frac{1}{128}}$ which gives $|y|\left(\sin \beta^{\frac{1}{256}}-\frac{\cos \beta^{\frac{1}{256}}}{\cos \beta^{\frac{1}{128}}} \sin \beta^{\frac{1}{128}}\right)=\beta^{\frac{1}{128}}$. Now by taking the Taylor series approximating sin and cos we have $|y|\left(\beta^{\frac{1}{256}}+O\left(\beta^{\frac{1}{128}}\right)\right)=\beta^{\frac{1}{128}}$. Thus $|y| \sim$ $\beta^{\frac{1}{256}}$ and thus the existence of constant $\mathcal{C}_{6}$ such that (3.68) holds follows instantly for the case $x=\beta^{\frac{1}{128}} e_{1}$.

In the general case where $x \neq \beta^{\frac{1}{128}} e_{1}$ suppose without loss of generality $x$. $e_{1}>0$. Define $\tilde{x}=(x+\langle\theta\rangle) \cap\left\langle e_{1}\right\rangle$, since the angle between $\theta$ and $e_{1}$ is with 
$c \beta^{\frac{1}{256}}$ of $\frac{\pi}{2}$ it is easy to see $\tilde{x} \in B_{2 \beta} \frac{1}{128}(0)$ and of course $l_{\tilde{x}}^{\theta} \cap \partial X\left(0, e_{2}, \beta^{\frac{1}{256}}\right)=$ $l_{x}^{\theta} \cap \partial X\left(0, e_{2}, \beta^{\frac{1}{256}}\right)$ so the argument for the special case $x=\beta^{\frac{1}{128}} e_{1}$ can be applied to show the existence of constant $\mathcal{C}_{6}$ satisfying (3.68).

Step 5. We will establish (3.42).

Proof of Step 5. Let

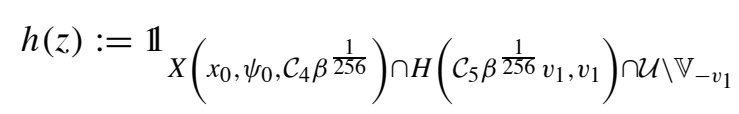

so we know $\int h \stackrel{(3.55)}{\leq} c \beta^{\frac{1}{24}}$. So by Fubini's Theorem,

$$
\begin{aligned}
\int_{\mathcal{U}} \int_{\mathcal{U}}\left(h(z)+\left.\left.\beta^{-1}|1-| \nabla \tilde{u}(z)\right|^{2}\right|^{2}\right)|z-x|^{-1} d z d x \\
\quad \leq \int_{\mathcal{U}}\left(h(z)+\left.\left.\beta^{-1}|1-| \nabla \tilde{u}(z)\right|^{2}\right|^{2}\right)\left(\int_{\mathcal{U}}|z-x|^{-1} d x\right) d z \\
\quad \leq c \int_{\mathcal{U}}\left(h(z)+\left.\left.\beta^{-1}|1-| \nabla \tilde{u}(z)\right|^{2}\right|^{2}\right) d z \\
\quad \stackrel{(1.5)}{\leq} c \beta^{\frac{1}{24}}
\end{aligned}
$$

Let

$$
G:=\left\{x \in B_{\beta^{\frac{1}{128}}}\left(x_{0}\right): \int_{\mathcal{U}}\left(h(z)+\left.\left.\beta^{-1}|1-| \nabla \tilde{u}(z)\right|^{2}\right|^{2}\right)|z-x|^{-1} d z \leq \beta^{\frac{1}{48}}\right\}
$$

so we know $\beta^{\frac{1}{48}}\left|B_{\beta^{\frac{1}{128}}}\left(x_{0}\right) \backslash G\right| \leq c \beta^{\frac{1}{24}}$, thus $\left|B_{\beta^{\frac{1}{128}}}\left(x_{0}\right) \backslash G\right| \leq c \beta^{\frac{1}{48}}$. Assuming $\beta$ is small enough $|G| \geq 2^{-1} \beta^{\frac{1}{64}}$. By Step 4, (3.68) for any $x \in B_{\beta \frac{1}{128}}\left(x_{0}\right), \theta \in$ $B_{\beta^{\frac{1}{128}}}\left(\psi_{0}\right) \cap S^{1}$ we have $\left(l_{x}^{-\theta} \cup l_{x}^{\theta}\right) \backslash B_{\mathcal{C}_{6} \beta^{\frac{1}{256}}}(x) \subset X\left(x_{0}, \psi_{0}, \mathcal{C}_{4} \beta^{\frac{1}{256}}\right)$.

Since $X\left(x_{0}, \psi_{0}, \mathcal{C}_{4} \beta^{\frac{1}{256}}\right)=X\left(x_{0},-\psi_{0}, \mathcal{C}_{4} \beta^{\frac{1}{256}}\right)$ we can assume without loss generality that $\psi_{0} \cdot v_{1}>0$. Pick $x \in G$. By the coarea formula we must be able to find $\theta_{1} \in B_{\beta \frac{1}{128}}\left(\psi_{0}\right) \cap S^{1}$ such that

$$
\int_{\left(l_{x}^{-\theta_{1}} \cup l_{x}^{\theta_{1}}\right) \cap \mathcal{U}} h(z)+\left.\left.\beta^{-1}|1-| \nabla \tilde{u}(z)\right|^{2}\right|^{2} d H^{1} z \leq c \beta^{\frac{1}{48}} / \beta^{\frac{1}{128}} \leq c \beta^{\frac{1}{128}} .
$$

Let $\mathcal{K}:=\left(l_{x}^{-\theta_{1}} \cup l_{x}^{\theta_{1}}\right) \cap \mathcal{U} \cap H\left(\mathcal{C}_{5} \beta^{\frac{1}{256}} v_{1}, v_{1}\right)$. Let $d$, $e$ be the endpoint of $\mathcal{K}$ where we chose $d \in \partial H\left(\mathcal{C}_{5} \beta^{\frac{1}{256}} v_{1}, v_{1}\right)$ and $e \in \partial \mathcal{U}$. As already noted, by Step 
$4 \mathcal{K} \backslash B_{\mathcal{C}_{6} \beta \frac{1}{256}}(x) \stackrel{(3.68)}{\subset} X\left(x_{0}, \psi_{0}, \mathcal{C}_{4} \beta^{\frac{1}{256}}\right) \cap H\left(\mathcal{C}_{5} \beta^{\frac{1}{256}} v_{1}, v_{1}\right) \cap \mathcal{U}$, so for any $z \in$ $\mathcal{K} \backslash B_{\mathcal{C}_{6} \beta \frac{1}{256}}(x)$ with $h(z)=0$ by definition (3.69) we must have $z \in \mathbb{V}_{-v_{1}}$. Thus

$$
H^{1}\left(\mathcal{K} \backslash \mathbb{V}_{-v_{1}}\right) \leq 4 \mathcal{C}_{6} \beta^{\frac{1}{256}}+H^{1}\left(\mathcal{K} \backslash\left(B_{\mathcal{C}_{6} \frac{1}{256}}(x) \cup \mathbb{V}_{-v_{1}}\right)\right) \stackrel{(3.70)}{\leq} c \beta^{\frac{1}{256}}
$$

Note also that if $z \in \mathbb{V}_{-v_{1}}$ so $\nabla \tilde{u}(z) \in B_{\mathcal{C}_{5} \beta \frac{1}{256}}\left(-v_{1}\right)$ and as (recall from Step 2, $\left|\psi_{0} \cdot e_{1}\right|<c \beta^{\frac{1}{256}}$ and we assumed without loss of generality $\psi_{0} \cdot v_{1}>0$ )

$$
\theta_{1} \in B_{\beta^{\frac{1}{128}}}\left(\psi_{0}\right) \subset B_{2 \mathcal{C}_{4} \beta^{\frac{1}{256}}}\left(v_{1}\right)
$$

thus $\nabla \tilde{u}(z) \cdot\left(-\theta_{1}\right) \geq 1+\frac{|\nabla \tilde{u}(z)|^{2}-1}{2}-c \beta^{\frac{1}{128}}$. Now for $z \in \mathcal{K}$ let $t_{z}$ denote the tangent to $\mathcal{K}$. Since $t_{z}=-\theta_{1}$ by the fundamental theorem of Calculus

$$
\begin{aligned}
& \tilde{u}(d)-\tilde{u}(e) \geq \int_{\mathbb{V}_{-v_{1}} \cap \mathcal{K}} \nabla \tilde{u}(z) \cdot\left(-\theta_{1}\right) d H^{1} z-\int_{\mathcal{K} \mid \mathbb{V}_{-v_{1}}}|\nabla \tilde{u}(z)| d H^{1} z \\
& \geq\left(1-c \beta^{\frac{1}{128}}\right) H^{1}\left(\mathbb{V}_{-v_{1}} \cap \mathcal{K}\right)-H^{1}\left(\mathcal{K} \backslash \mathbb{V}_{-v_{1}}\right) \\
&\left.\quad c \int_{\mathcal{K}}|1-| \nabla \tilde{u}\right|^{2} \mid d H^{1} z \\
& \stackrel{\text { (3.70),(3.71) }}{\geq}|d-e|\left(1-c \beta^{\frac{1}{256}}\right) .
\end{aligned}
$$

And as the curvature of $\partial \mathcal{U}$ is bounded above by 10 and by (3.72) it is easy to see either $e$ is very close to $\tilde{a}$ or $\tilde{b}$. We will without loss of generality assume the former, so by (3.72) we have

$$
|e-\tilde{a}| \leq c \beta^{\frac{1}{256}} .
$$

It is also easy to see $[e, \tilde{a}] \subset \mathcal{U} \backslash \Omega$ and $\tilde{u}$ is 1-Lipschitz on $\mathcal{U} \backslash \Omega$ so

$$
|\tilde{u}(e)-\tilde{u}(\tilde{a})| \leq c \beta^{\frac{1}{256}} .
$$

Note also as $d \in \partial H\left(\mathcal{C}_{5} \beta^{\frac{1}{256}} v_{1}, v_{1}\right) \cap\left(l_{x}^{-\theta_{1}} \cup l_{x}^{\theta_{1}}\right)$ by (3.72) and the fact that $x \in$ $B_{\beta \frac{1}{128}}\left(x_{0}\right)$ and from Step 2 we know $x_{0} \in N_{c_{4} \beta \frac{1}{512}}([\tilde{a}, \tilde{b}])$, thus $d \in B_{c \beta \frac{1}{512}}(0)$. So we have

$$
\begin{aligned}
& \tilde{u}(d)=\tilde{u}(d)-\tilde{u}(\tilde{a}) \\
& \stackrel{\text { (3.73),(3.74),(3.75) }}{\geq}|d-\tilde{a}|-c \beta^{\frac{1}{256}} \\
& \geq|\tilde{a}|-c \beta^{\frac{1}{512}}=2^{-1} \operatorname{diam}(\mathcal{U})-c \beta^{\frac{1}{512}} .
\end{aligned}
$$

Pick $r_{0} \in\left[|d|+\beta \frac{1}{512},|d|+2 \beta^{\frac{1}{512}}\right]$ such that $\left.\int_{\partial B_{r_{0}}(0)}|1-| \nabla \tilde{u}(z)\right|^{2} \mid d H^{1} z \leq c \beta^{-\frac{1}{512}} \beta$. Now fix $y \in \partial B_{r_{0}}(0)$. Let $s=\mathcal{K} \cap \partial B_{r_{0}}(0)$ and $\Gamma_{1}$ denote a connected component 
of $\partial B_{r_{0}}(0) \backslash\{s, y\}$. So we know $\int_{\Gamma_{1} \cup[d, s]}|\nabla \tilde{u}(z)| d H^{1} z \leq c H^{1}\left(\Gamma_{1} \cup[d, s]\right) \leq$ $c \beta \frac{1}{512}$. Thus we can apply the fundamental theorem of Calculus we have that $|u(y)-u(d)| \leq c \beta^{\frac{1}{512}}$. Since $y$ was an arbitrary point in $\partial B_{r_{0}}(0)$, by using (3.76) this gives

$$
\inf \left\{\tilde{u}(z): z \in \partial B_{r_{0}}(0)\right\} \geq 2^{-1} \operatorname{diam}(\mathcal{U})-c \beta^{\frac{1}{512}} .
$$

By definition $(\operatorname{see}(3.25)) \tilde{u}(z)=u(z)+10^{-1}$ for any $z \in \partial B_{r_{0}}(0)$. Since $\operatorname{diam}(\mathcal{U})=$ $\frac{22}{10}$ putting this with (3.77) we have (3.42).

Proof of Theorem 1.4. Let $r_{0} \in\left(\mathcal{C}_{3}^{-1} \beta^{\frac{1}{512}}, \mathcal{C}_{3} \beta^{\frac{1}{512}}\right)$ be a number. From Lemma 3.4 we obtain that satisfies (3.42).

By Fubini's Theorem we know $\left.\left.\int_{\Omega} \int_{\Omega}|1-| \nabla u(z)\right|^{2}\right|^{2}|z-y|^{-1} d z d y \leq \mathcal{C}_{7} \beta^{2}$ for some constant $\mathcal{C}_{7}>0$. Let

$$
G_{0}:=\left\{y \in \Omega:\left.\left.\int_{\Omega}|1-| \nabla u(z)\right|^{2}\right|^{2}|z-y|^{-1} d z \leq \beta\right\} .
$$

Note that $\left|\Omega \backslash G_{0}\right| \leq \mathcal{C}_{7} \beta$.

Let $a, b \in \bar{\Omega}$ be such that $|a-b|=\operatorname{diam}(\Omega)$. Let $\vartheta=\frac{a+b}{2}$. Since $r_{0}>$ $\mathcal{C}_{3}^{-1} \beta^{\frac{1}{512}}$ we can pick $x_{0} \in B_{\beta^{\frac{1}{4}}}(\vartheta) \cap G_{0} \subset B_{r_{0}}(\vartheta)$. So by the Co-area formula there exists $\Psi \subset S^{1}$ such that $H^{1}\left(S^{1} \backslash \Psi\right) \leq \sqrt{\beta}$ and

$$
\left.\left.\int_{l_{x_{0}}^{\theta} \cap \Omega}|1-| \nabla u\right|^{2}\right|^{2} d H^{1} z \leq c \sqrt{\beta} \text { for each } \theta \in \Psi .
$$

For any $\theta \in S^{1}$ define $P(\theta):=l_{x_{0}}^{\theta} \cap \partial \Omega$, we will show

$$
\left|P(\theta)-x_{0}\right| \geq 1-c \beta^{\frac{1}{512}} \text { for any } \theta \in \Psi .
$$

To see this we argue as follows

$$
\begin{aligned}
u\left(x_{0}\right) & =u\left(x_{0}\right)-u(P(\theta)) \\
& =\int_{\left[x_{0}, P(\theta)\right]} \nabla u(z) \cdot(-\theta) d H^{1} z \\
& \stackrel{(3.79)}{\leq}\left|x_{0}-P(\theta)\right|+c \beta^{\frac{1}{4}} .
\end{aligned}
$$

Let $y_{\theta}:=\left[x_{0}, P(\theta)\right] \cap \partial B_{r_{0}}(\vartheta)$. In exactly the same way we have

$$
\left|u\left(y_{\theta}\right)-u\left(x_{0}\right)\right| \leq c \beta^{\frac{1}{512}} .
$$

So

$$
u\left(x_{0}\right) \geq u\left(y_{\theta}\right)-\left|u\left(y_{\theta}\right)-u\left(x_{0}\right)\right| \stackrel{(3.82)}{\geq} u\left(y_{\theta}\right)-c \beta^{\frac{1}{512}} \stackrel{(3.42)}{\geq} 1-c \beta^{\frac{1}{512}} .
$$


This together with (3.81) establishes (3.80).

Let $N=\left[2^{-1} \beta^{-\frac{1}{2}}\right]$, we can divide $S^{1}$ into $N$ disjoint pieces of equal length, denote them $I_{1}, I_{2}, \ldots I_{N}$. Formally; $\bigcup_{k=1}^{N} I_{k}=S^{1}$ and $H^{1}\left(I_{k}\right)=\frac{2 \pi}{N}$ for each $k=1,2, \ldots N$. We can pick $\theta_{k} \in I_{k} \cap \Psi$ for each $k=1,2, \ldots N$.

Let

$$
h=\min \left\{\left|P\left(\theta_{k}\right)-x_{0}\right|: k \in\{1,2, \ldots N\}\right\} .
$$

We define $\Pi$ to be the convex hull of the points $x_{0}+h \theta_{1}, x_{0}+h \theta_{2}, \ldots x_{0}+h \theta_{N}$. Now by the construction of $\Pi$, for any $y \in \partial \Pi$ we can find $k \in\{1,2, \ldots N\}$ such that $\left|y-\left(x_{0}+h \theta_{k}\right)\right| \leq c \sqrt{\beta}$ and thus $\left|y-x_{0}\right| \geq h-c \sqrt{\beta}$ and so

$$
B_{h-c \sqrt{\beta}}\left(x_{0}\right) \subset \Pi \text {. }
$$

Note that by using (3.80) we know $h>1-c \beta^{\frac{1}{512}}$ and since $\left|x_{0}-\vartheta\right| \leq \beta^{\frac{1}{4}}$ (recalling also that $\Omega$ is convex and so $\Pi \subset \Omega$ ) there exists positive constant $\mathcal{C}_{8}$ such that

$$
B_{1-\mathcal{C}_{8} \beta \frac{1}{512}}(\vartheta) \subset \Omega .
$$

We claim

$$
\Omega \subset B_{1+2 \mathcal{C}_{8} \beta \frac{1}{512}}(\vartheta)
$$

Suppose not, so there exists $y \in \partial \Omega$ such that $|y-\vartheta| \geq 1+2 \mathcal{C}_{8} \beta^{\frac{1}{512}}$. By inequality (3.85) we know $-\frac{y-\vartheta}{|y-\vartheta|}\left(1-\mathcal{C}_{8} \beta \frac{1}{512}\right)+\vartheta \subset \Omega$ and as by convexity of $\Omega$ we know

$$
\begin{aligned}
& {\left[y, \vartheta-\frac{y-\vartheta}{|y-\vartheta|}\left(1-\mathcal{C}_{8} \beta^{\frac{1}{512}}\right)\right] \subset \Omega, \text { thus }} \\
& H^{1}\left(\left[y, \vartheta-\frac{y-\vartheta}{|y-\vartheta|}\left(1-\mathcal{C}_{8} \beta^{\frac{1}{512}}\right)\right]\right) \geq 2+\mathcal{C}_{8} \beta^{\frac{1}{512}}
\end{aligned}
$$

which contradicts the fact $\operatorname{diam}(\Omega)=2$. Hence (3.86) is established. Since the center of mass of $\Omega$ is 0 , i.e. $\int_{\Omega} x d x=0$, by (3.85), (3.86) we have that $|\vartheta| \leq$ $c \beta^{\frac{1}{512}}$. Recall $x_{0} \in B_{\beta^{\frac{1}{4}}}(\vartheta)$ so $\left|x_{0}-P(\theta)\right| \leq|P(\theta)|+\left|x_{0}\right| \stackrel{(3.86)}{\leq} 1+c \beta^{\frac{1}{512}}$ so putting this together with (3.83) we have

$$
u\left(x_{0}\right)-u(P(\theta))=u\left(x_{0}\right) \geq\left|x_{0}-P(\theta)\right|-c \beta^{\frac{1}{512}} .
$$

Thus

$$
\begin{aligned}
\int_{\left[x_{0}, P(\theta)\right]}|\nabla u(z)+\theta|^{2} d H^{1} z & =\int_{\left[x_{0}, P(\theta)\right]}\left(|\nabla u(z)|^{2}+2 \nabla u(z) \cdot \theta+1\right) d H^{1} z \\
& \stackrel{(3.79)}{\leq} 2\left(1+c \beta^{\frac{1}{4}}\right)\left|x_{0}-P(\theta)\right|+2\left(u(P(\theta))-u\left(x_{0}\right)\right) \\
& \stackrel{(3.87)}{\leq} c \beta^{\frac{1}{512}} \text { for any } \theta \in \Psi
\end{aligned}
$$


Now using the elementary fact that $\left|\nabla u(z)+\frac{z-x_{0}}{\left|z-x_{0}\right|}\right|^{2} \leq\left.|| \nabla u(z)\right|^{2}-\left.1\right|^{2}+4$, since $x_{0} \in G_{0}$ we have

$$
\begin{aligned}
\int_{\theta \in S^{1} \backslash \Psi} \int_{l_{x_{0}}^{\theta}} \mid \nabla & u(z)+\left.\frac{z-x_{0}}{\left|z-x_{0}\right|}\right|^{2} d H^{1} z d H^{1} \theta \\
& \leq 4 H^{1}\left(S^{1} \backslash \Psi\right)+\left.\int_{\theta \in S^{1}} \int_{l_{x_{0}}^{\theta}}|| \nabla u(z)\right|^{2}-\left.1\right|^{2} d H^{1} z d H^{1} \theta \\
& \stackrel{(3.78)}{\leq} 5 \sqrt{\beta} .
\end{aligned}
$$

And thus

$$
\begin{aligned}
& \int_{\Omega}\left|\nabla u(z)+\frac{z-x_{0}}{\left|z-x_{0}\right|}\right|^{2} d z \leq c \int_{\Omega}\left|\nabla u(z)+\frac{z-x_{0}}{\left|z-x_{0}\right|}\right|^{2}\left|z-x_{0}\right|^{-1} d z \\
& \leq c \int_{\theta \in S^{1}} \int_{l_{x_{0}}^{\theta}}\left|\nabla u(z)+\frac{z-x_{0}}{\left|z-x_{0}\right|}\right|^{2} d H^{1} z d H^{1} \theta \\
& \stackrel{(3.89),(3.88)}{\leq} c \beta^{\frac{1}{112}} .
\end{aligned}
$$

By Hölder's inequality this gives

$$
\left(\int_{\Omega}\left|\nabla u(z)+\frac{z-x_{0}}{\left|z-x_{0}\right|}\right|^{2} d z\right)^{\frac{1}{2}} \leq c \beta^{\frac{1}{1024}} .
$$

Note that as $x_{0} \in B_{\beta^{\frac{1}{4}}}(\vartheta)$ and (3.84), (3.85) we established that $|\vartheta| \leq c \beta^{\frac{1}{512}}$ so $\left|x_{0}\right| \leq c \beta^{\frac{1}{512}}$. Now for any $z \in \Omega \backslash B_{\beta \frac{1}{1024}}(0)$

$$
\begin{aligned}
\left|\frac{z}{|z|}-\frac{z-x_{0}}{\left|z-x_{0}\right|}\right| & =\left|\frac{z\left|z-x_{0}\right|-\left(z-x_{0}\right)|z|}{|z|\left|z-x_{0}\right|}\right| \\
& =\left|\frac{z\left(\left|z-x_{0}\right|-|z|\right)+x_{0}|z|}{|z|\left|z-x_{0}\right|}\right| \\
& \leq\left|\frac{\left|z-x_{0}\right|-|z|}{\left|z-x_{0}\right|}\right|+\frac{\left|x_{0}\right|}{\left|z-x_{0}\right|} \\
& \leq c \beta \frac{1}{1024} .
\end{aligned}
$$

So

$$
\begin{aligned}
\left(\int_{\Omega}\left|\frac{z}{|z|}-\frac{z-x_{0}}{\left|z-x_{0}\right|}\right|^{2} d z\right)^{\frac{1}{2}} & \leq c \beta^{\frac{1}{512}}+\left(\int_{\Omega \backslash B}\left|\frac{1}{\beta 1024}\right| \frac{z}{|z|}-\left.\frac{z-x_{0}}{\left|z-x_{0}\right|}\right|^{2} d z\right)^{\frac{1}{2}} \\
& \stackrel{(3.91)}{\leq} c \beta^{\frac{1}{1024}} .
\end{aligned}
$$

Putting this together with (3.90) we have (1.6). 


\section{Proof of Corollary 1.3}

We begin by establishing the following proposition.

Proposition 4.1. Let $\Omega$ be a bounded convex domain with $C^{2}$ boundary and $\left|\Omega \triangle B_{1}(0)\right| \leq \beta$. There exists a sequence $u^{\epsilon} \in C^{\infty}(\bar{\Omega})$ such that $u^{\epsilon}(z)=0$, $\nabla u^{\epsilon}(z) \cdot \eta_{z}=1$ for $z \in \partial \Omega$ (where $\eta_{z}$ is the inward pointing unit normal to $\partial \Omega$ at z) and for which

$$
\left.\left.\limsup _{\epsilon \rightarrow 0} \int_{\Omega} \epsilon^{-1}|1-| \nabla u^{\epsilon}\right|^{2}\right|^{2}+\epsilon\left|\nabla^{2} u^{\epsilon}\right|^{2} d z \leq c \beta^{\frac{3}{32}} .
$$

\subsection{Proof of Proposition 4.1}

Lemma 4.2. Suppose $\Omega$ is a convex and $\left|\Omega \triangle B_{1}(0)\right|=\beta$. For $a_{\theta}=\partial \Omega \cap l_{0}^{\theta}$ the following inequality holds true

$$
|| a_{\theta}|-1| \leq c \sqrt{\beta} \text { for any } \theta \in S^{1} \text { and so } \partial \Omega \subset N_{c \sqrt{\beta}}\left(\partial B_{1}(0)\right) .
$$

In addition there exists constant $c$ such that

$$
\left|\eta_{a_{\theta}}+\theta\right| \leq c \beta^{\frac{1}{4}} \text { for any } \theta \in S^{1} .
$$

Proof of Lemma.

Step 1 . We will show $B_{\frac{1}{2}}(0) \subset \Omega$.

Proof of Step 1. Suppose not, so we can select $x \in \partial \Omega \cap B_{\frac{1}{2}}(0)$. Let $\eta_{x}$ be the inward pointing unit normal to $\partial \Omega$ at $x$. By convexity of $\Omega$ we have $\Omega \subset \overline{H\left(x, \eta_{x}\right)}$ and so $B_{1}(0) \cap H\left(x,-\eta_{x}\right) \cap \Omega=\emptyset$ which implies $\left|B_{1}(0) \backslash \Omega\right| \geq\left|B_{1}(0) \cap H\left(x,-\eta_{x}\right)\right|>\frac{1}{8}$ which contradicts that $\left|\Omega \triangle B_{1}\right| \leq \beta$.

Step 2. $a_{\theta} \in B_{1+c \sqrt{\beta}}(0)$.

Proof of Step 2. Suppose not. Since $\Omega$ is convex we have conv $\left(\left\{a_{\theta}\right\} \cup B_{\frac{1}{2}}(0)\right) \subset \Omega$ and

$$
\left|\operatorname{conv}\left(\left\{a_{\theta}\right\} \cup B_{\frac{1}{2}}(0)\right) \backslash B_{1}(0)\right|>c \beta .
$$

Thus we have $\left|\Omega \backslash B_{1}(0)\right|>c \beta$ which contradicts the fact that $\left|\Omega \triangle B_{1}(0)\right|=\beta$.

Step 3. We will show $a_{\theta} \notin B_{1-c \sqrt{\beta}}(0)$.

Proof of Step 3. Suppose $a_{\theta} \in B_{1-c \sqrt{\beta}}(0)$ this implies $\left|B_{1}(0) \backslash H\left(a_{\theta}, \eta_{a_{\theta}}\right)\right| \geq c \beta^{\frac{3}{4}}$ and $\Omega \subset H\left(a_{\theta}, \eta_{a_{\theta}}\right)$ so $\left|B_{1}(0) \backslash \Omega\right| \geq c \beta^{\frac{3}{4}}$ which gives a contradiction.

Proof of Lemma completed. Suppose (4.3) is false, since $\left|a_{\theta}-\theta\right| \leq c \sqrt{\beta}$ we have

$$
\left|B_{1}(0) \backslash H\left(a_{\theta}, \eta_{a_{\theta}}\right)\right| \geq c \beta^{\frac{3}{4}} .
$$

As before this implies $\left|B_{1}(0) \backslash \Omega\right|>c \beta^{\frac{3}{4}}$ which is a contradiction. 
Lemma 4.3. Let $\Omega$ be convex and define $u(x):=d(z, \partial \Omega)$ for any $z \in \Omega$ then function $u$ is concave.

Proof of Lemma. Let $a, b \in \Omega$. Since $\Omega$ is convex $\operatorname{conv}\left(B_{u(a)}(a) \cup B_{u(b)}(b)\right) \subset$ $\Omega$. Now suppose there exists $\lambda \in(0,1)$ such that

$$
u(\lambda a+(1-\lambda) b)<\lambda u(a)+(1-\lambda) u(b)
$$

then as this implies $B_{u(\lambda a+(1-\lambda) b)}(\lambda a+(1-\lambda) b) \subset \operatorname{int}\left(\operatorname{conv}\left(B_{u(a)}(a) \cup B_{u(b)}(b)\right)\right)$ we must be able to find $x \in \partial \Omega$ with $x \in \partial \Omega \cap \operatorname{conv}\left(B_{u(a)}(a) \cup B_{u(b)}(b)\right)$ which is a contradiction.

Lemma 4.4. Let $\beta>0$. Suppose $\Omega$ is a convex set with $\left|\Omega \triangle B_{1}(0)\right| \leq \beta$. Let $u(z)=d(z, \partial \Omega)$. For any $x \in \Omega \backslash B_{\beta^{\frac{1}{8}}}(0)$ for which the approximate derivative $\nabla u$ exists

$$
\left|\nabla u(x)+\frac{x}{|x|}\right| \leq c \beta^{\frac{3}{16}} .
$$

Proof. For any $x \in \Omega \backslash B_{\beta^{\frac{1}{8}}}(0)$ let $b_{x} \in \partial \Omega$ be such that $\left|b_{x}-x\right|=u(x)$.

We begin by showing

$$
\left|b_{x}-\frac{x}{|x|}\right| \leq c \beta^{\frac{3}{16}}
$$

Recall from Lemma $4.2 a_{\frac{x}{|x|}}=\partial \Omega \cap l_{0}^{\frac{x}{|x|}}$. Using (4.2) from Lemma 4.2 and the fact $\left(x-a_{\frac{x}{|x|}}\right)\left|x-a_{\frac{x}{|x|} \mid}\right|^{-1}=\frac{x}{|x|}$ we have

$$
\left|x-b_{x}\right| \leq\left|x-a_{\frac{x}{|x|} \mid} \stackrel{(4.2)}{\leq} 1-\right| x \mid+c \sqrt{\beta} .
$$

Hence

$$
\left|x-b_{x}\right|^{2}=|x|^{2}-2 x \cdot b_{x}+\left|b_{x}\right|^{2} \stackrel{(4.5)}{\leq} 1-2|x|+|x|^{2}+c \sqrt{\beta} .
$$

Therefore

$$
\begin{aligned}
-2 x \cdot b_{x} & \stackrel{(4.6)}{\leq} 1-2|x|+c \sqrt{\beta}-\left|b_{x}\right|^{2} \\
& \stackrel{(4.2)}{\leq}-2|x|+c \sqrt{\beta} .
\end{aligned}
$$

Thus $2|x| \leq 2 x \cdot b_{x}+c \sqrt{\beta}$. Since $|x|>\beta^{\frac{1}{8}}$ we have

$$
1-c \beta^{\frac{3}{8}} \leq 1-c \frac{\beta^{\frac{1}{2}}}{|x|} \leq \frac{x}{|x|} \cdot b_{x} .
$$


Hence

$$
\left|b_{x}-\frac{x}{|x|}\right|^{2}=\left|b_{x}\right|^{2}+1-2 \frac{x}{|x|} \cdot b_{x} \stackrel{(4.7),(4.2)}{\leq} c \beta^{\frac{3}{8}}
$$

which gives

$$
\left|\frac{x}{|x|}-b_{x}\right| \leq c \beta^{\frac{3}{16}}
$$

Let $\theta_{x}=\frac{b_{x}}{\left|b_{x}\right|}$ so using Lemma $4.2\left|\eta_{b_{x}}+\frac{b_{x}}{\left|b_{x}\right|}\right|=\left|\eta_{a_{\theta_{x}}}+\theta_{x}\right| \stackrel{(4.3)}{\leq} c \beta^{\frac{1}{4}}$ and by (4.2) this easily implies

$$
\left|\eta_{b_{x}}+b_{x}\right| \leq c \beta^{\frac{1}{4}}
$$

Now since $\nabla u(x)=\frac{x-b_{x}}{\left|x-b_{x}\right|}=\eta_{b_{x}}$ and so

$$
\left|\nabla u(x)+\frac{x}{|x|}\right| \leq\left|\eta_{b_{x}}+b_{x}\right|+\left|\frac{x}{|x|}-b_{x}\right| \stackrel{(4.8),(4.9)}{\leq} c \beta^{\frac{3}{16}}
$$

thus we have established (4.4).

Lemma 4.5. Let $\Omega$ be a convex set and $\left|\Omega \triangle B_{1}(0)\right| \leq \beta$. Define $u(x)=d(x, \partial \Omega)$. Note that since $u$ is convex $\nabla u$ is $B V$. Let $V(\nabla u, \cdot)$ denotes the total variation of the measure $\nabla u$. Firstly we have

$$
V\left(\nabla u, \Omega \backslash \overline{B_{3 \beta^{\frac{1}{8}}}(0)}\right) \leq 16 \pi .
$$

Secondly for any $\varepsilon \in\left(0, \beta^{\frac{1}{2}}\right]$ and for any $x \in \Omega \backslash\left(N_{2 \varepsilon}(\partial \Omega) \cup B_{4 \beta^{\frac{1}{8}}}(0)\right)$ we have

$$
V\left(\nabla u, B_{\varepsilon}(x)\right) \leq c \beta^{\frac{3}{16}} \varepsilon .
$$

Proof. Let $\tau \in\left(0, \frac{\varepsilon}{20}\right)$ be some small number.

For any $x \in \Omega \backslash\left(\overline{N_{4 \tau}(\partial \Omega) \cup B_{\frac{3}{2} \beta^{\frac{1}{8}}}(0)}\right)=: \Pi_{\tau}$. Let $w_{\tau}(x)=u * \rho_{\tau}(x)$ and $v^{\tau}=$ $\frac{\nabla w_{\tau}}{\left|\nabla w_{\tau}\right|}$. Note from Lemma 4.4 for any $x \in \Pi_{\tau}$

$$
\begin{aligned}
\left|\nabla w_{\tau}(x)+\frac{x}{|x|}\right| & =\left|\int\left(\nabla u(x-z)+\frac{x}{|x|}\right) \rho_{\tau}(z) d z\right| \\
& \leq \int\left|\left(\nabla u(x-z)+\frac{x-z}{|x-z|}\right) \rho_{\tau}(z)\right| d z \\
& +\int\left|\frac{x-z}{|x-z|}-\frac{x}{|x|}\right| \rho_{\tau}(z) d z \\
& \stackrel{(4.4)}{\leq} c \sup _{z \in B_{2 \tau}(0)}\left|\frac{x-z}{|x-z|}-\frac{x}{|x|}\right|+c \beta^{\frac{3}{16}} \\
& \leq c \beta^{\frac{3}{16}} .
\end{aligned}
$$


From this it is easy to conclude that

$$
\left.\| w_{\tau}-\operatorname{dist}\left(\cdot, \partial B_{1}(0)\right)\right) \|_{L^{\infty}\left(\Pi_{\tau}\right)} \leq c \beta^{\frac{3}{16}}
$$

Step 1 . Let $\tau_{0}>0$ be a very small number. We will show

$$
\lim _{\tau \rightarrow 0}\left\|v^{\tau}-\nabla u\right\|_{L^{1}\left(\Pi_{\tau_{0}}\right)}=0 .
$$

Proof of Step 1. Now

$$
\begin{aligned}
\int_{\Pi_{\tau_{0}}}|1-| \nabla w_{\tau}|| d z & =\int_{\Pi_{\tau_{0}}}|| \nabla u|-| \nabla w_{\tau}|| d z \\
& \leq \int_{\Pi_{\tau_{0}}}\left|\nabla u-\nabla w_{\tau}\right| d z \rightarrow 0 \text { as } \tau \rightarrow 0 .
\end{aligned}
$$

Now from (4.12) we have

$$
\left|\nabla w_{\tau}(x)\right| \geq \frac{1}{2} \text { for any } x \in \Pi_{\tau_{0}}, \tau \in\left(0, \tau_{0}\right) .
$$

So

$$
\begin{aligned}
&\left\|\frac{\nabla w_{\tau}}{\left|\nabla w_{\tau}\right|}-\nabla w_{\tau}\right\|_{L^{1}\left(\Pi_{\tau_{0}}\right)}=\left\|\nabla w_{\tau}\left(\frac{1}{\left|\nabla w_{\tau}\right|}-1\right)\right\|_{L^{1}\left(\Pi_{\tau_{0}}\right)} \\
& \stackrel{(4.15),(4.16)}{\leq} 2\left\|1-\left|\nabla w_{\tau}\right|\right\|_{L^{1}\left(\Pi_{\tau_{0}}\right)} \rightarrow 0 \text { as } \tau \rightarrow 0 .
\end{aligned}
$$

Since $\left\|\nabla w_{\tau}-\nabla u\right\|_{L^{1}\left(\Pi_{\tau_{0}}\right)} \rightarrow 0$ as $\tau \rightarrow 0$ putting this together with (4.17) gives (4.14).

Step 2. We will show that for any $G \Subset \Omega \backslash \overline{B_{\frac{3}{2} \beta^{\frac{1}{8}}}(0)}$

$$
V(\nabla u, G) \leq 2|\operatorname{div}(\nabla u)|(G)
$$

and

$$
|\operatorname{div}(\nabla u)|(G) \leq \liminf _{\tau \rightarrow 0} \int_{G}\left|v_{1,1}^{\tau}+v_{2,2}^{\tau}\right| d z,
$$

where $|\operatorname{div}(\nabla u)|$ denotes the total variation of measure $\operatorname{div}(\nabla u)$.

Proof of Step 2. We can find $\tau_{0}>0$ such that $G \subset \Pi_{\tau_{0}}$. Now from [3] $\nabla u \in S B V_{\text {loc }}$ so in particular $\operatorname{div}(\nabla u)$ is a signed measure defined by

$$
\int \operatorname{div}(\nabla u) \phi d z=\int \phi_{, 1} u, 1+\phi_{, 2} u, 2 d z \text { for all } \phi \in C_{c}^{\infty}(\Omega) .
$$


So for $\phi \in C_{c}^{\infty}(\Omega)$ we have

$$
\begin{aligned}
\int \operatorname{div}(\nabla u) \phi d z \stackrel{(4.20),(4.14)}{=} & \lim _{\tau \rightarrow 0} \int \phi_{, 1} v_{1}^{\tau}+\phi_{, 2} v_{2}^{\tau} d z \\
& =\lim _{\tau \rightarrow 0} \int\left(v_{1,1}^{\tau}+v_{2,2}^{\tau}\right) \phi d z
\end{aligned}
$$

Now given open set $G \subset \Pi_{\tau_{0}}$ if $\phi \in C_{c}^{\infty}(G)$ then

$$
\begin{aligned}
\left|\int \operatorname{div}(\nabla u) \phi d z\right| & =\left|\lim _{\tau \rightarrow 0} \int\left(v_{1,1}^{\tau}+v_{2,2}^{\tau}\right) \phi d z\right| \\
& \leq\|\phi\|_{L^{\infty}\left(\Pi_{\tau_{0}}\right)} \int_{G}\left|v_{1,1}^{\tau}+v_{2,2}^{\tau}\right| d z .
\end{aligned}
$$

So this in particular by Proposition 1.47 [4] implies (4.19).

Now since $\nabla u \in S B V_{\text {loc }}(\Omega)$ we know by Theorem 3.78 [4] that there exists a rectifiable set $J_{\nabla u} \subset S_{\nabla u}$ (where $S_{\nabla u}$ denotes the set of approximate jump points of $\nabla u)$ with $H^{n-1}\left(S_{\nabla u} \backslash J_{\nabla u}\right)=0$ and $D \nabla u\left\lfloor J_{\nabla u}=\left(\nabla u^{+}-\nabla u^{-}\right) \otimes\right.$ $v H^{n-1}\left\lfloor J_{\nabla u}\right.$ where $v(x)$ is the normal to the approximate tangent of the rectifiable set $J_{\nabla u}$ at point $x$. Following [4] Definition 3.67 we assume that the triple $\left(\nabla u^{+}(x), \nabla u^{-}(x), v(x)\right)$ satisfies (3.69) of [4]. By Theorem 3.94 [4] we have that $\left(\nabla u^{+}(x)-\nabla u^{-}(x)\right) \otimes v(x)$ is a rank-1 matrix for $|D \nabla u|$ a.e. $x \in J_{\nabla u}$. Now $D \nabla u$ is a matrix valued measure and indeed letting $\partial_{i} u, j$ denote the individual 'component' measures, just from the definition we know that $\partial_{i} u_{, j}=\partial_{j} u_{, i}$ so $D \nabla u$ is a symmetric matrix valued measure. Specifically by differentiation of measures (see Theorem 2.2 [4] $M(x):=\lim _{r \rightarrow 0} \frac{D \nabla u\left(B_{r}(x)\right)}{|D \nabla u|\left(B_{r}(x)\right)}$ exists for $|D \nabla u|$ a.e. $x$ and $M(x)$ will be a symmetric $2 \times 2$ matrix. So for $H^{n-1}$ a.e. $x \in J_{\nabla u},\left(\nabla u^{+}(x)-\nabla u^{-}(x)\right) \otimes v(x)$ is a symmetric rank-1 matrix, this is easily seen to imply $\frac{\nabla u^{+}(x)-\nabla u^{-}(x)}{\left|\nabla u^{+}(x)-\nabla u^{-}(x)\right|}=v(x)$. So $\left(\nabla u^{+}(x)-\nabla u^{-}(x)\right) \otimes v(x)=\left|\nabla u^{+}(x)-\nabla u^{-}(x)\right| v(x) \otimes v(x)$. Thus we can decompose $D(\nabla u)$ into absolutely continuous and singular parts we have

$$
\begin{aligned}
D(\nabla u)(S)= & \int_{S} D(\nabla u) d x+\int_{S \cap J_{\nabla u}}\left|\nabla u^{+}-\nabla u^{-}\right| v(x) \otimes v(x) d H^{1} \\
& \text { for any set } S \subset \mathbb{R}^{n} .
\end{aligned}
$$

Obviously this is a matrix valued Radon measure and the signed Radon measure $\Delta u$ is given by the sum of diagonal elements of the matrix defined by (4.21) and so is given by

$$
\begin{aligned}
\Delta u(S) & =\int_{S} \operatorname{div}_{a}(\nabla u) d x+\int_{S \cap J_{\nabla u}}\left|\nabla u^{+}-\nabla u^{-}\right| v \cdot v d H^{1} \\
& =\int_{S} \operatorname{div}_{a}(\nabla u) d x+\int_{S \cap J_{\nabla u}}\left|\nabla u^{+}-\nabla u^{-}\right| d H^{1} \text { for any } S \subset \mathbb{R}^{n} .
\end{aligned}
$$


Now recall $|\nabla u(x)|=1$ for a.e. $x \in \Omega$. So by Volpert chain rule (see Theorem 3.94 [4]) we have that the function $x \rightarrow|\nabla u(x)|^{2}$ is BV and the standard chain rule holds so

$$
\begin{aligned}
& u_{, 11}(x) u_{, 1}(x)+u_{, 12}(x) u_{, 2}(x)=0 \text { and } \\
& u_{, 12}(x) u_{, 1}(x)+u_{, 22}(x) u_{, 2}(x)=0 \text { for a.e. } x \in \Omega .
\end{aligned}
$$

Since $u, 21=u_{, 12}$ we have

$$
\left(\begin{array}{ll}
u, 11 & u_{, 12} \\
u_{, 21} & u_{, 22}
\end{array}\right)\left(\begin{array}{l}
u, 1 \\
u_{, 2}
\end{array}\right) \stackrel{(4.22)}{=}\left(\begin{array}{l}
0 \\
0
\end{array}\right) \text { and }\left(\begin{array}{ll}
u, 11 & u_{, 12} \\
u_{, 21} & u_{, 22}
\end{array}\right)\left(\begin{array}{c}
-u_{, 2} \\
u_{, 1}
\end{array}\right) \stackrel{(4.22)}{=}\left(u_{, 11}+u_{, 22}\right)\left(\begin{array}{c}
-u_{, 2} \\
u, 1
\end{array}\right) \text {. }
$$

Letting $\|\cdot\|$ denote the operator norm of a matrix, since $\left(\begin{array}{cc}u_{, 1} & -u_{, 2} \\ u_{, 2} & u_{, 1}\end{array}\right) \in O(2)$ we have

$$
\begin{aligned}
\left\|\left(\begin{array}{ll}
u_{, 11} & u_{, 12} \\
u_{, 21} & u_{, 22}
\end{array}\right)\right\| & =\left\|\left(\begin{array}{cc}
u, 11 & u_{, 12} \\
u, 21 & u_{, 22}
\end{array}\right)\left(\begin{array}{cc}
u_{, 1} & -u_{, 2} \\
u_{, 2} & u_{, 1}
\end{array}\right)\right\| \\
& =\left\|\left(\begin{array}{cc}
0 & -\left(u_{, 11}+u_{, 22}\right) u_{, 2} \\
0 & \left(u_{, 11}+u_{, 22}\right) u_{, 1}
\end{array}\right)\right\| \\
& \leq 2\left|u_{, 11}+u_{, 22}\right| .
\end{aligned}
$$

Hence

$$
\left|D_{a}(\nabla u(x))\right| \leq 2\left|\operatorname{div}_{a}(\nabla u(x))\right| \text { for a.e. } x \in \Omega \text {. }
$$

Thus

$$
\begin{aligned}
V(\nabla u, G) & =\int_{G}\left|D_{a}(\nabla u)\right| d z+\int_{G \cap J_{\nabla u}}\left|\nabla u^{+}-\nabla u^{-}\right| d H^{1} \\
& \leq 2 \int_{G}\left|\operatorname{div}_{a}(\nabla u)\right| d z+\int_{G \cap J_{\nabla u}}\left|\nabla u^{+}-\nabla u^{-}\right| d H^{1} \\
& \leq 2|\operatorname{div}(\nabla u)|(G),
\end{aligned}
$$

thus establishing (4.18).

Step 3. We will show that for any $t \in\left(8 \tau, 1-2 \beta^{\frac{1}{8}}\right)$

$$
\int_{w_{\tau}^{-1}(t)}\left|v_{1,1}^{\tau}(z)+v_{2,2}^{\tau}(z)\right| d H^{1} z \leq 2 \pi
$$

Proof of Step 3. We define the 'angle' function by

$$
A(x):=\left\{\begin{array}{ll}
\arccos \left(\frac{x_{1}}{|x|}\right) & \text { for } x_{2} \geq 0 \\
2 \pi-\arccos \left(\frac{x_{1}}{|x|}\right) & \text { for } x_{2}<0
\end{array} .\right.
$$


Note that $A$ is smooth expect at the half line $\left\{\left(x_{1}, x_{2}\right): x_{2}=0, x_{1}>0\right\}$. For $x \in \Pi_{\tau}$ we have $\left|v^{\tau}(x)\right|^{2}=1$, so as before

$$
\partial_{1}\left(\left|v^{\tau}(x)\right|^{2}\right)=v_{1}^{\tau}(x) v_{1,1}^{\tau}(x)+v_{2}^{\tau}(x) v_{2,1}^{\tau}(x)=0 .
$$

Since $u$ is 1-Lipschitz,

$$
\left\|w_{\tau}-u\right\|_{L^{\infty}\left(\Pi_{\tau}\right)} \leq 2 \tau
$$

and so from this and (4.13) we have that $w_{\tau}^{-1}(t) \subset \Pi_{\tau}$ for any $t \in\left(8 \tau, 1-2 \beta^{\frac{1}{8}}\right)$. Hence by (4.12) $v^{\tau}$ is well defined along this level set. We also know that for any $x \in w_{\tau}^{-1}(t)$ the tangent to curve $w_{\tau}^{-1}(t)$ is given by $\left(\begin{array}{c}-v_{2}^{\tau}(x) \\ v_{1}^{\tau}(x)\end{array}\right)$. Note that $w_{\tau}^{-1}(t)$ is the boundary of a smooth convex set so there exists a point $x_{t} \in w_{\tau}^{-1}(t)$ such that $A\left(\begin{array}{c}-v_{2}^{\tau}\left(x_{t}\right) \\ v_{1}^{\tau}\left(x_{t}\right)\end{array}\right)=0$. There must also exist $y_{t} \in w_{\tau}^{-1}(t)$ such that

$$
A\left(\begin{array}{c}
-v_{2}^{\tau}\left(y_{t}\right) \\
v_{1}^{\tau}\left(y_{t}\right)
\end{array}\right)=\pi
$$

Let $\Phi^{t}:\left[0, H^{1}\left(w_{\tau}^{-1}(t)\right)\right) \rightarrow w_{\tau}^{-1}(t)$ denote the clockwise parameterization of $w_{\tau}^{-1}(t)$ by arc-length with $\Phi^{t}(0)=x_{t}$. So $\dot{\Phi}^{t}(s)=\left(\begin{array}{c}-v_{2}^{\tau}\left(\Phi^{t}(s)\right) \\ v_{1}^{\tau}\left(\Phi^{t}(s)\right)\end{array}\right)$. Define $\Theta_{t}$ : $\left[0, H^{1}\left(w_{\tau}^{-1}(t)\right)\right) \rightarrow \mathbb{R}$ by $\Theta_{t}(s)=A\left(\dot{\Phi}^{t}(s)\right)$. Now select $s \in\left(0, H^{1}\left(w_{\tau}^{-1}(t)\right)\right)$, suppose $v_{1}^{\tau}\left(\Phi^{t}(s)\right)>0$, then

$$
\begin{aligned}
\dot{\Theta}_{t}(s) & =\arccos \left(-v_{2}^{\tau}\left(\Phi^{t}(s)\right)\right) \frac{\partial}{\partial t}\left(-v_{2}^{\tau}\left(\Phi^{t}(s)\right)\right) \\
& =\arccos \left(-v_{2}^{\tau}\left(\Phi^{t}(s)\right)\right)\left(-v_{2,1}^{\tau}\left(\Phi^{t}(s)\right) \dot{\Phi}_{1}^{t}(t)-v_{2,2}^{\tau}\left(\Phi^{t}(s)\right) \dot{\Phi}_{2}^{t}(t)\right) \\
& =\arccos \left(-v_{2}^{\tau}\left(\Phi^{t}(s)\right)\right)\left(v_{2,1}^{\tau}\left(\Phi^{t}(s)\right) v_{2}^{\tau}\left(\Phi^{t}(s)\right)-v_{2,2}^{\tau}\left(\Phi^{t}(s)\right) v_{1}^{\tau}\left(\Phi^{t}(s)\right)\right) \\
& \stackrel{(4.24)}{=} \arccos \left(-v_{2}^{\tau}\left(\Phi^{t}(s)\right)\right)\left(-v_{1,1}^{\tau}\left(\Phi^{t}(s)\right) v_{1}^{\tau}\left(\Phi^{t}(s)\right)-v_{2,2}^{\tau}\left(\Phi^{t}(s)\right) v_{1}^{\tau}\left(\Phi^{t}(s)\right)\right) \\
& =-\arccos \left(-v_{2}^{\tau}\left(\Phi^{t}(s)\right)\right) v_{1}^{\tau}\left(\Phi^{t}(s)\right)\left(v_{1,1}^{\tau}\left(\Phi^{t}(s)\right)+v_{2,2}^{\tau}\left(\Phi^{t}(s)\right)\right) .
\end{aligned}
$$

Now for any $w \in(-1,1), \arccos (w)=-(\sin (\arccos (w)))^{-1}$ so

$$
\dot{\Theta}_{t}(t)=\frac{v_{1}^{\tau}\left(\Phi^{t}(s)\right)}{\sin \left(\arccos \left(-v_{2}^{\tau}\left(\Phi^{t}(s)\right)\right)\right)}\left(v_{1,1}^{\tau}\left(\Phi^{t}(s)\right)+v_{2,2}^{\tau}\left(\Phi^{t}(s)\right)\right) \text {. }
$$

Recall $\left|\left(\begin{array}{c}-v_{2}^{\tau}\left(\Phi^{t}(s)\right) \\ v_{1}^{\tau}\left(\Phi^{t}(s)\right)\end{array}\right)\right|=1$ and we supposed $v_{1}^{\tau}\left(\Phi^{t}(s)\right)>0$, so

$$
\begin{array}{rlr}
v_{1}^{\tau}\left(\Phi^{t}(s)\right) & = & \sqrt{1-\left(v_{2}^{\tau}\left(\Phi^{t}(s)\right)\right)^{2}} \\
& = & \sqrt{1-\left(\cos \left(\arccos \left(-v_{2}^{\tau}\left(\Phi^{t}(s)\right)\right)\right)\right)^{2}} \\
& = & \sin \left(\arccos \left(-v_{2}^{\tau}\left(\Phi^{t}(s)\right)\right)\right) .
\end{array}
$$


Thus from (4.27)

$$
\begin{aligned}
\dot{\Theta}_{t}(s)= & \left(v_{1,1}^{\tau}\left(\Phi^{t}(s)\right)+v_{2,2}^{\tau}\left(\Phi^{t}(s)\right)\right) \\
& \text { for any } s \in\left(0, H^{1}\left(w_{\tau}^{-1}(t)\right)\right) \text { with } v_{1}^{\tau}\left(\Phi^{t}(s)\right)>0
\end{aligned}
$$

Suppose we have $s \in\left(0, H^{1}\left(w_{\tau}^{-1}(t)\right)\right)$ with $v_{1}^{\tau}\left(\Phi^{t}(s)\right)<0$, then in the same way as (4.28) we have

$$
\begin{aligned}
v_{1}^{\tau}\left(\Phi^{t}(s)\right) & =-\sqrt{1-\left(\cos \left(\arccos \left(-v_{2}^{\tau}\left(\Phi^{t}(s)\right)\right)\right)\right)^{2}} \\
& =-\sin \left(\arccos \left(-v_{2}^{\tau}\left(\Phi^{t}(s)\right)\right)\right) .
\end{aligned}
$$

And since $v_{1}^{\tau}\left(\Phi^{t}(s)\right)<0$, by definition of $A$ (see (4.23)) arguing as in (4.27) we have

$$
\begin{aligned}
\dot{\Theta}_{t}(s) & =\frac{-v_{1}^{\tau}\left(\Phi^{t}(s)\right)}{\sin \left(\arccos \left(-v_{2}^{\tau}\left(\Phi^{t}(s)\right)\right)\right)}\left(v_{1,1}^{\tau}\left(\Phi^{t}(s)\right)+v_{2,2}^{\tau}\left(\Phi^{t}(s)\right)\right) \\
\stackrel{(4.30)}{=} & v_{1,1}^{\tau}\left(\Phi^{t}(s)\right)+v_{2,2}^{\tau}\left(\Phi^{t}(s)\right) \text { for } s \in\left(0, H^{1}\left(w_{\tau}^{-1}(t)\right)\right) \text { with } v_{1}^{\tau}\left(\Phi^{t}(s)\right)<0 .
\end{aligned}
$$

Without loss of generality we can assume $\left|\left\{s \in\left[0, H^{1}\left(w_{\tau}^{-1}(t)\right)\right]: v_{1}^{\tau}\left(\Phi^{t}(s)\right)=0\right\}\right|=$ 0 . Thus by continuity of $\dot{\Theta}_{t}(\cdot), v_{1,1}^{\tau}\left(\Phi^{t}(\cdot)\right)$ and $v_{2,2}^{\tau}\left(\Phi^{t}(\cdot)\right)$ we have

$$
\dot{\Theta}_{t}(s)=v_{1,1}^{\tau}\left(\Phi^{t}(s)\right)+v_{2,2}^{\tau}\left(\Phi^{t}(s)\right) \text { for } s \in\left[0, H^{1}\left(w_{\tau}^{-1}(t)\right)\right) .
$$

Now since $u$ is concave, $w_{\tau}$ is concave and so the set $w_{\tau}^{-1}([t, \infty))$ is a convex set, hence

$$
v_{1,1}^{\tau}\left(\Phi^{t}(s)\right)+v_{2,2}^{\tau}\left(\Phi^{t}(s)\right)=\dot{\Theta}_{t}(s) \geq 0 \text { for any } s \in\left[0, H^{1}\left(w_{\tau}^{-1}(t)\right)\right) .
$$

Therefore

$$
\int_{w_{\tau}^{-1}(t)}\left|v_{1,1}^{\tau}(z)+v_{2,2}^{\tau}(z)\right| d H^{1} z=\int_{0}^{H^{1}\left(w_{\tau}^{-1}(t)\right)} \dot{\Theta}_{t}(s) d s \leq 2 \pi .
$$

Step 4. Let $x \in \Pi_{\tau} \backslash N_{2 \varepsilon}(\partial \Omega)$ and define

$$
\begin{aligned}
& t_{1}=\inf \left\{s \in \mathbb{R}: w_{\tau}^{-1}(s) \cap B_{\varepsilon}(x) \neq \emptyset\right\} \text { and } \\
& t_{2}=\sup \left\{s \in \mathbb{R}: w_{\tau}^{-1}(s) \cap B_{\varepsilon}(x) \neq \emptyset\right\} .
\end{aligned}
$$

Recall $y_{t} \in w_{\tau}^{-1}(t)$ was chosen so that (4.26) holds true, let $\pi_{t}:=\left(\Phi^{t}\right)^{-1}\left(y_{t}\right)$. We have for any $t \in\left(t_{1}, t_{2}\right)$

$$
\sup \left\{\left|\Theta_{t}\left(s_{1}\right)-\Theta_{t}\left(s_{2}\right)\right|: s_{1}, s_{2} \in\left(\Phi^{t}\right)^{-1}\left(w_{\tau}^{-1}(t) \cap B_{\varepsilon}(x)\right) \cap\left[0, \pi_{t}\right]\right\} \leq c \beta^{\frac{3}{16}}
$$


and

$$
\begin{aligned}
& \sup \left\{\left|\Theta_{t}\left(s_{1}\right)-\Theta_{t}\left(s_{2}\right)\right|: s_{1}, s_{2} \in\left(\Phi^{t}\right)^{-1}\left(w_{\tau}^{-1}(t) \cap B_{\varepsilon}(x)\right) \cap\left[\pi_{t}, H^{1}\left(w_{\tau}^{-1}(t)\right)\right)\right\} \\
& \leq c \beta^{\frac{3}{16}}
\end{aligned}
$$

Proof of Step 4. Let $s_{1}, s_{2} \in\left[0, \pi_{t}\right]$ such that $\Phi^{t}\left(s_{1}\right), \Phi^{t}\left(s_{2}\right) \in B_{\varepsilon}(x)$, since $\Phi^{t}$ is parameterization of $w_{\tau}^{-1}(t)$ by arclength $\dot{\Phi}^{t}(s)$ is the unit tangent to $w_{\tau}^{-1}(t)$ at $\Phi^{t}(s)$. Thus

$$
R\left(\frac{\nabla w_{\tau}\left(\Phi^{t}\left(s_{i}\right)\right)}{\left|\nabla w_{\tau}\left(\Phi^{t}\left(s_{i}\right)\right)\right|}\right)=\dot{\Phi}^{t}\left(s_{i}\right) \text { for } i=1,2
$$

However by Lemma 4.4 (recalling the fact that $\left|\Phi^{t}\left(s_{1}\right)\right|>\frac{3 \beta^{\frac{1}{8}}}{2}$ and $\left|\Phi^{t}\left(s_{2}\right)\right|>\frac{3 \beta^{\frac{1}{8}}}{2}$ in order to apply the lemma)

$$
\begin{aligned}
& \left|\nabla w_{\tau}\left(\Phi^{t}\left(s_{1}\right)\right)-\nabla w_{\tau}\left(\Phi^{t}\left(s_{2}\right)\right)\right| \\
= & \left|\int\left(\nabla u\left(\Phi^{t}\left(s_{1}\right)-z\right)-\nabla u\left(\Phi^{t}\left(s_{2}\right)-z\right)\right) \rho_{\tau}(z) d z\right| \\
\stackrel{(4.4)}{\leq} & c \int_{B_{\tau}(0)}\left|\frac{\Phi^{t}\left(s_{1}\right)-z}{\left|\Phi^{t}\left(s_{1}\right)-z\right|}-\frac{\Phi^{t}\left(s_{2}\right)-z}{\left|\Phi^{t}\left(s_{2}\right)-z\right|}\right| \rho_{\tau}(z) d z+c \beta^{\frac{3}{16}} .
\end{aligned}
$$

Note $z \in B_{\tau}(0) \subset B_{\frac{\beta^{\frac{1}{2}}}{20}}(0)$ so as $\left|\Phi^{t}\left(s_{1}\right)\right|>\frac{3 \beta^{\frac{1}{8}}}{2}$ we have $\left|\Phi^{t}\left(s_{1}\right)-z\right| \geq\left|\Phi^{t}\left(s_{1}\right)\right|-$ $|z| \geq \beta^{\frac{1}{8}}$. Recall the elementary inequality inequality

$$
\left|\frac{z}{|z|}-\frac{y}{|y|}\right| \leq 2|z-y| \text { for any } z, y \text { with }|z| \geq 1,|y| \geq 1 \text {. }
$$

So in particular we have

$$
\| \frac{\Phi^{t}\left(s_{1}\right)-z}{\left|\Phi^{t}\left(s_{1}\right)-z\right|}-\frac{\Phi^{t}\left(s_{2}\right)-z}{\left|\Phi^{t}\left(s_{2}\right)-z\right|}|| \leq \frac{2}{\beta^{\frac{1}{8}}}\left|\Phi^{t}\left(s_{1}\right)-\Phi^{t}\left(s_{2}\right)\right| \leq 2 \beta^{\frac{3}{8}}
$$

Thus with (4.35) this gives

$$
\left|\nabla w_{\tau}\left(\Phi^{t}\left(s_{1}\right)\right)-\nabla w_{\tau}\left(\Phi^{t}\left(s_{2}\right)\right)\right| \leq c \beta^{\frac{3}{16}} .
$$

As a consequence of (4.12) we know

$$
|| \nabla w_{\tau}(x)|-1| \leq c \beta^{\frac{3}{16}} \text { for any } x \in \Pi_{\tau}
$$


SO

$$
\begin{aligned}
\left|\dot{\Phi}\left(s_{1}\right)-\dot{\Phi}\left(s_{2}\right)\right| & \stackrel{(4.36)}{\leq}\left|R\left(\frac{\nabla w_{\tau}\left(\Phi^{t}\left(s_{1}\right)\right)}{\left|\nabla w_{\tau}\left(\Phi^{t}\left(s_{1}\right)\right)\right|}\right)-R\left(\nabla w_{\tau}\left(\Phi^{t}\left(s_{1}\right)\right)\right)\right| \\
& +\left|R\left(\frac{\nabla w_{\tau}\left(\Phi^{t}\left(s_{2}\right)\right)}{\left|\nabla w_{\tau}\left(\Phi^{t}\left(s_{2}\right)\right)\right|}\right)-R\left(\nabla w_{\tau}\left(\Phi^{t}\left(s_{2}\right)\right)\right)\right|+c \beta^{\frac{3}{16}} \\
& \stackrel{(4.37)}{\leq} c \beta^{\frac{3}{16}} .
\end{aligned}
$$

Now as $s_{1}, s_{2} \in\left[0, \pi_{t}\right]$, since $w_{\tau}^{-1}(t)$ is the boundary of a convex set so we know $\dot{\Phi}^{t}\left(s_{1}\right), \dot{\Phi}^{t}\left(s_{2}\right) \in\left\{v \in S^{1}: v \cdot e_{2} \leq 0\right\}$. Now, as $A$ is Lipschitz on $\left\{v \in S^{1}: v \cdot e_{2} \leq 0\right\}$,

$$
\left|\Theta_{t}\left(s_{1}\right)-\Theta_{t}\left(s_{2}\right)\right|=\left|A\left(\dot{\Phi}^{t}\left(s_{1}\right)\right)-A\left(\dot{\Phi}^{t}\left(s_{2}\right)\right)\right| \stackrel{(4.38)}{\leq} c \beta^{\frac{3}{16}}
$$

and so (4.33) is established. Inequality (4.34) follows in exactly the same way.

Step 5. We will show

$$
V\left(\nabla u, B_{\varepsilon}(x)\right) \leq c \varepsilon \beta^{\frac{3}{16}} \text { for all } x \in \Omega \backslash\left(N_{2 \varepsilon}(\partial \Omega) \cup B_{4 \beta^{\frac{1}{4}}}(0)\right)
$$

Proof of Step 5. Let $x \in \Omega \backslash\left(N_{2 \varepsilon}(\partial \Omega) \cup B_{4 \beta^{\frac{1}{4}}}(0)\right)$. Let $t \in\left(t_{1}, t_{2}\right)$. The most non-trivial case is where

$$
\left\{s \in\left[0, \pi_{t}\right]: \Phi^{t}(s) \in B_{\varepsilon}(x)\right\} \neq \emptyset \text { and }\left\{s \in\left[\pi_{t}, H^{1}\left(w_{\tau}^{-1}(t)\right)\right]: \Phi^{t}(s) \in B_{\varepsilon}(x)\right\} \neq \emptyset .
$$

When either of these sets is empty the proof follows in a very similar way.

Let $s_{1}^{t}=\inf \left\{s \in\left[0, \pi_{t}\right]: \Phi^{t}(s) \in B_{\varepsilon}(x)\right\}, s_{2}^{t}=\sup \left\{s \in\left[0, \pi_{t}\right]: \Phi^{t}(s) \in B_{\varepsilon}(x)\right\}$. So $\left[s_{1}^{t}, s_{2}^{t}\right]=\left\{s \in\left[0, \pi_{t}\right]: \Phi^{t}(s) \in B_{\varepsilon}(x)\right\}$. Now

$$
\begin{gathered}
\int_{\left[s_{1}^{t}, s_{2}^{t}\right]}\left|v_{1,1}^{\tau}\left(\Phi^{t}(s)\right)+v_{2,2}^{\tau}\left(\Phi_{t}(s)\right)\right| d s \stackrel{(4.31)}{=} \int_{\left[s_{1}^{t}, s_{2}^{t}\right]} \dot{\Theta}_{t}(s) d s \\
\stackrel{(4.33)}{\leq} c \beta^{\frac{3}{16}}
\end{gathered}
$$

In the same way we let

$$
\begin{aligned}
& r_{1}^{t}=\inf \left\{s \in\left[\pi_{t}, H^{1}\left(w_{\tau}^{-1}(t)\right)\right]: \Phi^{t}(s) \in B_{\varepsilon}(x)\right\} \\
& r_{2}^{t}=\sup \left\{s \in\left[\pi_{t}, H^{1}\left(w_{\tau}^{-1}(t)\right)\right]: \Phi^{t}(s) \in B_{\varepsilon}(x)\right\}
\end{aligned}
$$


then

$$
\int_{\left[r_{1}^{t}, r_{2}^{t}\right]}\left|v_{1,1}^{\tau}\left(\Phi^{t}(s)\right)+v_{2,2}^{\tau}\left(\dot{\Phi}_{t}(s)\right)\right| d s \leq c \beta^{\frac{3}{16}}
$$

Thus

$$
\begin{aligned}
\int_{B_{\varepsilon}(x)} \mid & v_{1,1}^{\tau}(z)+v_{2,2}^{\tau}(z)|| \nabla w_{\tau}(z) \mid d z \\
& =\int_{t_{1}}^{t_{2}} \int_{w_{\tau}^{-1}(t)}\left|v_{1,1}^{\tau}(z)+v_{2,2}^{\tau}(z)\right| d H^{1} z d t \\
& =\int_{t_{1}}^{t_{2}} \int_{\left[s_{1}^{t}, s_{2}^{t}\right] \cup\left[r_{1}^{t}, r_{2}^{t}\right]}\left|v_{1,1}^{\tau}\left(\Phi^{t}(s)\right)+v_{1,1}^{\tau}\left(\Phi^{t}(s)\right)\right| d s d t \\
& \leq t_{(4.40),(4.41)}^{\leq} c\left|t_{1}-t_{2}\right| \beta^{\frac{3}{16}} .
\end{aligned}
$$

By using (4.12) and recalling the definition (4.32) of Step 2 we must have $\left|t_{1}-t_{2}\right| \leq$ $c \varepsilon$. Also from (4.12) we know $\left|\nabla w_{\tau}(z)\right| \geq 1-c \beta^{\frac{3}{16}}$ for all $z \in B_{\varepsilon}(x)$, so putting these things together we have

$$
\int_{B_{\varepsilon}(x)}\left|v_{1,1}^{\tau}(z)+v_{2,2}^{\tau}(z)\right| d z \leq c \varepsilon \beta^{\frac{3}{16}} \text { for all } x \in \Omega \backslash\left(N_{2 \varepsilon}(\partial \Omega) \cup B_{4 \beta^{\frac{1}{8}}}(0)\right) \text {. }
$$

So for any $x \in \Omega \backslash\left(N_{2 \varepsilon}(\partial \Omega) \cup B_{4 \beta^{\frac{1}{8}}}(0)\right)$ we know $B_{\varepsilon}(x) \subset \Pi_{\frac{\varepsilon}{4}}$ so by Step 2

$$
\begin{aligned}
& V\left(\nabla u, B_{\varepsilon}(x)\right) \stackrel{(4.18)}{\leq} 2|\operatorname{div}(\nabla u)|\left(B_{\varepsilon}(x)\right) \\
& \stackrel{(4.19)}{\leq} 2 \liminf _{\tau \rightarrow 0} \int_{B_{\varepsilon}(x)}\left|v_{1,1}^{\tau}+v_{2,2}^{\tau}\right| d z \\
& \leq c \varepsilon \beta^{\frac{3}{16}},
\end{aligned}
$$

and hence we have established (4.39).

Proof of Lemma completed. Note that by (4.13) and (4.25) we have

$$
\Pi_{16 \tau} \backslash \overline{B_{3 \beta^{\frac{1}{8}}}(0)} \subset w_{\tau}^{-1}\left(\left[8 \tau, 1-2 \beta^{\frac{1}{8}}\right]\right)
$$

by using the Co-area formula

$$
\int_{\Pi_{16 \tau} \backslash \frac{B_{3 \beta^{\frac{1}{8}}(0)}}{(0)}}\left|v_{1,1}^{\tau}+v_{2,2}^{\tau}\right|\left|\nabla w^{\tau}\right| d z \leq \int_{8 \tau}^{1-2 \beta^{\frac{1}{8}}} \int_{w_{\tau}^{-1}(s)}\left|v_{1,1}^{\tau}+v_{2,2}^{\tau}\right| d H^{1} z d s \leq 4 \pi .
$$

Thus using (4.12)

$$
\int_{\Pi_{16 \tau} \backslash \frac{B_{3 \beta \frac{1}{8}}^{(0)}}{}}\left|v_{1,1}^{\tau}+v_{2,2}^{\tau}\right| d z \leq 8 \pi
$$


By Step 2 this implies $V\left(\nabla u, \Pi_{16 \tau} \backslash \overline{B_{3 \beta^{\frac{1}{8}}}(0)}\right) \leq 16 \pi$ and as $\tau$ is arbitrary $V\left(\nabla u, \Omega \backslash \overline{B_{3 \beta \frac{1}{8}}(0)}\right) \leq 16 \pi$.

Lemma 4.6. Let $\Omega$ be a convex domain and $\left|\Omega \triangle B_{1}(0)\right| \leq \beta$.

Let $u(x)=d(x, \partial \Omega)$ and $\eta(x)=1-8 \beta^{\frac{3}{32}}+|x|$. Define $\Gamma:=\{x: u(x)=\eta(x)\}$, we will show $\Gamma$ is the boundary of a convex set with $H^{1}(\Gamma) \leq c \beta^{\frac{3}{32}}$,

$$
\Gamma \subset N_{c \beta \frac{3}{16}}\left(\partial B_{4 \beta \frac{3}{32}}(0)\right)
$$

and for any $\varepsilon \in\left(0, \beta^{\frac{3}{16}}\right]$

$$
\left|N_{2 \varepsilon}(\Gamma)\right| \leq c \varepsilon \beta^{\frac{3}{32}}
$$

Proof of Lemma.

Step 1 . We will show $\Pi:=\{x \in \Omega: \eta(x) \leq u(x)\}$ is convex.

Proof of Step 1. Take $a, b \in \Pi$ and pick $\lambda \in[0,1]$. Since $u$ is concave $u(\lambda a+$ $(1-\lambda) b) \geq \lambda u(a)+(1-\lambda) u(b)$ and since $\eta$ is convex $\eta(\lambda a+(1-\lambda) b) \leq$ $\lambda \eta(a)+(1-\lambda) \eta(b)$. Hence as $a, b \in \Pi, u(\lambda a+(1-\lambda) b) \geq \eta(\lambda a+(1-\lambda) b)$. Thus $[a, b] \subset \Pi$ and thus the set $\Pi$ is convex.

Step 2. We will establish (4.42).

Proof of Step 2. Let $x \in \Gamma$ and let $b_{x} \in \partial \Omega$ be such that $\left|x-b_{x}\right|=u(x)$. So

$$
1-8 \beta^{\frac{3}{32}}+|x|=\left|b_{x}-x\right| .
$$

And thus $1-8 \beta^{\frac{3}{32}}+|x| \geq\left|b_{x}\right|-|x|$, so using (4.2)

$$
2|x| \geq\left|b_{x}\right|-1+8 \beta^{\frac{3}{32}} \geq 8 \beta^{\frac{3}{32}}-c \sqrt{\beta} .
$$

Also from (4.44) we have

$$
|x|=\left|b_{x}-x\right|-\left(1-8 \beta^{\frac{3}{32}}\right) \stackrel{(4.2)}{\leq} 8 \beta^{\frac{3}{32}}+\sqrt{\beta} .
$$

Now using Lemma 4.4, since $\nabla u(x)=\frac{x-b_{x}}{\left|x-b_{x}\right|}$ so

$$
\left|\frac{x}{|x|}-\frac{b_{x}}{\left|b_{x}\right|}\right| \underset{\substack{(4.45),(4.4) \\ \leq} \beta^{\frac{3}{32}}}{\leq}\left|\frac{b_{x}-x}{\left|b_{x}-x\right|}-\frac{b_{x}}{\left|b_{x}\right|}\right|+\left|\frac{x-b_{x}}{\left|x-b_{x}\right|}+\frac{x}{|x|}\right|
$$

so

$$
\left|1-\frac{b_{x}}{\left|b_{x}\right|} \cdot \frac{x}{|x|}\right|=2^{-1}\left|\frac{b_{x}}{\left|b_{x}\right|}-\frac{x}{|x|}\right|^{2} \leq c \beta^{\frac{3}{16}} .
$$


Again by Lemma 4.4 we have

$$
\begin{aligned}
|| b_{x}-x\left|+\left(x-b_{x}\right) \cdot \frac{x}{|x|}\right| & \leq\left|x-b_{x}\right|\left|\frac{x-b_{x}}{\left|x-b_{x}\right|}+\frac{x}{|x|}\right| \\
& \leq 2\left|\nabla u(x)+\frac{x}{|x|}\right| \\
& \stackrel{(4.4)}{\leq} c \beta^{\frac{3}{16}}
\end{aligned}
$$

and thus

$$
\begin{aligned}
\mid 2 x \cdot \frac{x}{|x|}-8 \beta^{\frac{3}{32} \mid} & \stackrel{(4.46)}{\leq}\left|-8 \beta^{\frac{3}{32}}+1-\frac{b_{x}}{\left|b_{x}\right|} \cdot \frac{x}{|x|}+2 x \cdot \frac{x}{|x|}\right|+c \beta^{\frac{3}{16}} \\
& =\left|1-8 \beta^{\frac{3}{32}}+\right| x\left|-\left(\frac{b_{x}}{\left|b_{x}\right|}-x\right) \cdot \frac{x}{|x|}\right|+c \beta^{\frac{3}{16}} \\
& \stackrel{(4.44)}{=}|| b_{x}-x\left|-\left(\frac{b_{x}}{\left|b_{x}\right|}-x\right) \cdot \frac{x}{|x|}\right|+c \beta^{\frac{3}{16}} \\
& \stackrel{(4.2)}{\leq}|| b_{x}-x\left|+\left(x-b_{x}\right) \cdot \frac{x}{|x|}\right|+c \beta^{\frac{3}{16}} \\
& \stackrel{(4.47)}{\leq} c \beta^{\frac{3}{16}}
\end{aligned}
$$

hence $|2| x\left|-8 \beta^{\frac{3}{32}}\right| \leq c \beta^{\frac{3}{16}}$ for any $x \in \Gamma$, so (4.42) is established.

Since (4.42) implies the diameter of $\Pi$ is bounded by $c \beta^{\frac{3}{32}}$ and since $\Pi$ is a convex set it follows immediately that $H^{1}(\Gamma) \leq c \beta^{\frac{3}{32}}$.

Now the set $\Gamma$ equipped with the Euclidean norm is a bounded compact metric space. So by applying the 5r Covering Theorem [20, Theorem 2.1] we can find a disjoint collection of balls $B_{2 \varepsilon}\left(x_{1}\right), B_{2 \varepsilon}\left(x_{2}\right), \ldots B_{2 \varepsilon}\left(x_{M}\right)$ with $x_{1}, x_{2}, \ldots x_{M} \in$ $\Gamma$ such that $\Gamma \subset \bigcup_{i=1}^{n} B_{10 \varepsilon}\left(x_{i}\right)$. This implies $N_{2 \varepsilon}(\Gamma) \subset \bigcup_{i=1}^{n} B_{20 \varepsilon}\left(x_{i}\right)$. Since $H^{1}(\Gamma) \leq c \beta^{\frac{3}{32}}$ so $M \leq c \varepsilon^{-1} \beta^{\frac{3}{32}}$ and thus $\left|N_{2 \varepsilon}(\Gamma)\right| \leq c \varepsilon \beta^{\frac{3}{32}}$ which establishes (4.43).

Lemma 4.7. Let $\Omega$ be a convex set. Let $\beta=\left|\Omega \triangle B_{1}(0)\right|$. Let

$$
w(z):=\min \left\{d(z, \partial \Omega), 1-8 \beta^{\frac{3}{32}}+|z|\right\} .
$$

We will show $\nabla w \in S B V\left(\Omega: S^{1}\right)$ and

$$
\int_{J_{\nabla w} \cap \Omega}\left|\nabla w^{+}-\nabla w^{-}\right|^{3} d H^{1} \leq c \beta^{\frac{3}{32}} .
$$


Proof. By Lemma 4.5 we know $\nabla u \in B V\left(\Omega \backslash B_{3 \beta^{\frac{1}{3}}}(0)\right)$ and $V\left(\nabla u, \Omega \backslash B_{3 \beta^{\frac{1}{3}}}(0)\right) \leq$ $8 \pi$. This implies

$$
\int_{\left(\Omega \backslash B_{3 \beta^{\frac{1}{8}}}(0)\right) \cap S_{\nabla u}}\left|\nabla u^{+}-\nabla u^{-}\right| d H^{1} \leq 8 \pi .
$$

Now by Lemma 4.4 (4.4) for any $x \in\left(\Omega \backslash B_{3 \beta^{\frac{1}{8}}}(0)\right) \cap S_{\nabla u}$ we have $\left|\nabla u^{+}(x)-\nabla u^{-}(x)\right| \leq$ $c \beta^{\frac{3}{16}}$. So

$$
\begin{gathered}
\left.\left.\int_{\left(\Omega \backslash B_{3 \beta^{\frac{1}{8}}}(0)\right) \cap S_{\nabla u}}\left|\nabla u^{+}-\nabla u^{-}\right|^{3} d H^{1} \leq c \beta^{\frac{3}{8}} \int_{(\Omega \backslash B} \underset{3 \beta^{\frac{1}{8}}}{ } \mid \nabla\right)\right) \cap S_{\nabla u}\left|\nabla u^{+}-\nabla u^{-}\right| d H^{1} \\
\stackrel{\text { (4.49) }}{\leq} c \beta^{\frac{3}{8}} .
\end{gathered}
$$

As in Lemma 4.6 let $\Pi:=\left\{x: u(x) \leq 1-8 \beta^{\frac{3}{32}}+|x|\right\}$ and $\Gamma:=\partial \Pi$. Since $\Pi$ is convex it is also a set of finite perimeter. Let $\eta(z)=1-8 \beta^{\frac{3}{32}}+|x|$, it is clear $w(z)=$

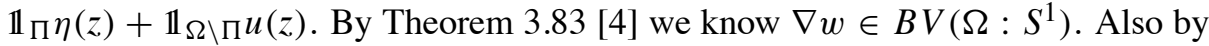
Lemma 4.6, $H^{1}(\Gamma) \leq c \beta^{\frac{3}{32}}$. Now for any $x \in \Gamma$, since $\nabla w^{+}(x), \nabla w^{-}(x) \in S^{1}$, $\left|\nabla w^{+}(x)-\nabla w^{-}(x)\right| \leq 2$. So

$$
\begin{aligned}
& \int_{J_{\nabla w}}\left|\nabla w^{+}-\nabla w^{-}\right|^{3} d H^{1}=\int_{J_{\nabla w} \cap(\Omega \backslash \Pi)}\left|\nabla w^{+}-\nabla w^{-}\right|^{3} d H^{1} \\
&+\int_{J_{\nabla w} \cap \Pi}\left|\nabla w^{+}-\nabla w^{-}\right|^{3} d H^{1} \\
& \stackrel{(4.50)}{\leq} c \beta^{\frac{3}{8}}+8 H^{1}(\Gamma) \\
& \leq c \beta^{\frac{3}{32}}
\end{aligned}
$$

\subsection{Proof of Proposition 4.1 completed}

By Lemma 4.7 we know that $w \in B V\left(\Omega, S^{1}\right)$ we can apply Theorem 1 of [8] or Corollary 1.1 [23] to find a sequence $u^{\epsilon}$ that satisfies $u^{\epsilon}(z)=0$ and $\nabla u^{\epsilon}(z) \cdot \eta_{z}=1$ for $z \in \partial \Omega$ (where $\eta_{z}$ is the inward pointing unit normal to $\partial \Omega$ at $z$ ) such that

$$
\left.\left.\underset{\epsilon \rightarrow 0}{\limsup } \int_{\Omega} \epsilon^{-1}|1-| \nabla u^{\epsilon}\right|^{2}\right|^{2}+\epsilon\left|\nabla^{2} u^{\epsilon}\right|^{2} d z \leq \int_{J_{\nabla w} \cap \Omega}\left|\nabla w^{+}-\nabla w^{-}\right|^{3} d H^{1}
$$




\subsection{Proof of Corollary 1.3}

Let $\beta=\inf _{a \in \Omega}\left|\Omega \triangle B_{1}(a)\right|$. Without loss of generality we can assume $\left|\Omega \triangle B_{1}(0)\right| \leq$ $2 \beta$. So by Proposition 4.1 we can find $\epsilon_{0} \in(0,1)$ such that for $\epsilon \in\left(0, \epsilon_{0}\right)$, any minimiser $u^{\epsilon}$ of $I_{\epsilon}$ defined on $\Omega$ satisfies

$$
\left.\left.\int_{\Omega} \epsilon^{-1}|1-| \nabla u^{\epsilon}\right|^{2}\right|^{2}+\epsilon\left|\nabla^{2} u^{\epsilon}\right|^{2} d z \leq c \beta^{\frac{3}{32}} .
$$

So we can apply Theorem 1.1 to conclude that

$$
\int_{\Omega}\left|\nabla u^{\epsilon}(z)+\frac{z}{|z|}\right|^{2} d z \leq c \beta^{\frac{1}{5462}} .
$$

Applying Lemma 4.4 we have

$$
\int_{\Omega \backslash B \underset{\beta^{\frac{1}{8}}}{ }(0)}\left|\nabla u^{\epsilon}-\nabla \zeta\right|^{2} \leq c \beta^{\frac{1}{5462}} .
$$

Now

$$
\begin{aligned}
\int_{B_{\beta^{\frac{1}{8}}}(0)}\left|\nabla u^{\epsilon}-\nabla \zeta\right|^{2} d z & \leq \int_{B_{\beta^{\frac{1}{8}}}(0)}\left|\nabla u^{\epsilon}\right|^{2}+2\left|\nabla u^{\epsilon}\right|+1 d z \\
& \leq \int_{B_{\beta^{\frac{1}{8}}}(0)}\left(\left.|1-| \nabla u^{\epsilon}\right|^{2} \mid+c\right) d z \\
& \stackrel{(4.51)}{\leq} c \beta^{\frac{3}{32}}
\end{aligned}
$$

together with (4.52) this gives $\left\|u^{\epsilon}-\zeta\right\|_{W^{1,2}(\Omega)} \leq c \beta \frac{1}{5462}$.

\section{Proof of Corollary 1.2}

In this section we will show that given a convex domain $\Omega$ with $C^{2}$ boundary with curvature bounded above by $\epsilon^{-\frac{1}{2}}$ and that satisfies $\left|B_{1}(0) \triangle \Omega\right| \leq \beta$ we will construct a function $u$ with $I_{\epsilon}(u) \leq \beta^{\frac{3}{16}}$. This is the content of Proposition 5.1 below. The proof of Corollary 1.2 will follow easily from this.

Proposition 5.1. Let $\Omega$ be a convex body with $C^{2}$ boundary and with curvature bounded above by $\epsilon^{-\frac{1}{2}}$ and $\left|\Omega \triangle B_{1}(0)\right| \leq \beta$. Let $\epsilon \in\left(0, \frac{\beta^{\frac{1}{2}}}{4}\right]$. There exists $a$ function $C^{2}$ function $\xi: \Omega \rightarrow \mathbb{R}$ which satisfies $\nabla \xi(z) \cdot \eta_{z}=1$ (where $\eta_{z}$ is the inward pointing unit normal to $\partial \Omega$ at $z$ ), $\xi(z)=0$ for $z \in \partial \Omega$ and for which

$$
\left.\left.\int_{\Omega} \epsilon^{-1}|1-| \nabla \xi\right|^{2}\right|^{2}+\epsilon\left|\nabla^{2} \xi\right|^{2} d z \leq c \beta^{\frac{3}{32}} .
$$




\subsection{Proof of Proposition 5.1}

We begin with a preliminary lemma.

Lemma 5.2. Let $\phi: \mathbb{R}_{+} \rightarrow \mathbb{R}_{+}$be a continuous function. Let $\rho$ denote the standard convolution kernel, i.e. $\int \rho d x=1$ and $\operatorname{Spt} \rho \subset B_{\frac{3}{2}}(0)$ and define $\rho_{h}(z):=$ $h^{-2} \rho\left(h^{-1} z\right)$.

Suppose $f: \mathbb{R}^{n} \rightarrow \mathbb{R}$ be an affine function. If $g(x)=f * \rho_{\phi(x)}(x)$ then

$$
g(x)=f(x) \text { for all } x \in \mathbb{R}^{n} .
$$

Proof of Lemma. Let $\eta=\nabla f$. As $f$ is affine $f(x-y)=f(x)-\eta \cdot y$

$$
\begin{aligned}
g(x) & =\int f(x-y)(\phi(x))^{-2} \rho\left(\phi(x)^{-1} y\right) d y \\
& =\int(f(x)-\eta \cdot y)(\phi(x))^{-2} \rho\left(\phi(x)^{-1} y\right) d y \\
& =f(x) .
\end{aligned}
$$

Lemma 5.3. Let $\epsilon>0$. Suppose $\Omega$ is a convex body with $C^{2}$ boundary and with curvature bounded above by $\epsilon^{-\frac{1}{2}}$. Let $u(x)=d(x, \partial \Omega)$. Let $\rho$ be the standard convolution kernel and $\rho_{\epsilon}(z):=\rho\left(\frac{z}{\epsilon}\right) \epsilon^{-2}$. We will construct a function $\psi: \Omega \cap$ $N_{8 \epsilon}(\partial \Omega) \rightarrow \mathbb{R}$ with $\psi=0$ on $\partial \Omega$ which satisfies the following properties

$$
\begin{gathered}
\left.\left.\int_{\Omega \cap N_{8 \epsilon}(\partial \Omega)}|1-| \nabla \psi\right|^{2}\right|^{2} d z \leq c \epsilon^{2}, \\
\int_{\Omega \cap N_{8 \epsilon}(\partial \Omega)}\left|\nabla^{2} \psi\right|^{2} d z \leq c, \\
\psi(z)=\left[u * \rho_{\epsilon}\right](z) \text { for any } z \in \Omega \backslash N_{8 \epsilon}(\partial \Omega)
\end{gathered}
$$

and

$$
\nabla \psi(z)=\eta_{z} \text { for each } z \in \partial \Omega
$$

Proof. Let $w: \mathbb{R}_{+} \rightarrow \mathbb{R}_{+}$be a smooth monotone function with the following properties

$$
w(z)= \begin{cases}z & \text { for } z \in\left[0, \frac{\epsilon}{3}\right) \\ \epsilon & \text { for } z \geq \epsilon\end{cases}
$$

and $\sup |\ddot{w}| \leq c \epsilon^{-1}$.

For any $x \in \Omega \cap N_{8 \epsilon}(\partial \Omega)$ define

$$
\phi(x)=w(u(x)) .
$$


We will convolve the function $u$ with convolution kernel $\rho_{\phi(x)}(z):=\rho\left(\frac{z}{\phi(x)}\right) /(\phi(x))^{2}$. Since the convolution kernel varies with $x$, when we differentiate $u * \rho_{\phi(x)}$, the derivative will involve a term with the derivative of $\rho_{\phi(x)}$. For this reason we need to calculate various partial derivatives of $\rho_{\phi}(x)$.

Since the curvature of $\partial \Omega$ is bounded above by $\epsilon^{-\frac{1}{2}}$. For any $x \in \Omega \cap N_{8 \epsilon}(\partial \Omega)$ we have that there is one unique $b_{x} \in \partial \Omega$ such that $\left|x-b_{x}\right|=u(x)$. We define $\varsigma_{x}=\frac{x-b_{x}}{\left|x-b_{x}\right|}$. Let $R=\left(\begin{array}{cc}0 & -1 \\ 1 & 0\end{array}\right)$ and define $\omega_{x}=R \varsigma_{x}$.

Note $\varsigma_{x}=\eta_{b_{x}}$, i.e. the inward pointing unit normal to $\partial \Omega$ at $b_{x}$. Note also that for all small enough $h, b_{x}=b_{x+h \varsigma_{x}}$ so $u\left(x+h \varsigma_{x}\right)=h+u(x)$. Thus

$$
\begin{aligned}
\phi_{, \zeta_{x}}(x) & =\lim _{h \rightarrow 0} \frac{\phi\left(x+h \varsigma_{x}\right)-\phi(x)}{h} \\
& =\lim _{h \rightarrow 0} \frac{w(u(x)+h)-w(u(x))}{h} \\
& =\dot{w}(u(x)) .
\end{aligned}
$$

Note also that since $|\nabla u(x)|=1$ and $u_{, \varsigma_{x}}(x)=\lim _{h \rightarrow 0} \frac{u\left(x+h \varsigma_{x}\right)-u(x)}{h}=1$ so

$$
u_{, \omega_{x}}(x)=\lim _{h \rightarrow 0} \frac{u\left(x+h \omega_{x}\right)-u(x)}{h}=0 .
$$

Thus

$$
\phi_{, \omega_{x}}(x)=\dot{w}(u(x)) u_{, \omega_{x}}(x)=0 .
$$

Hence

$$
\begin{aligned}
\frac{\partial}{\partial \varsigma_{x}}\left(\rho_{\phi(x)}(z)\right) & =\frac{\partial}{\partial \varsigma_{x}}\left(\rho\left(\frac{z}{\phi(x)}\right)(\phi(x))^{-2}\right) \\
& =-\nabla \rho\left(\frac{z}{\phi(x)}\right) \cdot z \frac{\phi_{, \varsigma_{x}}(x)}{(\phi(x))^{4}}-2 \rho\left(\frac{z}{\phi(x)}\right) \frac{\phi_{, \varsigma_{x}}(x)}{(\phi(x))^{3}}
\end{aligned}
$$

and

$$
\frac{\partial}{\partial \omega_{x}}\left(\rho_{\phi(x)}(z)\right)=0
$$

Define

$$
\psi(x):=\left\{\begin{array}{ll}
\int u(x-z) \rho_{\phi(x)}(z) d z & \text { for } x \in \Omega \\
0 & \text { for } x \notin \Omega
\end{array} .\right.
$$

Now

$$
\begin{aligned}
\psi_{, \zeta_{x}}(x) \stackrel{(5.9)}{=} & \int u_{, \varsigma_{x}}(x-z) \rho_{\phi(x)}(z) d z \\
& -\int u(x-z)\left(\nabla \rho\left(\frac{z}{\phi(x)}\right) \cdot z \frac{\phi_{, \varsigma_{x}}(x)}{(\phi(x))^{4}}+2 \rho\left(\frac{z}{\phi(x)}\right) \frac{\phi_{, \zeta_{x}}(x)}{(\phi(x))^{3}}\right) d z .
\end{aligned}
$$


In the same way it is easy to see $\psi_{, \omega_{x}}(x)=\int u_{, \omega_{x}}(x-z) \rho_{\phi(x)}(z) d z$ and so

$$
\psi_{, \zeta_{x} \omega_{x}}(x)=\int u_{, \omega_{x} \zeta_{x}}(x-z) \rho_{\phi(x)}(z) d z+\int u_{, \omega_{x}}(x-z) \frac{\partial}{\partial \zeta_{x}}\left(\rho_{\phi(x)}(z)\right) d z .
$$

And

$$
\psi_{, \omega_{x} \omega_{x}}(x)=\int u_{, \omega_{x} \omega_{x}}(x-z) \rho_{\phi(x)}(z) d z
$$

Finally

$$
\begin{aligned}
& \psi_{, \varsigma_{x} \zeta_{x}}(x)=\int u_{, \zeta_{x} \varsigma_{x}}(x-z) \rho_{\phi(x)}(z)+2 u_{, \zeta_{x}}(x-z) \frac{\partial}{\partial \zeta_{x}}\left(\rho_{\phi(x)}(z)\right) d z \\
& +\int u(x-y) \frac{\partial^{2}}{\partial^{2} \varsigma_{x}}\left(\rho_{\phi(x)}(z)\right) d z
\end{aligned}
$$

each term will be estimated later in Step 4.

Step 1 . We will show

$$
\left|\nabla^{2} u(x)\right| \leq c \epsilon^{-\frac{1}{2}} \text { for any } x \in N_{\frac{\sqrt{\epsilon}}{3}}(\partial \Omega) .
$$

Proof of Step 1. Let $b_{x} \in \partial \Omega$ be such that $\operatorname{dist}(x, \partial \Omega)=\left|x-b_{x}\right|$. We start by showing

$$
|\nabla u(x)-\nabla u(y)| \leq c \epsilon^{-\frac{1}{2}}|x-y| \text { for any } x \in N_{\frac{\sqrt{\epsilon}}{3}}(\partial \Omega), y \in B_{\frac{\epsilon}{6}}(x) .
$$

Now recall $\frac{y-b_{y}}{\left|y-b_{y}\right|}=\eta_{b_{y}}, \frac{x-b_{x}}{\left|x-b_{x}\right|}=\eta_{b_{x}}$. We have two cases to consider. Firstly the case that $\left(b_{x}+\mathbb{R}_{+} \eta_{b_{x}}\right) \cap\left(b_{y}+\mathbb{R}_{+} \eta_{b_{y}}\right)=\emptyset$. In this case since $\Omega$ is convex this implies $\eta_{b_{x}}=\eta_{b_{y}}$. Thus as $|\nabla u(x)-\nabla u(y)|=\left|\frac{y-b_{y}}{\left|y-b_{y}\right|}-\frac{x-b_{x}}{\left|x-b_{x}\right|}\right|=\left|\eta_{b_{x}}-\eta_{b_{y}}\right|=$ 0 so (5.16) is established.

Now suppose we have the case that $\pi:=\left(b_{x}+\mathbb{R}_{+} \eta_{b_{x}}\right) \cap\left(b_{y}+\mathbb{R}_{+} \eta_{b_{y}}\right) \neq \emptyset$. Then let

$$
\theta=\arccos \left(\frac{b_{y}-y}{\left|b_{y}-y\right|} \cdot \frac{b_{x}-x}{\left|b_{x}-x\right|}\right) .
$$

Since the curvature of $\partial \Omega$ is bounded by $\epsilon^{-\frac{1}{2}}$ we know that $\pi \notin N_{\sqrt{\epsilon}}(\partial \Omega)$. Consider the triangle whose corners are $x, y, \pi$, which we denote by $T(x, y, \pi)$. The angle at corner $\pi$ is $\theta$. Note that $|x-y| \leq \frac{\epsilon}{6},|x-\pi| \geq \frac{\sqrt{\epsilon}}{2}$ and $|y-\pi| \geq \frac{\sqrt{\epsilon}}{2}$. So as ||$x-\pi|-| y-\pi|| \leq|x-y| \leq \frac{\epsilon}{6}$ we therefore know

$$
\frac{\epsilon^{2}}{36} \geq|| x-\pi|-| y-\pi||^{2}=|2| x-\pi|| y-\pi|-| x-\left.\pi\right|^{2}-|y-\pi|^{2} \mid .
$$


Thus by the law of cosines

$$
\begin{aligned}
2|x-\pi||y-\pi| \cos \theta & =|x-\pi|^{2}+|y-\pi|^{2}-|x-y|^{2} \\
& \geq|x-\pi|^{2}+|y-\pi|^{2}-\frac{\epsilon^{2}}{36} \\
& \geq 2|x-\pi||y-\pi|-\frac{\epsilon^{2}}{36} .
\end{aligned}
$$

Which implies $\cos \theta \geq 1-c \epsilon$ and so $|\theta| \leq c \sqrt{\epsilon}$.

Let $\tilde{y}:=\left[b_{y}, \pi\right] \cap \partial B_{|x-\pi|}(x)$. Since $|\theta| \leq c \sqrt{\epsilon}$ we have $|x-\tilde{y}| \leq \frac{11}{10}|x-y|$. Consider the triangle $T(x, \tilde{y}, \pi)$. Note the angle of this triangle at $\pi$ is $\theta$ and denoting the angle at $x$ by $\psi$ we have $\psi \sim \frac{\pi}{2}$.

Then by the law of sins

$$
\frac{|x-\tilde{y}|}{\sin \theta}=\frac{|\tilde{y}-\pi|}{\sin \psi} \geq \frac{|\tilde{y}-\pi|}{2} \geq \frac{\sqrt{\epsilon}}{4} .
$$

So $4 \frac{|x-\tilde{y}|}{\sqrt{\epsilon}} \geq \sin \theta$ which gives $|\theta| \leq \frac{c|x-\tilde{y}|}{\sqrt{\epsilon}} \leq \frac{c|x-y|}{\sqrt{\epsilon}}$. So as $\nabla u(x)=\frac{x-b_{x}}{\left|x-b_{x}\right|}$ and $\nabla u(y)=\frac{y-b_{y}}{\left|y-b_{y}\right|}$, hence (recalling the definition of $\theta$ from (5.17)) $|\nabla u(x)-\nabla u(y)| \leq$ $c \arccos (\nabla u(x) \cdot \nabla u(y)) \leq \frac{c|x-y|}{\sqrt{\epsilon}}$. So (5.16) is established. Thus letting $y \rightarrow x$ we have that $\left|\nabla^{2} u(x)\right| \leq c \epsilon^{-\frac{1}{2}}$ and this completes the proof of Step 1 .

Step 2. For any $x \in N_{16 \epsilon}(\partial \Omega) \cap \Omega$ we have

$$
\sup \left\{\left|\nabla u(z)-\varsigma_{x}\right|: z \in B_{16 u(x)}(x) \cap \Omega\right\} \leq c \epsilon^{-\frac{1}{2}} u(x) .
$$

Proof of Step 2. Since $\partial \Omega$ has curvature less than $\epsilon^{-\frac{1}{2}}$ for any $x_{1}, x_{2} \in \partial \Omega$, $\left[x_{1}, x_{1}+\epsilon^{\frac{1}{2}} \eta_{x_{1}}\right] \cap\left[x_{2}, x_{2}+\epsilon^{\frac{1}{2}} \eta_{x_{2}}\right]=\emptyset$. So for any $x_{1}, x_{2} \in B_{32 u(x)}(x) \cap \partial \Omega$, $\left|\eta_{x_{1}}-\eta_{x_{2}}\right| \leq \epsilon^{-\frac{1}{2}} H^{1}\left(B_{32 u(x)}(x) \cap \partial \Omega\right)$. Note as $\Omega \cap B_{32 u(x)}(x)$ is convex and $\partial \Omega \cap B_{32 u(x)}(x) \subset \partial\left(\Omega \cap B_{32 u(x)}(x)\right)$ so $H^{1}\left(\partial \Omega \cap B_{32 u(x)}(x)\right) \leq c u(x)$. Hence $\left|\eta_{x_{1}}-\eta_{x_{2}}\right| \leq c \epsilon^{-\frac{1}{2}} u(x) \leq c \sqrt{\epsilon}$ so it is clear that

$$
B_{16 u(x)}(x) \cap \Omega \subset \bigcup_{z \in \partial \Omega \cap B_{32 u(x)}(x)}\left[z, z+\sqrt{\epsilon} \eta_{z}\right] .
$$

For any $z \in B_{16 u(x)}(x) \cap \Omega$ we have $\nabla u(z)=\frac{z-b_{z}}{\left|z-b_{z}\right|}=\eta_{b_{z}}$ where $b_{z}$ is such that $\left|z-b_{z}\right|=d(z, \partial \Omega)$. So for any $z_{1}, z_{2} \in B_{16 u(x)}(x) \cap \Omega$ by (5.19) we have that $b_{z_{1}}, b_{z_{2}} \in \partial \Omega \cap B_{32 u(x)}(x)$, so $\left|\nabla u\left(z_{1}\right)-\nabla u\left(z_{2}\right)\right|=\left|\eta_{b_{z_{1}}}-\eta_{b_{z_{2}}}\right| \leq c \epsilon^{-\frac{1}{2}} u(x)$.

Step 3. For any $x \in N_{8 \epsilon}(\partial \Omega) \cap \Omega$ we have

$$
|| \nabla \psi(x)|-1| \leq c \sqrt{\epsilon} .
$$


And

$$
\lim _{y \rightarrow z} \nabla \psi(y)=\eta_{z}
$$

Proof of Step 3. From (5.11) we have

$$
\begin{aligned}
& \left|\psi_{, \zeta_{x}}(x)-1\right| \\
& \leq \overbrace{\left|\int\left(u_{,{ }_{\zeta}}(x-z)-1\right) \rho\left(\frac{z}{\phi(x)}\right)(\phi(x))^{-2} d z\right|}^{B} \\
& +\overbrace{\left|\int \frac{-u(x-z) \phi_{,{ }_{5}}(x)}{(\phi(x))^{3}}\left(\nabla \rho\left(\frac{z}{\phi(x)}\right) \cdot \frac{z}{\phi(x)}+2 \rho\left(\frac{z}{\phi(x)}\right)\right) d z\right|}^{C} .
\end{aligned}
$$

Now for any $z \in \operatorname{Spt} \rho_{\phi(x)}$ we have that $\nabla u(x-z)=u_{, \varsigma_{x}}(x-z) \varsigma_{x}+u_{, \omega_{x}}(x-z) \omega_{x}$. As $\operatorname{Spt} \rho_{\phi(x)} \subset B_{2 \phi(x)}(0) \subset B_{2 u(x)}(0)$ so for any $z \in \operatorname{Spt} \rho_{\phi(x)}$ by (5.18) from Step 2 we have $\left|\nabla u(x-z)-\varsigma_{x}\right| \leq c \epsilon^{-\frac{1}{2}} u(x)$ and thus

$$
\left|u,,_{x}(x-z)-1\right| \leq c \epsilon^{-\frac{1}{2}} u(x) \text { for any } z \in \operatorname{Spt} \rho_{\phi(x)} .
$$

So (noting $u(x) \leq c \phi(x)$ for any $x \in N_{8 \epsilon}(\partial \Omega) \cap \Omega$ )

$$
B \leq c u(x) \epsilon^{-\frac{1}{2}}<c \phi(x) \epsilon^{-\frac{1}{2}} .
$$

Also defining $w=f_{B_{\phi(x)}(x)} \nabla u d z$

$$
\left|w-\varsigma_{x}\right|=\left|f_{B_{\phi(x)}(x)}\left(\nabla u(z)-\varsigma_{x}\right) d z\right| \stackrel{(5.18)}{\leq} c \epsilon^{-\frac{1}{2}} \phi(x) .
$$

So by Poincaré's inequality there exists affine function $l_{w}$ with $\nabla l_{w}=w$

$$
\begin{aligned}
f_{B_{\phi(x)}(x)}\left|u(z)-l_{w}(z)\right| d z & \leq c \phi(x) f_{B_{\phi(x)}(x)}|\nabla u(z)-w| d z \\
& \leq c \phi(x)\left(f_{B_{\phi(x)}(x)}\left|\nabla u(z)-\varsigma_{x}\right| d z+c\left|w-\varsigma_{x}\right|\right) \\
& \leq \epsilon^{-\frac{1}{2}(\phi(x),(5.24))^{2} .}
\end{aligned}
$$

Now using (5.24), again for the appropriate choice of affine function $l_{\zeta_{x}}$ with $\nabla l_{\zeta_{x}}=$ $\varsigma_{x}$, we have by Poincaré's inequality

$$
f_{B_{\phi(x)}(x)}\left|l_{\varsigma x}(z)-l_{w}(z)\right| d z \leq c \phi(x) f_{B_{\phi(x)}}\left|w-\varsigma_{x}\right| d z \stackrel{(5.24)}{\leq} c \epsilon^{-\frac{1}{2}}(\phi(x))^{2}
$$


with (5.25) this gives

$$
f_{B_{\phi(x)}(x)}\left|l_{\zeta x}(z)-u(z)\right| d z \leq c \epsilon^{-\frac{1}{2}}(\phi(x))^{2} .
$$

Let $g$ be defined by $g(y)=l_{\zeta_{x}} * \rho_{\phi(y)}(y)$. Note by Lemma 5.2 we have $\nabla g(y)=\varsigma_{x}$ for any $y \in \Omega$ and hence $g_{, \varsigma_{x}}(x)=1$. As

$$
\begin{aligned}
g_{\zeta_{x}}(x)= & \int \rho\left(\frac{z}{\phi(x)}\right)(\phi(x))^{-2} d z \\
& -\int \frac{l_{\zeta_{x}}(x-z)}{(\phi(x))^{3}} \phi_{\zeta_{x}}(x)\left(\nabla \rho\left(\frac{z}{\phi(x)}\right) \cdot z(\phi(x))^{-1}+2 \rho\left(\frac{z}{\phi(x)}\right)\right) d z \\
= & 1-\int \frac{l_{\zeta_{x}}(x-z)}{(\phi(x))^{3}} \phi_{, \zeta_{x}}(x)\left(\nabla \rho\left(\frac{z}{\phi(x)}\right) \cdot z(\phi(x))^{-1}+2 \rho\left(\frac{z}{\phi(x)}\right)\right) d z .
\end{aligned}
$$

Thus

$$
0=\int \frac{l_{\zeta_{x}}(x-z)}{(\phi(x))^{3}} \phi_{, \zeta_{x}}(x)\left(\nabla \rho\left(\frac{z}{\phi(x)}\right) \cdot z(\phi(x))^{-1}+2 \rho\left(\frac{z}{\phi(x)}\right)\right) d z .
$$

So

$$
\begin{aligned}
C & \leq \int \frac{\left|l_{S_{x}}(x-z)-u(x)\right|}{(\phi(x))^{3}}\left|\phi_{S_{x}}(x)\left(\nabla \rho\left(\frac{z}{\phi(x)}\right) \cdot z(\phi(x))^{-1}+2 \rho\left(\frac{z}{\phi(x)}\right)\right)\right| d z \\
& \leq c(\phi(x))^{-3} \int_{B_{\phi(x)}(x)}\left|l_{\zeta_{x}}(z)-u(z)\right| d z \\
& \stackrel{(5.26)}{\leq} c \epsilon^{-\frac{1}{2}} \phi(x) .
\end{aligned}
$$

Since $x \in N_{8 \epsilon}(\partial \Omega) \cap \Omega$ we know $\phi(x) \leq c \epsilon$ applying (5.27) and (5.23) to (5.22) gives

$$
\left|\psi_{, \zeta_{x}}(x)-1\right| \leq c \epsilon^{-\frac{1}{2}} \phi(x) \leq c \sqrt{\epsilon} .
$$

Now using that $u_{, \omega_{x}}(x)=0$ we have that

$$
\begin{aligned}
\left|\psi_{, \omega_{x}}(x)\right| & \leq\left|\int u_{, \omega_{x}}(x-z) \rho_{\phi(x)}(z) d z\right| \\
& \leq \int\left|u_{, \omega_{x}}(x-z)-u_{, \omega_{x}}(x)\right| \rho_{\phi(x)}(z) d z \\
& \leq c \epsilon^{-\frac{1}{2}} \phi(x) \int \rho_{\phi(x)}(z) d z \\
& \leq c \epsilon^{\frac{1}{2}} \phi(x) .
\end{aligned}
$$


Thus $\left|\nabla \psi(x)-\varsigma_{x}\right| \leq c \sqrt{\epsilon}$ and (5.20) follows easily. Also for (5.28), (5.29) we know $\left|\nabla \psi(x)-\eta_{b_{x}}\right| \leq c \epsilon^{-\frac{1}{2}} \phi(x)$ and (5.21) follows. This completes the proof of Step 3.

Step 4. We will show

$$
\left|\nabla^{2} \psi(x)\right| \leq c \epsilon^{-\frac{1}{2}} \text { for any } x \in N_{8 \epsilon}(\partial \Omega) \cap \Omega .
$$

Proof Step 4. We will estimate the terms in (5.14) one by one. First note

$$
\begin{aligned}
& \int u(x-y) \frac{\partial^{2}}{\partial^{2} \varsigma_{x}}\left(\rho_{\phi(x)}(z)\right) d z \\
& =\int u(x-z) \partial_{\varsigma_{x}}\left(\sum_{k=1}^{2}-\rho_{, k}\left(\frac{z}{\phi(x)}\right) \frac{z_{k} \phi_{, \varsigma_{x}}(x)}{(\phi(x))^{4}}-2 \rho\left(\frac{z}{\phi(x)}\right) \frac{\phi_{, \varsigma_{x}}(x)}{(\phi(x))^{3}}\right) d z \\
& =\int u(x-z)\left(\sum_{k, l=1}^{2} \rho_{, k l}\left(\frac{z}{\phi(x)}\right) \frac{\left(\phi, \zeta_{x}(x)\right)^{2}}{(\phi(x))^{6}} z_{k} z_{l}\right. \\
& -\sum_{k=1}^{2} \rho_{, k}\left(\frac{z}{\phi(x)}\right) z_{k} \partial_{\zeta_{x}}\left(\frac{\phi, \varsigma_{x}(x)}{(\phi(x))^{4}}\right) \\
& +2 \sum_{m=1}^{2} \rho_{, m}\left(\frac{z}{\phi(x)}\right) z_{m} \frac{\left(\phi, \varsigma_{x}(x)\right)^{2}}{(\phi(x))^{5}} \\
& \left.-2 \rho\left(\frac{z}{\phi(x)}\right) \partial_{\varsigma_{x}}\left(\frac{\phi, \varsigma_{x}(x)}{(\phi(x))^{3}}\right)\right) d z
\end{aligned}
$$

Note

$$
\partial_{\varsigma_{x}}\left(\frac{\phi_{, \zeta_{x}}(x)}{(\phi(x))^{3}}\right)=\frac{-3\left(\phi_{, \varsigma_{x}}(x)\right)^{2}}{(\phi(x))^{4}}+\frac{\phi_{, \zeta_{x} \zeta_{x}}(x)}{(\phi(x))^{3}}
$$

and

$$
\partial_{\zeta_{x}}\left(\frac{\phi_{, \zeta_{x}}(x)}{(\phi(x))^{4}}\right)=\frac{-4\left(\phi_{, \zeta_{x}}(x)\right)^{2}}{(\phi(x))^{5}}+\frac{\phi_{, \zeta_{x} \zeta_{x}}(x)}{(\phi(x))^{4}} .
$$

So

$$
\begin{aligned}
\int u(x-y) \frac{\partial^{2}}{\partial^{2} \varsigma_{x}}\left(\rho_{\phi(x)}(z)\right) d z \\
=\int u(x-z)\left(\left(\nabla^{2} \rho\left(\frac{z}{\phi(x)}\right): z \otimes z\right) \frac{\left(\phi, \varsigma_{x}(x)\right)^{2}}{(\phi(x))^{6}}\right. \\
+\left(-\frac{\phi_{, \varsigma_{x} \varsigma_{x}}(x)}{(\phi(x))^{4}}+\frac{6\left(\phi_{, \varsigma_{x}}(x)\right)^{2}}{(\phi(x))^{5}}\right) \nabla \rho\left(\frac{z}{\phi(x)}\right) \cdot z \\
\left.+\left(\frac{6\left(\phi, \varsigma_{x}(x)\right)^{2}}{(\phi(x))^{4}}-\frac{2 \phi, \varsigma_{x} \varsigma_{x}(x)}{(\phi(x))^{3}}\right) \rho\left(\frac{z}{\phi(x)}\right)\right) d z
\end{aligned}
$$


From Step 2 (5.26) we know the existence of an affine function $l_{\zeta x}$ with $\nabla l_{\zeta x}=\varsigma_{x}$ with $f_{B_{\phi(x)}(x)}\left|u-l_{\zeta_{x}}\right| d z \leq c \epsilon^{\frac{1}{2}} \phi(x)$. Let $g(x):=l_{\zeta_{x}} * \rho_{\phi(x)}(x)$; by Lemma 5.2 we know $g_{,{ }_{5} \varsigma_{x}}(x)=0$. By following through the same calculation that gave (5.31), we have

$$
\begin{aligned}
0=\int l_{\zeta_{x}}(x-z)( & \left(\nabla^{2} \rho\left(\frac{z}{\phi(x)}\right): z \otimes z\right) \frac{\left(\phi_{, \zeta_{x}}(x)\right)^{2}}{(\phi(x))^{6}} \\
+ & \left(-\frac{\phi_{, \varsigma_{x} \zeta_{x}(x)}}{(\phi(x))^{4}}+\frac{6\left(\phi_{, \zeta_{x}}(x)\right)^{4}}{(\phi(x))^{5}}\right) \nabla \rho\left(\frac{z}{\phi(x)}\right) \cdot z \\
& \left.+\left(\frac{6\left(\phi_{, \zeta_{x}}(x)\right)^{2}}{(\phi(x))^{4}}-\frac{2 \phi_{, \zeta_{x} \zeta_{x}}(x)}{(\phi(x))^{3}}\right) \rho\left(\frac{z}{\phi(x)}\right)\right) d z .
\end{aligned}
$$

Note for $x \in N_{8 \epsilon}(\partial \Omega) \cap \Omega,\left|\phi_{, \zeta_{x}}(x)\right| \leq c$ and $\left|\phi_{\zeta_{x} \zeta_{x}}(x)\right| \leq c \epsilon^{-1} \leq c(\phi(x))^{-1}$. So applying (5.32) to (5.31)

$$
\begin{aligned}
& \left|\int u(x-z) \frac{\partial^{2}}{\partial \zeta_{x}^{2}}\left(\rho_{\phi(x)}(z)\right)\right| \\
& \leq \int\left|u(x-z)-l_{\zeta_{x}}(x-z)\right| \mid\left(\nabla^{2} \rho\left(\frac{z}{\phi(x)}\right): z \otimes z\right) \frac{\left(\phi, \zeta_{x}(x)\right)^{2}}{(\phi(x))^{6}} \\
& \quad+\left(\frac{-\phi_{, \zeta_{x} \zeta_{x}}(x)}{(\phi(x))^{4}}+\frac{6\left(\phi_{, \zeta_{x}}(x)\right)^{4}}{(\phi(x))^{5}}\right) \nabla \rho\left(\frac{z}{\phi(x)}\right) \cdot z \\
& \quad+\left(\frac{6\left(\phi_{, \zeta_{x}}(x)\right)^{2}}{(\phi(x))^{4}}-\frac{2 \phi_{, \zeta_{x} \zeta_{x}}(x)}{(\phi(x))^{3}}\right) \rho\left(\frac{z}{\phi(x)}\right) \mid d z \\
& \quad \leq c \int_{B_{\phi(x)}(0)} \frac{\left|u(x-z)-l_{\zeta_{x}}(x-z)\right|}{(\phi(x))^{4}} d z\left(\left\|\nabla^{2} \rho\right\|_{\infty}+\|\nabla \rho\|_{\infty}+\|\rho\|_{\infty}\right) \\
& \quad \leq c \int_{B_{\phi(x)}(x)}\left|u(z)-l_{\zeta_{x}}(z)\right|(\phi(x))^{-4} d z \\
& \quad \stackrel{(5.26)}{\leq} c \epsilon^{-\frac{1}{2}} .
\end{aligned}
$$

Define $h(x):=\int \rho_{\phi(x)}(z) d z$. Note that $h \equiv 1$ and so $\frac{\partial h}{\partial \zeta_{x}}(x)=\int \frac{\partial}{\partial \zeta_{x}}\left(\rho_{\phi(x)}(z)\right) d z=0$. 
So

$$
\begin{aligned}
& \left|\int u_{, \varsigma_{x}}(x-z) \frac{\partial}{\partial \varsigma_{x}}\left(\rho_{\phi(x)}(z)\right) d z\right| \\
& =\left|\int\left(u_{, \varsigma_{x}}(x-z)-1\right) \frac{\partial}{\partial \varsigma_{x}}\left(\rho_{\phi(x)}(z)\right) d z\right| \\
& \stackrel{\text { (5.9), (5.18) }}{\leq} c \epsilon^{-\frac{1}{2}} u(x) \mid \int \phi_{, \varsigma_{x}}(x)\left(\nabla \rho\left(\frac{z}{\phi(x)}\right) \cdot z(\phi(x))^{-4}\right. \\
& \left.+2 \rho\left(\frac{z}{\phi(x)}\right)(\phi(x))^{-3}\right) \\
& \leq c \epsilon^{-\frac{1}{2}} \text {. }
\end{aligned}
$$

Finally we estimate the first term from (5.14):

$$
\begin{aligned}
\left|\int u_{, \varsigma_{x} \varsigma_{x}}(x-z) \rho_{\phi(x)}(z) d z\right| & \leq\left\|\nabla^{2} u\right\|_{L^{\infty}\left(B_{4 \rho_{\phi}(x)}(x)\right)}\left|\int \rho_{\phi(x)}(z) d z\right| \\
& \stackrel{\text { (5.15) }}{\leq} c \epsilon^{-\frac{1}{2}} .
\end{aligned}
$$

Putting (5.33), (5.34) and (5.35) together and applying this to (5.14) we have

$$
\left|\psi_{, \zeta_{x} \varsigma_{x}}(x)\right| \leq c \epsilon^{-\frac{1}{2}} \text { for any } x \in N_{8 \epsilon}(\partial \Omega) \cap \Omega \text {. }
$$

Now by (5.12) for any $x \in N_{8 \epsilon}(\partial \Omega) \cap \Omega$ we have

$$
\begin{aligned}
&\left|\psi_{, \omega_{x} \zeta_{x}}(x)\right| \leq \int\left|\nabla^{2} u(x-z)\right| \rho_{\phi(x)}(z) d z \\
&+\int\left|u_{, \omega_{x}}(z-x) \frac{\partial}{\partial \varsigma_{x}}\left(\rho_{\phi(x)}(z)\right)\right| d z \\
& \stackrel{(5.18),(5.15)}{\leq} c \epsilon^{-\frac{1}{2}}+c \epsilon^{\frac{1}{2}} \int\left|\frac{\partial}{\partial \zeta_{x}}\left(\rho_{\phi(x)}(z)\right)\right| d z \\
& \stackrel{(5.9)}{\leq} c \epsilon^{-\frac{1}{2}} .
\end{aligned}
$$

And by (5.13)

$$
\begin{gathered}
\left|\psi_{\omega_{x} \omega_{x}(x) \mid} \leq\right| \int u_{, \omega_{x} \omega_{x}}(x-z) \rho_{\phi(x)}(z) d z \mid \\
\stackrel{(5.15)}{\leq} c \epsilon^{-\frac{1}{2}} .
\end{gathered}
$$

Putting (5.36), (5.37) and (5.38) together establishes (5.30).

Proof of Lemma completed. From Step 3 for any $x \in N_{8 \epsilon}(\partial \Omega) \cap \Omega$ we have

$$
\left.|| \nabla \psi(x)\right|^{2}-\left.1\right|^{2} \leq c \epsilon
$$

so (5.2) follows. In the same way from Step 4 (5.30) and (5.3) follows. 
Since for any $x \in \Omega \backslash N_{8 \epsilon}(\partial \Omega)$ we know $u(x) \geq \epsilon$ and so $\phi(x)=w(u(x))=\epsilon$ and thus $\rho_{\phi(x)}(z)=\rho\left(\frac{z}{\epsilon}\right) \epsilon^{-1}$ and there for $\psi(x)=\int u(x-z) \rho_{\epsilon}(z) d z$. Thus (5.4) is established. Finally by (5.21), (5.5) follows.

Lemma 5.4. Let $\Omega$ be a convex domain and $\left|\Omega \triangle B_{1}(0)\right| \leq \beta$. Let $u(x)=d(x, \partial \Omega)$ and for $\varepsilon>0$ define $u_{\varepsilon}:=u * \rho_{\varepsilon}$. For any $a \in \Omega \backslash N_{4 \varepsilon}(\partial \Omega)$ we have

$$
|| \nabla u_{\varepsilon}(x)|-1| \leq c \varepsilon^{-1} V\left(\nabla u, B_{4 \varepsilon}(a)\right) \text { for any } x \in B_{2 \varepsilon}(a) .
$$

Proof. Firstly recall that since $u$ is concave we have $\nabla u$ is BV. Let $w=f_{B_{4 \varepsilon}(a)} \nabla u d x$. By Poincaré's inequality (see Remark 3.45 [4])

$$
\int_{B_{4 \varepsilon}(a)}|\nabla u-w| d z \leq c \varepsilon V\left(\nabla u, B_{4 \varepsilon}(a)\right) .
$$

Now

$$
\begin{aligned}
\pi 16 \varepsilon^{2}|1-| w|| & =\int_{B_{4 \varepsilon}(a)}|1-| w|| d z \\
& =\int_{B_{4 \varepsilon}(a)}|| \nabla u|-| w|| d z \\
& \stackrel{(5.40)}{\leq} c \varepsilon V\left(\nabla u, B_{4 \varepsilon}(a)\right) .
\end{aligned}
$$

Thus $|1-| w|| \leq c \frac{V\left(\nabla u, B_{4 \varepsilon}(a)\right)}{\varepsilon}$ and so there must exists $v \in S^{1}$ such that $|v-w| \leq$ $|1-| w||$ hence putting this together with (5.40) we have

$$
f_{B_{4 \varepsilon}(a)}|\nabla u-v| d z \leq c \frac{V\left(\nabla u, B_{4 \varepsilon}(a)\right)}{\varepsilon} .
$$

Hence for any $w \in B_{2 \varepsilon}(a)$

$$
\begin{aligned}
\left|\nabla u_{\varepsilon}(w)-v\right| & =\left|\int(\nabla u(z)-v) \rho_{\varepsilon}(w-z) d z\right| \\
& \leq c \varepsilon^{-2}\left|\int(\nabla u(z)-v) \rho\left(\varepsilon^{-1}(z-w)\right) d z\right| \\
& \leq c \varepsilon^{-2} \int_{B_{2 \varepsilon}(w)}|\nabla u(z)-v| d z \\
& \stackrel{(5.41)}{\leq} c \frac{V\left(\nabla u, B_{4 \varepsilon}(a)\right)}{\varepsilon} .
\end{aligned}
$$

This completes the proof of Lemma 5.4. 
Lemma 5.5. Let $\Omega$ be a convex domain and $\left|\Omega \triangle_{1}(0)\right| \leq \beta$. Let $u(x)=d(x, \partial \Omega)$ and define $u_{\varepsilon}:=u * \rho_{\varepsilon}$. Define $\Lambda:=\Omega \backslash\left(N_{8 \varepsilon}(\partial \Omega) \cup B_{4 \beta^{\frac{1}{8}}}(0)\right)$, we will show that for any $\varepsilon \in\left(0, \frac{\beta^{\frac{1}{2}}}{4}\right]$

$$
\left.\left.\int_{\Lambda} \varepsilon^{-1}|1-| \nabla u_{\varepsilon}\right|^{2}\right|^{2}+\varepsilon\left|\nabla^{2} u_{\varepsilon}\right|^{2} d z \leq c \beta^{\frac{3}{16}}
$$

Proof of Lemma. By the 5r Covering Theorem [20, Theorem 2.1], we can find a finite collection of balls $J:=\left\{B_{\frac{2 \varepsilon}{5}}\left(x_{i}\right): i=1,2, \ldots m\right\}$ that are piecewise disjoint and $\Lambda \subset \bigcup_{i=1}^{m} B_{2 \varepsilon}\left(x_{i}\right)$.

Note that for any $i=1,2, \ldots n$, since the set of ball in $J$ are pairwise disjoint, for some constant $C_{1}$ there are at most $C_{1}$ balls from the set $\left\{B_{5 \varepsilon}\left(x_{k}\right): k=1, \ldots m\right\}$ intersecting $B_{5 \varepsilon}\left(x_{i}\right)$. Thus $\left\|\sum_{i=1}^{m} \mathbb{1}_{B_{5 \varepsilon}\left(x_{i}\right)}\right\|_{L^{\infty}(\Omega)} \leq C_{1}$ and this obviously implies $\left\|\sum_{i=1}^{m} \mathbb{1}_{B_{2 \varepsilon}\left(x_{i}\right)}\right\|_{L^{\infty}(\Omega)} \leq C_{1}$.

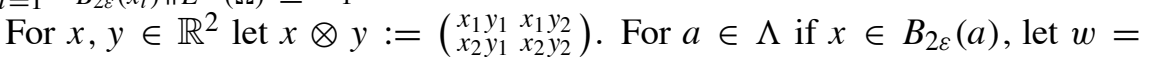
$f_{B_{\varepsilon}(x)} \nabla u d x$. Now

$$
\begin{aligned}
\left|\nabla^{2} u_{\varepsilon}(x)\right| & =\left|\int \nabla u(z) \otimes \nabla \rho_{\varepsilon}(x-z) d z\right| \\
& \leq\left|\int(\nabla u(z)-w) \otimes \nabla \rho\left(\frac{x-z}{\varepsilon}\right) \varepsilon^{-3} d z\right| \\
& \leq c \varepsilon^{-3}\left|\int_{B_{2 \varepsilon}(x)}(\nabla u-w) d z\right| \\
& \stackrel{(5.40)}{\leq} c \varepsilon^{-2} V\left(\nabla u, B_{4 \varepsilon}(a)\right) .
\end{aligned}
$$

So

$$
\begin{aligned}
\int_{\Lambda}\left|\nabla^{2} u_{\varepsilon}\right|^{2} d z & \leq \sum_{i=1}^{m} c \int_{B_{2 \varepsilon}\left(x_{i}\right)}\left|\nabla^{2} u_{\varepsilon}\right|^{2} d z \\
& \leq c \sum_{i=1}^{m} \varepsilon^{2}\left\|\nabla^{2} u_{\varepsilon}\right\|_{L^{\infty}\left(B_{2 \varepsilon}\left(x_{i}\right)\right)}^{2} \\
& \quad(5.43) \\
& \leq \varepsilon^{2}\left(\sum_{i=1}^{m} \varepsilon^{-4}\left(V\left(\nabla u, B_{4 \varepsilon}\left(x_{i}\right)\right)\right)^{2}\right) \\
& (4.11) \\
& \leq \beta^{\frac{3}{16}} \varepsilon^{-1}\left(\sum_{i=1}^{m} V\left(\nabla u, B_{4 \varepsilon}\left(x_{i}\right)\right)\right) \\
& \leq c \beta^{\frac{3}{16}} \varepsilon^{-1} V(\nabla u, \Lambda) \\
& (4.10) \\
& \leq c \varepsilon^{-1} \beta^{\frac{3}{16}} .
\end{aligned}
$$


Now

$$
\begin{aligned}
\left.\left.\int_{\Lambda}|1-| \nabla u_{\varepsilon}\right|^{2}\right|^{2} d z & \leq c \sum_{i=1}^{m} \int_{B_{2 \varepsilon}\left(x_{i}\right)}|1-| \nabla u_{\varepsilon}||^{2} d z \\
& \leq \sum_{i=1}^{(4.11),(5.39)} c \varepsilon^{2} \beta^{\frac{3}{16}}\left\||1-| \nabla u_{\varepsilon}||\right\|_{L^{\infty}\left(B_{2 \varepsilon}\left(x_{i}\right)\right)} \\
& \stackrel{(5.39)}{\leq} \sum_{i=1}^{m} c \varepsilon \beta^{\frac{3}{16}} V\left(\nabla u, B_{4 \varepsilon}\left(x_{i}\right)\right) \\
& \leq c \varepsilon \beta^{\frac{3}{16}} V\left(\nabla u, \Omega \backslash B_{2 \beta^{\frac{1}{8}}}(0)\right) \\
& (4.10) \\
& \leq \beta^{\frac{3}{16}} \varepsilon .
\end{aligned}
$$

Putting (5.45) together with (5.44) establishes (5.42).

Lemma 5.6. Let $\eta(x)=|x|, \varepsilon>0$ and define $\eta_{\varepsilon}(x):=\int \eta(z) \rho_{\varepsilon}(x-z) d z$. Then

$$
\left.\left.\int_{B_{1}(0)}|1-| \nabla \eta_{\varepsilon}\right|^{2}\right|^{2} d z \leq c \log \left(\varepsilon^{-1}\right) \varepsilon^{2}
$$

and

$$
\int_{B_{1}(0)}\left|\nabla^{2} \eta_{\varepsilon}\right|^{2} d z \leq c \log \left(\varepsilon^{-1}\right) .
$$

Proof of Lemma. Note for $x \notin B_{2 \varepsilon}(0), z \in B_{\varepsilon}(x)$

$$
\begin{aligned}
\left|\frac{z}{|z|}-\frac{x}{|x|}\right| & \leq\left|\frac{z|x|-x|z|}{|z||x|}\right| \\
& \leq\left|\frac{z|x|-x|x|}{|z||x|}\right|+\left|\frac{x|x|-x|z|}{|z||x|}\right| \\
& \leq \frac{c \varepsilon}{|x|-\varepsilon} .
\end{aligned}
$$

So for $x \notin B_{4 \varepsilon}(0)$

$$
\begin{aligned}
\left|\nabla \eta_{\varepsilon}(x)-\frac{x}{|x|}\right| & =\left|\int \rho_{\varepsilon}(x-z)\left(\frac{x}{|x|}-\frac{z}{|z|}\right) d z\right| \\
& \stackrel{(5.48)}{\leq} \frac{c \varepsilon}{|x|-\varepsilon} .
\end{aligned}
$$


Since $\int \frac{x}{|x|} \otimes \nabla \rho_{\varepsilon}(x-z) d z=0$, for any $x \notin B_{4 \varepsilon}(0)$

$$
\begin{aligned}
& \nabla^{2} \eta_{\varepsilon}(x)=\left|\int \nabla \eta_{\varepsilon}(z) \otimes \nabla \rho_{\varepsilon}(x-z) d z\right| \\
&=\left|\int\left(\nabla \eta_{\varepsilon}(z)-\frac{z}{|z|}\right) \otimes \nabla \rho_{\varepsilon}(x-z) d z\right| \\
&+\left|\int\left(\frac{x}{|x|}-\frac{z}{|z|}\right) \otimes \nabla \rho_{\varepsilon}(x-z) d z\right| \\
& \underset{(5.48),(5.49)}{\leq} \frac{c \varepsilon}{|x|-\varepsilon}\left|\int \nabla \rho_{\varepsilon}(x-z) d z\right| \\
& \leq \frac{c}{|x|-\varepsilon} .
\end{aligned}
$$

Hence

$$
\begin{aligned}
\int_{B_{1}(0) \backslash B_{4 \varepsilon}(0)}\left|\nabla^{2} \eta_{\varepsilon}(x)\right|^{2} d x & \stackrel{(5.50)}{=} c \int_{4 \varepsilon}^{1} \int_{\partial B_{h}(0)}\left(\frac{1}{|z|-\varepsilon}\right)^{2} d H^{1} z d r \\
& \leq c \int_{\varepsilon}^{1} \frac{1}{r} d r \\
& \leq c \log \left(\varepsilon^{-1}\right) .
\end{aligned}
$$

Now as $\left|\nabla \eta_{\epsilon}(x)\right| \leq c$ and $\left|\nabla^{2} \eta_{\epsilon}(x)\right| \leq c \epsilon^{-1}$ for any $x \in B_{1-\epsilon}(0)$ so

$$
\int_{B_{4 \epsilon}(0)}\left|\nabla^{2} \eta_{\epsilon}\right|^{2} d z \leq c \epsilon .
$$

Thus putting this together with (5.51) establishes (5.47).

Note ||$\nabla \eta_{\varepsilon}(x)|-1|^{2} \leq\left|\nabla \eta_{\varepsilon}(x)-\frac{x}{|x|}\right|^{2} \stackrel{(5.49)}{\leq} c \frac{\epsilon^{2}}{(|x|-\epsilon)^{2}}$ so arguing in the same way as in (5.51) we have (5.46).

\subsection{Proof of Proposition 5.1}

Let $u(x)=d(x, \partial \Omega)$, let $w: \mathbb{R}_{+} \rightarrow \mathbb{R}_{+}$be the smooth monotonic function from the proof of Lemma 5.3. So $w$ satisfies (5.6) and sup $|\ddot{w}| \leq c \epsilon^{-1}$. As in Lemma 5.3; for $x \in N_{\epsilon}(\partial \Omega) \cap \Omega$ define

$$
\phi(x)=w(u(x)) .
$$

Let

$$
v(x):=\min \left\{u(x), 1-8 \beta^{\frac{3}{32}}+|x|\right\}
$$

and define

$$
\xi(x)=\int v(x-z) \rho_{\phi(x)}(z) d z .
$$


Let $\Pi:=\left\{x: u(x)>1-8 \beta^{\frac{3}{32}}+|x|\right\}$. Define $\Lambda_{0}:=\Omega \backslash\left(N_{8 \epsilon}(\partial \Omega) \cup N_{\epsilon}(\Pi)\right)$. Note that $\xi(x)=u_{\epsilon}(x)$ for any $x \in \Lambda_{0}$.

Recall from (5.10) the function $\psi$ defined in Lemma 5.3. Note that for any $x \in N_{8 \epsilon}(\partial \Omega) \cap \Omega$ function $\phi$ we defined by (5.52) is identical to $\phi$ defined by (5.7) in Lemma 5.3. Hence as $u=v$ in $N_{8 \epsilon}(\partial \Omega) \cap \Omega$ we have $\xi(x)=\psi(x)$ for any $x \in N_{8 \epsilon}(\partial \Omega) \cap \Omega$ thus

$$
\left.\left.\int_{N_{8 \epsilon}(\partial \Omega) \cap \Omega} \epsilon^{-1}|1-| \nabla \xi\right|^{2}\right|^{2}+\epsilon\left|\nabla^{2} \xi\right|^{2} d x \stackrel{(5.2),(5.3)}{\leq} c \epsilon .
$$

Since $\psi=u_{\epsilon}$ in $\Lambda_{0}$, from (5.42) we have $\left.\left.\int_{\Lambda_{0}} \epsilon^{-1}|1-| \nabla \xi\right|^{2}\right|^{2}+\epsilon\left|\nabla^{2} \xi\right|^{2} d x \leq$ $c \beta^{\frac{3}{16}}$ and so putting this two inequalities together we have

$$
\left.\left.\int_{\Omega \backslash N_{\epsilon}(\Pi)} \epsilon^{-1}|1-| \nabla \xi\right|^{2}\right|^{2}+\epsilon\left|\nabla^{2} \xi\right|^{2} d x \leq c \beta^{\frac{3}{16}} .
$$

Now, as for any $x \in \Pi \backslash N_{\epsilon}(\partial \Pi), w(x)=1-8 \beta^{\frac{3}{32}}+|x|$ and so $u_{\epsilon}(x)=\eta_{\epsilon}(x)+$ $\left(1-8 \beta^{\frac{3}{32}}\right)$ where $\eta(x)=|x|$ and $\eta_{\epsilon}=\eta * \rho_{\epsilon}$. So $\nabla \xi(x)=\nabla \eta_{\epsilon}(x)$ and $\nabla^{2} \xi(x)=$ $\nabla^{2} \eta_{\epsilon}(x)$ thus applying Lemma 5.6 we have

$$
\left.\left.\int_{\Pi \backslash N_{\epsilon}(\partial \Pi)} \epsilon^{-1}|1-| \nabla \xi\right|^{2}\right|^{2}+\epsilon\left|\nabla^{2} \xi\right|^{2} d x \stackrel{(5.46),(5.47)}{\leq} c \epsilon \log \left(\epsilon^{-1}\right) .
$$

Since $w$ is Lipschitz, $\xi$ is Lipschitz and so from (4.43) we have

$$
\left.\left.\int_{N_{\epsilon}(\partial \Pi)} \epsilon^{-1}|1-| \nabla \xi\right|^{2}\right|^{2} d x \leq c \beta^{\frac{3}{32}} .
$$

And note for any $x \in \Omega \backslash N_{\epsilon}(\partial \Omega)$

$$
\left|\nabla^{2} \xi(x)\right|=\epsilon^{-3}\left|\int \nabla v(z) \cdot \nabla \rho\left(\frac{x-z}{\epsilon}\right) d z\right| \leq c \epsilon^{-1}
$$

so

$$
\begin{gathered}
\int_{N_{\epsilon}(\partial \Pi)} \epsilon\left|\nabla^{2} \xi\right|^{2} d x \leq c \epsilon^{-1}\left|N_{\epsilon}(\partial \Pi)\right| \\
\stackrel{(4.43)}{\leq} c \beta^{\frac{3}{32}} .
\end{gathered}
$$

Putting these inequalities together we have

$$
\left.\left.\int_{N_{\epsilon}(\partial \Pi)} \epsilon^{-1}|1-| \nabla \xi\right|^{2}\right|^{2}+\epsilon\left|\nabla^{2} \xi\right|^{2} d x \leq c \beta^{\frac{3}{32}} .
$$

Now inequalities (5.53), (5.54) and (5.55) give us that $\xi$ satisfies (5.1). And since $\xi(x)=\psi(x)$ on $N_{\epsilon}(\partial \Omega) \cap \Omega$ from (5.5) satisfies $\nabla \xi(x) \cdot \eta_{x}=1$ for any $x \in \partial \Omega$. This completes the proof of Proposition 5.1. 


\subsection{Proof Corollary 1.2}

Let $\alpha=\inf _{y \in \Omega}\left|\Omega \triangle B_{1}(y)\right|$. Let $\beta=4(\alpha+\epsilon)$, note that since we can assume without loss of generality that $\alpha+\epsilon \leq \frac{1}{4}$ so $\beta \leq 1$. This implies $\beta \leq \beta^{\frac{1}{2}}$ and so $\epsilon \leq \frac{\beta^{\frac{1}{2}}}{4}$. Now we can also assume without loss of generality that $\left|\Omega \triangle B_{1}(0)\right| \leq \beta$. So we can apply Proposition 5.1 which gives us the existence of $\xi \in \Lambda(\Omega)$ such that(5.1) hold true. Hence we have that $\inf _{u \in \Lambda(\Omega)} I_{\epsilon}(u) \leq c \beta^{\frac{3}{32}}$. Let $v \in \Lambda(\Omega)$ be the minimiser of $I_{\epsilon}$ and since $v$ satisfies

$$
\left.\int_{\Omega}|1-| \nabla v\right|^{2}|| \nabla^{2} v\left|d z \leq \int_{\Omega} \epsilon^{-1}\right| 1-\left.|\nabla v|^{2}\right|^{2}+\epsilon\left|\nabla^{2} v\right|^{2} d z \leq c \beta^{\frac{3}{32}}
$$

and as $\epsilon \in\left(0, \frac{\beta^{\frac{1}{2}}}{4}\right)$

$$
\left.\left.\int_{\Omega}|1-| \nabla v\right|^{2}\right|^{2} d z \leq c \beta^{\frac{19}{32}} .
$$

Thus we have that (1.4), (1.5) are satisfied and hence by Theorem 1.4

$$
\int_{\Omega}\left|\nabla v(z)+\frac{z}{|z|}\right|^{2} d z \leq c \beta^{\frac{1}{5462}} .
$$

Applying Lemma 4.4 we have $\int_{\Omega \backslash B}{ }_{\beta^{\frac{1}{8}}}(0)|\nabla v-\nabla \zeta|^{2} \leq c \beta^{\frac{1}{5462}}$. So arguing in the same way as the proof of Corollary 1.3 we have $\|v-\zeta\|_{W^{1,2}(\Omega)} \leq c \beta^{\frac{1}{5462}} \leq c(\epsilon+$ $\alpha)^{\frac{1}{5462}}$.

\section{References}

[1] F. Alouges, T. RiviÈre and S. SERFATY, Nèel and cross-tie wall energies for planar micromagnetic configurations, a tribute to J. L. Lions, ESAIM Control Optim. Calc. Var. 8 (2002), 31-68.

[2] L. Ambrosio, C. De Lellis and C. Mantegazza, Line energies for gradient vector fields in the plane, Calc. Var. Partial Differential Equations 9 (1999), 327-255.

[3] L. Ambrosio and C. DE Lellis, A note on admissible solutions of $1 D$ scalar conservation laws and 2D Hamilton-Jacobi equations, J. Hyperbolic Differ. Equ. 1 (2004), 813-826.

[4] L. Ambrosio, N. Fusco and D. Pallara, "Functions of Bounded Variation and Free Discontinuity Problems", Oxford Mathematical Monographs, The Clarendon Press, Oxford University Press, New York, 2000.

[5] L. Ambrosio, M. LeCUMBerRy and T. Rivière, A viscosity property of minimizing micromagnetic configurations, Comm. Pure Appl. Math. 56 (2003), 681-688

[6] L. Ambrosio, B. KirchHeim, M. Lecumberry and T. Rivière, On the rectifiability of defect measures arising in a micromagnetics model, In: "Nonlinear Problems in Mathematical Physics and Related Topics", II, Int. Math. Ser. (N.Y.), 2, Kluwer/Plenum, New York, 2002, 29-60. 
[7] P. Aviles and Y. GigA, A mathematical problem related to the physical theory of liquid crystal configurations, In: "Miniconference on Geometry and Partial Differential Equations", Proc. Centre Math. Anal. Austral. Nat. Univ., 12, Austral. Nat. Univ., Canberra, $1987,1-16$.

[8] S. CONTI and C. DE LELLIS, Sharp upper bounds for a variational problem with singular perturbation, Math. Ann. 338 (2007), 119-146.

[9] C. DE LELLIS and F. OTTO, Structure of entropy solutions to the eikonal equation, J. Eur. Math. Soc. (JEMS) 5 (2003), 107-145.

[10] C. De Lellis, F. OtTo and M. Westdickenberg, Structure of entropy solutions for multi-dimensional scalar conservation laws, Arch. Ration. Mech. Anal. 170 (2003), 137184.

[11] A. DeSimone, S. MÜLler, R. KoHn and F. OTto, A compactness result in the gradient theory of phase transitions, Proc. Roy. Soc. Edinburgh Sect. A 131 (2001), 833-844.

[12] R. DiPerna, P. LiOns and Y. MeYer, $L^{p}$ regularity of velocity averages, Ann. Inst. $\mathrm{H}$. Poincaré Anal. Non Linéaire 8 (1991), 271-287.

[13] L. C. Evans and R. F. Gariepy, "Measure Theory and Fine Properties of Functions", Studies in Advanced Mathematics, CRC Press, Boca Raton, FL, 1992.

[14] G. Gioia and M. ORTIZ, Delamination of compressed thin films, Adv. Appl. Mech. 33 (1997), 119-192.

[15] P. JABIN and B. Perthame, Compactness in Ginzburg-Landau energy by kinetic averaging, Comm. Pure Appl. Math. 54 (2001), 1096-1109.

[16] P. Jabin, F. OtTo and B. Perthame, Line-energy Ginzburg-Landau models: zero-energy states, Ann. Sc. Norm. Super. Pisa Cl. Sci. (5) 1 (2002), 187-202.

[17] W. JIN and R. V. KoHN, Singular perturbation and the energy of folds, J. Nonlinear Sci. 10 (2000), 355-390.

[18] M. LECUMBERRY and T. RIVIÈRE, Regularity for micromagnetic configurations having zero jump energy, Calc. Var. Partial Differential Equations 15 (2002), 389-402.

[19] A. LORENT, A Poincaré type inequality for Aviles Giga energy and applications, in preparation.

[20] P. MatTila, "Geometry of Sets and Measures in Euclidean Spaces. Fractals and Rectifiability", Cambridge Studies in Advanced Mathematics, Vol. 44, Cambridge University Press, Cambridge, 1995.

[21] L. Modica and S. Mortola, Un esempio di $\Gamma$-convergenza, Boll. Un. Mat. Ital. B (5) 14 (1977), 285-299.

[22] F. MURAT, Compacite par compensation: condition necessaire et suffisante de continuite faible sous une hypothese de rang constant, Ann. Scuola Norm. Sup. Pisa Cl. Sci. (4) 8 (1981), 69-102.

[23] A. Poliakovsky, Upper bounds for singular perturbation problems involving gradient fields, J. Eur. Math. Soc. (JEMS) 9 (2007), 1-43.

[24] T. RIVIÈRE and S. SERFATY, Limiting domain wall energy for a problem related to micromagnetics, Comm. Pure Appl. Math. 54 (2001), 294-338.

[25] L. TARTAR, Compensated compactness and applications to partial differential equations, In: "Nonlinear analysis and mechanics: Heriot-Watt Symposium", Vol. IV, Res. Notes in Math., 39, Pitman, Boston, Mass.-London, 1979, 136-212.

Mathematics Department University of Cincinnati 2600 Clifton Ave. Cincinnati Ohio 45221, USA andrew.lorent@uc.edu 